\title{
Transformação dq Não Senoidal para Máquinas Síncronas com Ímã Permanente no Rotor
}

\author{
José Roberto B. de A. Monteiro
}

Tese apresentada à Escola de Engenharia de São Carlos, da Universidade de São Paulo, como parte dos requisitos para obtenção do Título de Doutor em Engenharia Elétrica

Orientador: Prof. Dr. Azauri Albano de Oliveira Jr. 
Primeiramente, gostaria de agradecer ao Prof. Dr. Azauri Albano de Oliveira Jr., pela excelente orientação. Ao Prof. Dr. Diógenes Pereira Gonzaga, pelas inúmeras contribuições sobre máquinas elétricas. Ao Prof. Dr. Manoel Luiz de Aguiar, pelas contribuições na área de controle vetorial.

Aos engenheiros mecânicos Pedro Zorzenon Neto e Carlos Eduardo Milhor e ao Prof. Dr. Luiz Carlos Passarini, pelas contribuições na área de engenharia mecânica, necessários paras as medições realizadas no trabalho.

Ao projetista mecânico José António Otoboni, pelo desenho e montagem das peças do sistema mecânico.

Ao pessoal do LACEP pelo apoio técnico e interesse na realização desse projeto.

Ao pessoal do Departamento de Engenharia Elétrica da Escola de Engenharia de São Carlos - Universidade de São Paulo.

À Free Software Foundation, que vem promovendo o desenvolvendo de Software livre, tornando possível o acesso a ferramentas de software sofisticadas por inúmeros usuários, sem as quais esse projeto não seria viável.

E por fim, à FAPESP - Fundação de Amparo à Pesquisa do Estado de São Paulo - pelo financiamento prestado a esse projeto de pesquisa, sem o qual esse projeto não seria possível. 


\section{Sumário}

Lista de Figuras $\quad$ v

$\begin{array}{ll}\text { Lista de Abreviaturas e Siglas } & \text { ix }\end{array}$

Lista de Símbolos $\quad$ xi

Resumo xvi

Abstract $\quad$ xvii

I Introdução 1

1 O Motor Síncrono com Ímã Permanente 2

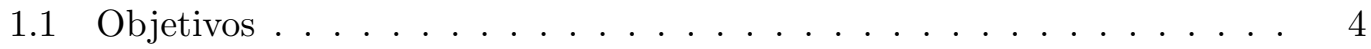

1.2 Divisão dos Capítulos . . . . . . . . . . . . . . . 4

2 Ondulações no Torque Eletromagnético $\quad 6$

2.1 Técnicas Utilizadas para Redução das Ondulações . . . . . . . . . . . 6

2.2 Particularidades do Método Proposto . . . . . . . . . . . . . . 12

$\begin{array}{lr}\text { II Desenvolvimento } & 14\end{array}$

3 Modelagem Matemática $\quad 15$

3.1 Modelagem Convencional por Fase . . . . . . . . . . . . . . . 15

3.2 Transformação $d q$ Não Senoidal $\ldots$. . . . . . . . . . . . . . . . . . 17

3.3 Modelo Vetorial da MSIP . . . . . . . . . . . . . . . . . . . . 19

3.3 .1 Particularização para o caso senoidal . . . . . . . . . . . . 23

3.3 .2 Perdas no cobre . . . . . . . . . . . . . . . . 23

3.4 Análise da MSIP em Regime Permanente . . . . . . . . . . . . . . . 24 
4 Simulação da MSIP $\quad 26$

4.1 Parâmetros da Máquina . . . . . . . . . . . . . . . . . . . 26

4.2 Simulação em Malha Fechada de Corrente . . . . . . . . . . . . . 30

4.3 Simulação em Malha Aberta de Corrente . . . . . . . . . . . . . . . . . 38

4.4 Simulação no Modo Seis-Pulsos . . . . . . . . . . . . . . . . . . . 41

5 Implementação do Controle Vetorial $\quad 44$

5.1 Implementação em Malha Aberta de Corrente . . . . . . . . . . . . . . 44

5.2 Medida Indireta de Torque . . . . . . . . . . . . . . . . . . . . 53

$\begin{array}{llr}\text { III Conclusões } & 58\end{array}$

6 Conclusões $\quad 59$

$\begin{array}{lll}\text { IV Apêndices } & 61\end{array}$

A Técnicas de Projeto de MSIPs

$\begin{array}{ll}\text { B Modelagem Convencional por Fase } & 64\end{array}$

$\begin{array}{ll}\text { C Transformações e Modelos Vetoriais } & 68\end{array}$

C.1 Transformações de Coordenadas . . . . . . . . . . . . . . . 68

C.2 Aplicação das Transformações de Coordenadas . . . . . . . . . . . . . . 69

C.2.1 Aplicação da Transformação $\alpha \beta 0$. . . . . . . . . . . . . . . 70

C.2.2 Aplicação da Transformação $d q$ com Ângulo dos Eixos $d q$ Igual ao Ângulo do Rotor da Máquina . . . . . . . . . . . . . . . . 71

C.2.3 Aplicação da Transformação $d q$ com Ângulo dos Eixos $d q$ Qualquer 72

$\begin{array}{ll}\text { D Dedução das equações } & \mathbf{7 4}\end{array}$

D.1 Dedução das equações do capítulo $3 \ldots \ldots$. . . . . . . . . 74

D.2 Dedução das equações apresentadas no apêndice C . . . . . . . . . . . . 80

$\begin{array}{lr}\text { E Descrição Física da Máquina } & 90\end{array}$

$\begin{array}{lll}\text { F Implementação do sistema de controle } & 94\end{array}$

F.1 Camada de Potência . . . . . . . . . . . . . . . . . . . . . . . . . . . . . 94

F.2 Camada de Interface Analógica/Digital . . . . . . . . . . . . . . . 96

F.3 Camada de Processamento Digital . . . . . . . . . . . . . . . . . . . 98 
G Descrição do Programa

101

V Referências

Referências Bibliográficas

106 


\section{Lista de Figuras}

FIGURA 3.1 Esquema físico simplificado do servo-motor (motor com 2 pólos). 16

FIGURA 3.2 Representação gráfica dos três eixos de coordenadas utilizados nas transformações aplicadas à MSIP. . . . . . . . . . . . . . . . . . 19

FIGURA 3.3 Diagrama em blocos da transformação $d q . \quad \ldots \ldots . \quad \ldots \ldots$

FIGURA 3.4 Diagrama em blocos da transformação $d q$ não senoidal completa. 19

FIGURA 3.5 Diagrama em blocos da transformação para as grandezas nos eixos $d q_{x}$ para os eixos $d q \ldots \ldots \ldots \ldots \ldots$

FIGURA 4.1 Tensão de linha da máquina, geradas pela variação do fluxo magnético concatenado pelas espiras do estator, produzido exclusivamente pelos ímãs do rotor. . . . . . . . . . . . . . . . . . . . . . 27

FIGURA 4.2 Forma de onda de tensão induzida da máquina, $F_{r a}, F_{r b}$ e $F_{r c}$, com amplitude unitária. . . . . . . . . . . . . . . . . . . 28

FIGURA 4.3 Parâmetro $a_{x}$ para a máquina utilizada, em função da posição

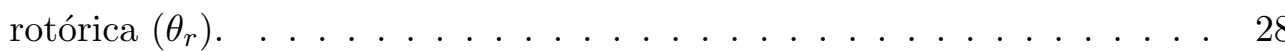

FIGURA 4.4 Parâmetro $\theta_{x}$ para a máquina utilizada, em função da posição

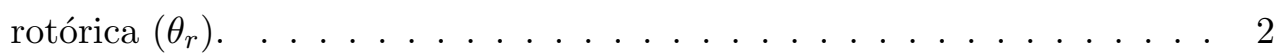

FIGURA 4.5 Parâmetro $\frac{d a_{x}}{d \theta_{r}}$ para a máquina utilizada, em função da posição

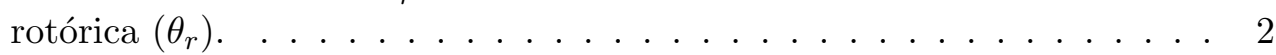

FIGURA 4.6 Parâmetro $\frac{d \theta_{x}}{d \theta_{r}}$ para a máquina utilizada, em função da posição

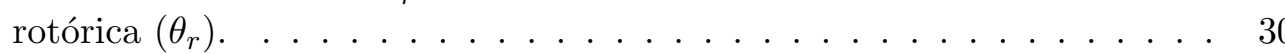


FIGURA 4.7 Diagrama de controle utilizado na simulação da máquina em malha fechada de corrente. . . . . . . . . . . . . . . . . . . . 32

FIGURA 4.8 Diagrama de controle utilizado na simulação da máquina em malha fechada de corrente.

FIGURA 4.9 Torque de partida e corrente da máquina acionada com controle vetorial (sem utilização de PWM).

FIGURA 4.10 Torque de partida e corrente da máquina acionada com controle vetorial, com $f_{P W M}=5,88 \mathrm{kHz}$.

FIGURA 4.11 Torque de partida e corrente da máquina acionada com controle vetorial, com $f_{P W M}=20 \mathrm{kHz}$.

FIGURA 4.12 MSIP seguindo referência de torque. . . . . . . . . . . . . . 36

FIGURA 4.13 MSIP seguindo referência de velocidade. . . . . . . . . . . . 37

FIGURA 4.14 MSIP seguindo referência de velocidade - detalhe do transitório inicial. . . . . . . . . . . . . . . . . . . . . . 38

FIGURA 4.15 MSIP seguindo referência de posição. . . . . . . . . . . . . . . 39

FIGURA 4.16 Esquema da simulação em malha aberta de corrente. . . . . . 39

FIGURA 4.17 Simulação em malha aberta de corrente, sem a utilização de PWM nas tensões aplicadas ao estator. . . . . . . . . . . . . . . . . . 40

FIGURA 4.18 Simulação em malha aberta de corrente, com $f_{P W M}=5,88$ $\mathrm{kHz}$

FIGURA 4.19 Simulação em malha aberta de corrente, com $f_{P W M}=20 \mathrm{kHz} . \quad 41$

FIGURA 4.20 Torque de partida e corrente da máquina acionada com inversor "seis-pulsos" $\left(f_{P W M}=5,88 \mathrm{kHz}\right) \ldots \ldots \ldots \ldots \ldots \ldots$

FIGURA 4.21 Torque de partida e corrente da máquina acionada com inversor "seis-pulsos" $\left(f_{P W M}=20 \mathrm{kHz}\right) . \ldots \ldots \ldots \ldots$

FIGURA 5.1 Simulação da corrente de fase e do torque eletromagnético (gráfico - a) e medida da corrente de fase (gráfico - b) na MSIP com controle vetorial em malha aberta de corrente (corrente: 2,13 A/div, $\left.\omega_{m}=24,6 \mathrm{rad} / \mathrm{s}\right) \ldots \ldots \ldots \ldots \ldots \ldots \ldots$ 
FIGURA 5.2 Simulação da corrente de fase e do torque eletromagnético (gráfico - a) e medida da corrente de fase (gráfico - b) na MSIP com controle vetorial em malha aberta de corrente (corrente: 1,06 A/div, $\left.\omega_{m}=67,6 \mathrm{rad} / \mathrm{s}\right) \ldots \ldots \ldots \ldots \ldots \ldots \ldots$

FIGURA 5.3 Simulação da corrente de fase e do torque eletromagnético (gráfico - a) e medida da corrente de fase (gráfico - b) na MSIP com controle vetorial em malha aberta de corrente (corrente: 2,13 A/div, $\left.\omega_{m}=59,8 \mathrm{rad} / \mathrm{s}\right) \ldots \ldots \ldots \ldots \ldots \ldots \ldots$

FIGURA 5.4 Simulação da corrente de fase e do torque eletromagnético (gráfico - a) e medida da corrente de fase (gráfico - b) na MSIP com controle vetorial em malha aberta de corrente (corrente: 2,13 A/div, $\left.\omega_{m}=11,3 \mathrm{rad} / \mathrm{s}\right) \ldots \ldots \ldots \ldots \ldots \ldots$

FIGURA 5.5 Simulação da corrente de fase e do torque eletromagnético (gráfico - a) e medida da corrente de fase (gráfico - b) na MSIP com controle vetorial em malha aberta de corrente (corrente: 1,06 A/div, $\left.\omega_{m}=22,5 \mathrm{rad} / \mathrm{s}\right) \ldots \ldots \ldots \ldots \ldots \ldots$

FIGURA 5.6 Simulação da corrente de fase e do torque eletromagnético (gráfico - a) e medida da corrente de fase (gráfico - b) na MSIP com controle vetorial em malha aberta de corrente (corrente: 1,06 A/div, $\left.\omega_{m}=44,8 \mathrm{rad} / \mathrm{s}\right)$.

FIGURA 5.7 Simulação da corrente de fase e do torque eletromagnético (gráfico - a) e medida da corrente de fase (gráfico - b) na MSIP com controle vetorial em malha aberta de corrente (corrente: 0,426 A/div, $\left.\omega_{m}=30,4 \mathrm{rad} / \mathrm{s}\right)$.

FIGURA 5.8 Sistema utilizado para medida da posição do rotor em função do tipo de controle utilizado.

FIGURA 5.9 Posição rotórica da máquina ao levantar carga através de roldana, com conversor no modo "seis-pulsos". As subfiguras (b) e (c) correspondem a ampliações da subfigura (a) 
FIGURA 5.10 Posição rotórica da máquina ao levantar carga através de roldana, com conversor utilizando controle vetorial em malha aberta de corrente. As subfiguras (b) e (c) correspondem a ampliações da subfi-

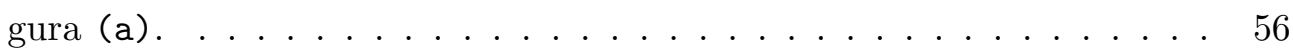

FIGURA B.1 Esquema físico simplificado do servo-motor (motor com 2 pólos). 65

FIGURA E.1 Corte transversal da máquina síncrona Siemens 1FT5. . . . . 91

FIGURA E.2 Perfil da máquina 1FT5, com o detalhe do conector de sinais. 92

FIGURA E.3 Conector do motor brushless 1FT5 062 - vista traseira do conector fêmea. . . . . . . . . . . . . . . . . . . . . . . . 93

FIGURA E.4 Sinais dos sensores de posição internos de efeito hall. . . . . . 93

FIGURA F.1 Sistema de controle proposto. . . . . . . . . . . . . 95

FIGURA F.2 Diagrama esquemático do módulo retificador. . . . . . . . . 95

FIGURA F.3 Diagrama esquemático do módulo inversor. . . . . . . . . . . . 96

FIGURA F.4 Diagrama em blocos da placa de interface analógica/digital. . 98

FIGURA F.5 Placa do processador. . . . . . . . . . . . . . . . . 100

FIGURA G.1 Algoritmo do programa de controle em malha aberta de corrente.102 


\title{
Lista de Abreviaturas e Siglas
}

\author{
A/D: analog to digital - analógico para digital; \\ DC: direct current - corrente contínua; \\ CA: Corrente alternada; \\ CC: Corrente contínua;
}

EESC: Escola de Engenharia de São Carlos;

FCEM: Força eletromotriz;

IGBT: insulated gate bipolar transistor - transistor bipolar de porta isolada;

LACEP: Laboratório de Controle e Eletrônica de Potência - SEL - EESC - USP;

MLP: Modulação em largura de pulso;

MSIP: máquina síncrona com ímã permanente no rotor e/ou máquina síncrona com ímã permanente na superfície do rotor;

OLVPWM: open loop vectorial pulse width modulation - modulação em largura de pulso vetorial em malha aberta;

PM: permanent magnet (ímã permanente);

PMSM: permanent magnet synchronous machine (máquina síncrona com ímã permanente);

PTC: positive thermal coefficient - resistor com coeficiente térmico positivo;

PWM: Pulse width modulation - modulação em largura de pulso;

RAM: random access memory - memória de acesso aleatório;

ROM: read-only memory - memória de apenas leitura;

SEL: Departamento de Engenharia Elétrica; 
SSPWM: six-step pulse width modulation - modulação em largura de pulso para inversor no modo "seis pulsos";

UART: universal assynchronous receiver/transmiter - receptor/transmissor assíncrono universal;

USP: Universidade de São Paulo. 


\section{Lista de Símbolos}

$a_{x}$ : amplitude do coeficiente da transformação $d q$ não senoidal;

$B$ : densidade de fluxo de entreferro devida ao rotor $(\mathrm{T})$;

$B_{c}$ : constante de atrito viscoso da carga $\left(\mathrm{kg} \cdot \mathrm{m}^{2} / \mathrm{s}\right) ;$

$B_{m}$ : constante de atrito viscoso nos mancais do motor $\left(\mathrm{kg} \cdot \mathrm{m}^{2} / \mathrm{s}\right)$;

$c_{x}$ : coeficiente complexo da transformação $d q$ não senoidal;

$e_{a}:$ tensão induzida na fase a $(\mathrm{V})$;

$e_{b}$ : tensão induzida na fase $\mathrm{b}(\mathrm{V})$;

$e_{c}$ : tensão induzida na fase c $(\mathrm{V})$;

$f_{P W M}$ : freqüência de $\mathrm{PWM}(\mathrm{Hz})$;

$F_{r a}$ : forma de onda de tensão induzida na fase a do estator, devido somente ao campo do rotor;

$F_{r b}$ : forma de onda de tensão induzida na fase b do estator, devido somente ao campo do rotor;

$F_{r c}$ : forma de onda de tensão induzida na fase c do estator, devido somente ao campo do rotor;

$F_{r \alpha}$ : forma de onda de tensão induzida projetada no eixo $\alpha$;

$F_{r \beta}$ : forma de onda de tensão induzida projetada no eixo $\beta$;

$F_{r \alpha \beta}$ : forma de onda de tensão induzida projetada nos eixos $\alpha \beta$;

$F_{r d}$ : forma de onda de tensão induzida projetada no eixo $d$;

$F_{r q}$ : forma de onda de tensão induzida projetada no eixo $q$;

$F_{r d q}:$ forma de onda de tensão induzida projetada nos eixos $d q$; 
$F_{r d_{x}}$ : forma de onda de tensão induzida projetada no eixo $d_{x}$;

$F_{r q_{x}}$ : forma de onda de tensão induzida projetada no eixo $q_{x}$;

$F_{r d q_{x}}$ : forma de onda de tensão induzida projetada nos eixos $d q_{x}$;

$G_{d}, G_{q}, G_{i q x}$, e $G_{v d x}$ : blocos de controle do controle vetorial em malha fechada de corrente;

$i_{0}$ : corrente de estator, componente de fase $0(\mathrm{~A})$;

$i_{a}$ : corrente de estator na fase a $(\mathrm{A}) ;$

$i_{b}$ : corrente de estator na fase $\mathrm{b}(\mathrm{A})$;

$i_{c}$ : corrente de estator na fase c $(\mathrm{A})$;

$i_{d}$ : corrente de estator projetada no eixo $d(\mathrm{~A})$;

$i_{q}$ : corrente de estator projetada no eixo $q(\mathrm{~A})$;

$i_{d q}:$ corrente de estator projetada nos eixos $d q(\mathrm{~A})$

$i_{d_{x}}$ : corrente de estator projetada no eixo $d_{x}(\mathrm{~A}) ;$

$i_{q_{x}}$ : corrente de estator projetada no eixo $q_{x}(\mathrm{~A}) ;$

$i_{d q_{x}}:$ corrente de estator projetada nos eixos $d q_{x}(\mathrm{~A})$;

$i_{d_{x} r e f}$ : corrente de referência para o eixo $d_{x}(\mathrm{~A}) ;$

$i_{q_{x} r e f}$ : corrente de referência para o eixo $q_{x}(\mathrm{~A})$;

$i_{\alpha}$ : corrente de estator projetada no eixo $\alpha(\mathrm{A})$;

$i_{\beta}$ : corrente de estator projetada no eixo $\beta(\mathrm{A})$;

$i_{\alpha \beta}$ : corrente de estator projetada nos eixos $\alpha \beta$ na forma complexa (A);

$i_{k_{i x}}:$ índice para $k_{i_{x}}$

$i_{\delta_{a}}$ : valor discretizado do ciclo de trabalho da fase a;

$i_{\delta_{b}}$ : valor discretizado do ciclo de trabalho da fase $\mathrm{b} ;$

$i_{\delta_{c}}$ : valor discretizado do ciclo de trabalho da fase c;

$J_{c}$ : momento de inércia da carga aplicada ao rotor $\left(\mathrm{kg} \cdot \mathrm{m}^{2}\right)$;

$J_{m}$ : momento de inércia do rotor da máquina $\left(\mathrm{kg} \cdot \mathrm{m}^{2}\right)$;

$k_{e}$ : constante elétrica do motor $(\mathrm{V} \cdot \mathrm{s} / \mathrm{rad})$;

$k_{i x}$ : relação entre $i_{d_{x}}$ e $i_{q_{x}}$; 
$L$ : matriz de indutâncias da máquina $(\mathrm{H})$;

$L_{s}$ : auto-indutância de uma fase do estator $(\mathrm{H})$;

$M:$ índice de modulação;

$M_{v}$ : índice de modulação calculado para $v_{d q_{x}}$;

$M_{s}$ : indutância mútua entre duas fases do estator $(\mathrm{H})$;

$P_{m}:$ potência útil no eixo da máquina $(\mathrm{W})$;

$R_{s}$ : resistência total de uma fase do estator $(\Omega)$;

$T$ : matriz da transformada de Concordia;

$T_{c}$ : torque de carga aplicado ao eixo da máquina $(\mathrm{N} \cdot \mathrm{m}) ;$

$T_{e l}$ : torque eletromagnético produzido no eixo da máquina $(\mathrm{N} \cdot \mathrm{m}) ;$

$T_{\text {elref }}$ : torque eletromagnético de referência para o eixo da máquina $(\mathrm{N} \cdot \mathrm{m})$;

$T_{\text {elmax }}:$ torque eletromagnético máximo desenvolvido pela máquina $(\mathrm{N} \cdot \mathrm{m})$;

$V_{B U S}$ : valor de tensão aplicada ao barramento DC da ponte inversora (V);

$v_{0}$ : tensão de estator, componente de fase $0(\mathrm{~V})$;

$v_{a}$ : tensão aplicada ao terminal da fase a $(\mathrm{V})$;

$v_{b}$ : tensão aplicada ao terminal da fase $\mathrm{b}(\mathrm{V})$;

$v_{c}$ : tensão aplicada ao terminal da fase c $(\mathrm{V})$;

$v_{n}$ : tensão no terminal central da máquina $(\mathrm{V})$;

$v_{a n}$ : tensão aplicada na fase a do estator, em relação ao neutro $(\mathrm{V})$;

$v_{b n}$ : tensão aplicada na fase $\mathrm{b}$ do estator, em relação ao neutro $(\mathrm{V})$;

$v_{c n}$ : tensão aplicada na fase c do estator, em relação ao neutro $(\mathrm{V})$;

$v_{\alpha}$ : tensão de estator projetada no eixo $\alpha(\mathrm{V})$;

$v_{\beta}$ : tensão de estator projetada no eixo $\beta(\mathrm{V})$;

$v_{\alpha \beta}$ : tensão projetada nos eixos $\alpha \beta$ na forma complexa $(\mathrm{V})$;

$v_{d}$ : tensão de estator projetada no eixo $d(\mathrm{~V})$;

$v_{q}$ : tensão de estator projetada no eixo $q(\mathrm{~V})$;

$v_{d q}$ : tensão de estator projetada nos eixos $d q$ na forma complexa $(\mathrm{V})$; 
$v_{d_{x}}:$ tensão de estator projetada no eixo $d_{x}(\mathrm{~V})$;

$v_{q_{x}}:$ tensão de estator projetada no eixo $q_{x}(\mathrm{~V})$;

$v_{d q_{x}}$ : tensão de estator projetada nos eixos $d q_{x}$ na forma complexa $(\mathrm{V})$;

$V_{v}$ : módulo de $v_{d q_{x}}$;

$z_{p}$ : número de pares de pólos do motor;

$\gamma$ : valor de ângulo utilizado nas expressões para o PWM descontínuo (rad);

$\delta_{a}:$ ciclo de trabalho da fase a;

$\delta_{b}$ : ciclo de trabalho da fase $\mathrm{b}$;

$\delta_{c}$ : ciclo de trabalho da fase c;

$\Phi_{m}$ : valor máximo do fluxo de entreferro concatenado por uma fase do estator $(\mathrm{Wb})$;

$\Phi_{r}$ : módulo do fluxo magnético do rotor $(\mathrm{Wb})$;

$\Phi_{r a}$ : fluxo magnético produzido pelo rotor concatenado na fase a do estator $(\mathrm{Wb})$;

$\Phi_{r b}$ : fluxo magnético produzido pelo rotor concatenado na fase b do estator $(\mathrm{Wb})$;

$\Phi_{r c}$ : fluxo magnético produzido pelo rotor concatenado na fase c do estator $(\mathrm{Wb})$;

$\Phi_{s a}$ : fluxo magnético produzido pelo estator concatenado na fase a $(\mathrm{Wb})$;

$\Phi_{s b}$ : fluxo magnético produzido pelo estator concatenado na fase b (Wb);

$\Phi_{s c}$ : fluxo magnético produzido pelo estator concatenado na fase c $(\mathrm{Wb})$;

$\Phi_{t s a}$ : fluxo magnético total concatenado na fase a do estator $(\mathrm{Wb})$;

$\Phi_{t s b}$ : fluxo magnético total concatenado na fase b do estator $(\mathrm{Wb})$;

$\Phi_{t s c}$ : fluxo magnético total concatenado na fase c do estator $(\mathrm{Wb})$;

$\omega_{r}$ : velocidade elétrica do rotor $(\mathrm{rad} / \mathrm{s}) ;$

$\omega_{m}$ : velocidade mecânica do rotor $(\mathrm{rad} / \mathrm{s})$;

$\theta_{d q}$ : ângulo dos eixos $d q(\mathrm{rad})$;

$\theta_{m}$ : ângulo mecânico do rotor (rad); 
$\theta_{r}$ : ângulo elétrico do rotor $(\mathrm{rad})$;

$\theta_{r i}:$ valor discreto de $\theta_{r}(\mathrm{rad})$;

$\theta_{v}$ : ângulo de $v_{d q_{x}}(\mathrm{rad}) ;$

$\theta_{x}$ : ângulo do coeficiente complexo $c_{x}$ da transformação $d q$ não senoidal $(\mathrm{rad})$; 


\section{Resumo}

MONTEIRO, J. R. B. de A. Transformação dq não senoidal para máquinas síncronas com ímã permanente no rotor. São Carlos, 2002. 108p. Tese (Doutorado)

- Escola de Engenharia de São Carlos, Universidade de São Paulo.

O presente trabalho apresenta uma transformação $d q$ não senoidal e sua aplicação em máquinas síncronas com ímã permanente na superfície do rotor (MSIP) e forma de onda de FEM não senoidal. O modelo resultante da aplicação dessa transformação permite o controle direto do torque eletromagnético, permitindo reduzir as ondulações do torque produzido nesse tipo de máquina. A redução dessas ondulações em MSIPs não senoidais possibilita que esse tipo de máquina seja empregado em aplicações onde somente MSIPs senoidais, que possuem custo mais elevado, poderiam ser usadas.

Simulações e resultados práticos, obtidos pela implementação do sistema de controle vetorial não senoidal desenvolvido, são apresentados no trabalho. Pelos resultados obtidos, pode-se verificar que o método proposto é eficaz na redução das ondulações do torque eletromagnético em MSIPs não senoidais.

Palavras-chave: Acionamentos de máquinas elétricas; Máquinas elétricas; Máquinas síncronas com ímã permanente; Controle vetorial; Motor brushless $D C$; Transformação dq não senoidal. 


\section{Abstract}

MONTEIRO, J. R. B. de A. Non-sinusoidal dq transformation for permanent magnet synchronous machines. São Carlos, 2002. 108p. Tese (Doutorado) - Escola de Engenharia de São Carlos, Universidade de São Paulo.

This work presents a non-sinusoidal dq transformation and its application in permanent-magnet synchronous machines (PMSM) with a non-sinusoidal back-EMF waveform. The resulting model of the application of such transformation permits direct control of machine electromagnetic torque, which achieves the reduction of torque ripple. The reduction of torque ripple in non-sinusoidal PMSMs makes possible the use of this kind of PMSM instead of sinusoidal PMSMs, which are more expensive.

Simulation and practical results, obtained by the implementation of such non-sinusoidal vectorial control system, are shown in this work. Those results show that the method presented is efficacious in the torque ripple reduction in non-sinusoidal PM synchronous machines.

Keywords: Electrical machine drives; Electrical machines; Permanent-magnet synchronous machines; Vectorial control; Brushless DC motor; Non-sinusoidal dq transformation. 
Parte I

\section{Introdução}




\section{Capítulo 1}

\section{O Motor Síncrono com Ímã}

\section{Permanente no Rotor}

As máquinas síncronas com ímã permanente no rotor (MSIP) são largamente utilizadas em sistemas de acionamento de freqüência (ou velocidade) variável de alto desempenho.

As MSIPs apresentam uma série de vantagens em relação aos acionamentos convencionais de velocidade variável que empregam motores CC. Isso se deve ao fato das MSIPs terem um desempenho melhor do que máquinas CC. Primeiramente, as MSIPs não possuem escovas, portanto todas as desvantagens que o uso desse tipo de componente apresenta não existe nas MSIPs, como a necessidade de manutenção periódica nas escovas, centelhamento, alta emissão eletromagnética, restrição de utilização em ambientes com gases explosivos, etc.

Quanto ao tipo de rotor empregado, podemos classificar as MSIPs em dois grupos: máquinas síncronas com ímã permanente no interior do rotor e máquinas síncronas com ímã permanente na superfície do rotor.

Existem alguns tipos diferentes de rotor utilizados em máquinas síncronas com ímã permanente no interior do rotor (Monteiro, 1997) (Nasar et al., 1993). São eles:

- rotor com ímã permanente interno longitudinal;

- rotor com ímã permanente interno transversal;

- rotor Lundell com ímã permanente. 
O rotor com ímã permanente na superfície é o tipo de rotor mais empregado na prática. Esse tipo de rotor possui baixo momento de inércia devido ao fato de ser oco (Miller, 1993), o momento de inércia do rotor é cerca de um terço do momento de inércia do rotor empregado em máquinas CC. MSIPs que empregam esse tipo de rotor possuem menor peso e menor volume em relação a máquinas $\mathrm{CC}$ de mesma potência (Oliveira Jr., 1990).

Uma outra vantagem, é que esse tipo de rotor apresenta uma variação de relutância muito pequena, em função da variação do ângulo da posição rotórica (em relação ao circuito magnético do estator), sendo desprezada pela maioria dos autores (Grenier \& Louis, 1995) (Petrović et al., 2000).

Como desvantagem, a máquina que emprega esse tipo de rotor possui velocidade máxima de operação mais baixa do que as máquinas que empregam os outros tipos de rotor, devido à força de retenção dos ímãs na superfície do rotor.

As MSIPs também podem ser classificadas de acordo com a forma de onda de FEM de estator, produzida pela variação do fluxo magnético concatenado pelas espiras do estator, produzido exclusivamente pelos ímãs do rotor, ou seja, pela forma de onda da distribuição da densidade de fluxo magnético de entreferro:

- máquina síncrona com ímã permanente no rotor com forma de onda de FEM senoidal;

- máquina síncrona com ímã permanente no rotor com forma de onda de FEM não senoidal (ou comumente denominada por FEM trapezoidal).

Na literatura consultada, a máquina síncrona com ímã permanente no rotor com forma de onda de FEM senoidal é simplesmente denominada por "máquina síncrona com ímã permanente".

A máquina síncrona com ímã permanente no rotor com forma de onda de FEM não senoidal é normalmente comercializada juntamente com seu conversor de potência, sendo que, ao conjunto resultante é dado o nome de "motor brushless DC". Isso se deve ao fato do conjunto motor-conversor se comportar como uma máquina CC, mas com comutador eletrônico.

Nesse trabalho, trataremos como máquina síncrona com ímã permanente no 
rotor (MSIP), ambos os tipos citados acima, e que empregam rotor com ímã permanente na superfície. A análise do conversor convencional utilizado em sistemas "brushless DC" (conversor no modo "seis-pulsos" ou "six-step") é realizada no trabalho apenas por motivos de comparação com o método proposto.

\section{$1.1 \quad$ Objetivos}

Esse trabalho apresenta uma transformação de coordenadas que é aplicada a MSIPs com fluxo de entreferro não senoidal (transformação $d q$ não senoidal ou transformação $d q_{x}$, como apresentada no capítulo 3). Embora a transformação $d q$ não senoidal seja aplicada a MSIPs não senoidais, a aplicação a MSIPs senoidais implica somente em uma particularização da solução das coordenadas, unificando-se assim a teoria matemática que rege o comportamento de máquinas síncronas com ímã permanente na superfície do rotor (seção 3.3.1).

O principal objetivo desse trabalho é o desenvolvimento de um método para a redução das ondulações do torque eletromagnético em máquinas síncronas com ímã permanente no rotor. Ondulações no torque eletromagnético causam deterioração no desempenho geral do sistema, causam flutuações de velocidade, vibrações, são uma fonte de ruído acústico e causam imperfeições na superfície de peças usinadas. Contribuem também para o aumento das perdas do sistema.

Nesse sentido, foi desenvolvida uma transformação dq não senoidal (denominada também por transformação $d q_{x}$, nesse trabalho), que permite o tratamento dessas indesejáveis ondulações no torque eletromagnético, nesse tipo de máquina. A aplicação dessa transformação em MSIPs não senoidais é objeto de estudo desse trabalho.

\subsection{Divisão dos Capítulos}

No capítulo seguinte, uma análise das principais obras que tratam da redução das ondulações no torque eletromagnético é realizada.

No capítulo 3 , a transformação $d q$ não senoidal e as equações matemáticas do modelo vetorial da MSIP são apresentadas.

No capítulo 4, algumas simulações da MSIP são apresentadas, utilizando o mo- 
delo vetorial proposto, em malha fechada e em malha aberta de corrente. Algumas simulações da máquina com conversor no modo "seis-pulsos" são mostradas.

No capítulo 5, os resultados da implementação do controle vetorial em malha aberta de corrente, utilizando o modelo vetorial para MSIPs não senoidais, são apresentadas.

As conclusões encontram-se no capítulo 6 .

No apêndice A, é apresentado um resumo das principais técnicas de projeto adotadas para a construção de MSIPs com baixas ondulações de torque eletromagnético.

No apêndice B é realizada a dedução passo a passo das equações por fase da MSIP.

O apêndice $\mathrm{C}$ trata das transformações de coordenadas, já encontradas na literatura, e de modelos vetoriais de MSIPs, também já apresentadas na literatura consultada.

No apêndice D, são mostradas as deduções passo a passo das equações do capítulo 3 e do apêndice C.

A descrição da MSIP não senoidal utilizada no trabalho é vista no apêndice E.

O hardware utilizado para o controle e acionamento da MSIP é visto no apêndice F.

O programa executado pelo processador do hardware de controle é explicado no apêndice G. 


\section{Capítulo 2}

\section{Redução das Ondulações no Torque Eletromagnético}

Esse capítulo apresenta as principais técnicas de redução de ondulações no torque eletromagnético em MSIPs, encontradas na literatura consultada, e discute algumas particularidades do método proposto em relação aos métodos encontrados na literatura.

\subsection{Técnicas Utilizadas para Redução das Ondulações}

Pode-se dividir as técnicas de redução das ondulações de torque eletromagnético em dois grupos (Jahns, 1996):

- Técnicas de projeto de MSIPs;

- Técnicas de controle de MSIPs.

Uma breve discussão das técnicas de projetos de MSIPs é realizada no apêndice A.

As técnicas de projeto de MSIPs proporcionam uma boa redução nas ondulações do torque eletromagnético. Muitas vezes, somente essas técnicas de projeto são suficientes para que se atinja os níveis desejados de ondulações no torque eletromagnético. A grande desvantagem de se utilizar técnicas de projeto para redução das ondulações no torque eletromagnético de MSIPs é que muitas vezes elas tornam a MSIP complexa, de difícil montagem e de custo elevado. Quando essas técnicas não são suficientes, 
ou tornam-se inviáveis, recorre-se a técnicas de controle da máquina; essas técnicas dividem-se em (Jahns, 1996):

1. Controle de forma de onda de corrente programada - consiste em uma das mais populares técnicas de controle; procura-se aplicar uma forma de onda de corrente pré-determinada a fim de se cancelar as pulsações no torque eletromagnético (Berendsen et al., 1993) (Grenier \& Louis, 1995).

2. Estimadores e observadores - as técnicas por controle de forma de onda de corrente podem apresentar algumas limitações que tentam ser resolvidas por observadores e estimadores, tratando as diferentes fontes de ondulações no torque de uma maneira diferenciada.

3. Minimização do torque de comutação - essas técnicas tentam minimizar as perturbações que as comutações de fase causam no torque eletromagnético (para MSIPs não senoidais).

4. Rejeição de perturbações no laço de velocidade - consistem em técnicas de controle no laço de velocidade que agem no sentido de tentar minimizar as variações não desejadas de velocidade causadas pelas diferentes fontes de ondulações no torque eletromagnético; essas técnicas possuem boa eficácia em baixas velocidades (Holtz \& Springob, 1996).

5. Saturação do regulador de corrente em alta velocidade - essa técnica é utilizada para se conseguir altas velocidades, com baixas ondulações no torque eletromagnético, pois quando se utilizam reguladores de corrente para a alimentação de MSIPs, as ondulações no torque eletromagnético em altas velocidades tornamse críticas devido à saturação do controlador de corrente, o que pode acarretar em um alto nível de ruído acústico (Morimoto et al., 1994) (Takahashi et al., 1994) (Rahman et al., 1998).

O torque eletromagnético de uma MSIP é produzido por três fontes distintas (French, 1996). As componentes do torque eletromagnético são:

- torque de borda (ou cogging torque) - causado pela interação das bordas dos ímãs com as ranhuras do estator; 
- torque de relutância - causado pela variação da relutância do circuito magnético do motor com a posição do rotor; normalmente, essa componente é muito pequena em relação à componente anterior, por isso, é desprezada na maioria dos casos; seu tratamento não é considerado nesse trabalho;

- torque mútuo - produzido pelo acoplamento da força magnetomotriz do estator com o campo produzido pelos ímãs do rotor.

Em (French, 1996), utiliza-se uma combinação das técnicas dos ítens 1 e 2. Nesse estudo, é apresentada uma técnica para se calcular a forma de onda de corrente de referência (técnica de controle de forma de onda de corrente programada) levando-se em conta efeitos de saturação da máquina e harmônicos da FEM. No trabalho, também foi utilizado algoritmo para a estimação da posição do rotor (French \& Acarnley, 1996). Utiliza-se um estimador para se calcular o fluxo magnético concatenado.

Em (Chung et al., 1998), utilizam-se as técnicas mostradas nos ítens 2 e 3. Nesse trabalho, as ondulações no torque são tratadas em regiões de baixa velocidade, que segundo o trabalho, é a região mais crítica. No artigo, um sistema para a estimação do fluxo concatenado pelo estator é apresentado. Com o fluxo concatenado, calcula-se o torque eletromagnético desenvolvido pela máquina. O torque é controlado por um controle de estrutura variável conjugado a um PWM de espaço vetorial, que minimiza os chaveamentos nas fases da máquina, ajudando a reduzir ondulações no torque. $\mathrm{O}$ método proposto baseia-se no controle dinâmico do torque eletromagnético (ítem 2) e não do controle da corrente de estator (ítem 1), dessa forma, o controlador pode se adaptar a tipos diferentes de MSIPs.

Em (Berendsen et al., 1993), concluí-se que MSIPs trapezoidais podem ser utilizadas em aplicações de posicionamento tão bem quanto MSIPs senoidais. Para isso, é utilizada a técnica de controle de forma de onda de corrente programada. São realizadas considerações sobre as formas de onda de FEM reais e ideais.

Em (Sebastian \& Gangla, 1996), procura-se a redução nas ondulações do torque eletromagnético através do projeto da máquina. Nesse trabalho, é apresentado que o torque de borda pode ser reduzido para menos de $0,5 \%$ do torque nominal do motor, pela utilização de ranhuras inclinadas (com inclinação igual à distância entre duas ranhuras consecutivas). Portanto, o maior objetivo do trabalho é o de conseguir a redução 
do torque pulsante pela redução dos harmônicos na FEM de uma MSIP trapezoidal. Cálculos são apresentados utilizando-se técnicas de modelagem por elementos finitos, bem como cálculos empíricos para a determinação da forma de onda da FEM. Um estudo da variação de alguns parâmetros e seus efeitos na forma de onda da FEM é realizado.

Em (Holtz \& Springob, 1996), é apresentado um controle no laço de velocidade para a minimização da componente pulsante do torque eletromagnético de uma MSIP senoidal. O controlador identifica os parâmetros da máquina de forma automática e compensa os harmônicos do torque eletromagnético da máquina através de um algoritmo de detecção.

Tomando-se como base o modelo apresentado em (French, 1996), são descritos três tipos distintos de torque eletromagnético produzidos em uma MSIP: o torque de borda, o torque de relutância e o torque mútuo, conforme explicados anteriormente. Levandose em consideração esses três tipos de torque e o conversor elétrico utilizado para o acionamento de MSIPs, as ondulações no torque eletromagnético são causadas pelas seguintes fontes distintas:

- devido ao torque de borda;

- devido ao torque de relutância;

- devido aos harmônicos indesejáveis do fluxo concatenado pelo estator (harmônicos indesejáveis na forma de onda da FEM);

- devido ao desbalanceamento de fase.

- devido à estratégia de PWM utilizado;

- devido ao chaveamento das fases do estator pelo conversor elétrico não ser ideal (somente no caso de máquinas trapezoidais acionadas por inversores no modo "seis-pulsos");

Quanto ao torque de relutância, em alguns trabalhos é citado o fato de que, devido ao grande entreferro que as MSIPs possuem (com ímã na superfície do rotor), esta componente do torque pode ser desprezada. Somando-se a esse fato, as técnicas modernas utilizadas na construção do rotor reduzem a variação da relutância em função da posição do rotor (Nasar et al., 1993). 
Quanto ao chaveamento das fases do estator pelo conversor elétrico, esta componente só existe em máquinas trapezoidais onde o conversor funciona no modo clássico de "seis-pulsos" (120 - para máquinas trifásicas).

Quanto ao PWM utilizado, a freqüência limitada e a possível geração de subharmônicos faz com que se produzam ondulações indesejáveis no torque eletromagnético.

Quanto ao desbalanceamento de fase, isto ocorre devido ao processo de fabricação não ser ideal, ou seja, apresentar tolerâncias de montagem e usinagem mecânica das peças. Isso pode ocorrer tanto no estator (montagem de enrolamentos, corte de ranhuras, montagem das chapas, etc.) quanto no rotor (possíveis excentricidades, colocação dos ímãs, magnetização não uniforme, etc.)

Em (Chung et al., 1998), considera-se que as ondulações no torque sejam produzidas pelo torque de borda, harmônicos no fluxo magnético concatenado, desbalanceamento de fase e offset de corrente. Ness trabalho, o torque de relutância não é citado.

Em (Holtz \& Springob, 1996), considera-se que as fontes de produção de ondulações no torque sejam: harmônicos indesejáveis na FEM, torque de borda e desbalanceamento de fase, são denominados por "efeitos secundários" nesse trabalho.

Em (Berendsen et al., 1993), desenvolve-se um método de compensação das ondulações no torque por uma referência de corrente. Nesse trabalho, considera-se apenas os efeitos de chaveamento de fase e de harmônicos indesejáveis na forma de onda da FEM. O torque de borda e o torque de relutância não são considerados.

Em (Grenier \& Louis, 1995), somente os harmônicos indesejáveis na forma de onda da FEM são tratados. No trabalho, é apresentado um método para a determinação de uma corrente de referência.

Em (Sebastian \& Gangla, 1996), considera-se o torque de borda, os harmônicos indesejáveis na forma de onda da FEM e o chaveamento de fase realizado pelo conversor. Outros efeitos, como o torque de relutância, são desconsiderados.

Em (Grenier et al., 1997), é realizada uma análise de uma MSIP com forma de onda de FEM senoidal, com variação de relutância significativa. Mas, a variação de relutância é significativa devido ao fato da máquina possuir rotor com ímã permanente interno. No trabalho, a componente de corrente direta (da decomposição $d q$ ) é utilizada para a compensação da variação de relutância, ou seja, o torque de relutância da máquina é 
explorado a fim de se atingir o ponto ótimo de operação, máximo torque eletromagnético e baixas perdas.

Em (Park et al., 2000), é apresentado um método para o cálculo das correntes ótimas de fase em uma MSIP. Essa referência de corrente é calculada com o objetivo de se reduzir as ondulações no torque eletromagnético e de reduzir as perdas na máquina. O método proposto considera assimetrias da máquina e condições de desbalanceamento de fase. O trabalho trata do torque de relutância e do torque de borda como sendo desprezíveis.

Em (Le-Huy et al., 1986), é apresentado um método para a redução das ondulações no torque eletromagnético através da eliminação dos harmônicos do torque eletromagnético pela injeção de harmônicos nas correntes de estator.

Em (Petrović et al., 2000), é proposta uma alternativa para a estimação das formas de onda da densidade de fluxo magnético no entreferro pela parametrização das expressões de fluxo. O trabalho emprega a combinação das técnicas 1 e 2 . No trabalho, é considerado que os harmônicos que produzem ondulações no torque são múltiplos de 6, inclusive os harmônicos do fluxo magnético. Considera que o torque de relutância e o torque de borda são desprezíveis, para máquinas com pólos não salientes, sendo que a minimização do torque de borda é feita no projeto da máquina. A máquina considerada é uma máquina com pólos salientes, portanto, possui valor de torque de relutância significativo. No entanto, a forma de onda da relutância em função da posição rotórica é considerada senoidal, sendo que não constitui uma fonte de ondulações no torque e, mesmo nessa máquina, a magnitude do torque de relutância é pequena frente à magnitude do torque mútuo. Embora a máquina utilizada apresente um torque de borda desprezível, o trabalho considera que em outros tipos de MSIPs esse pode ter um valor significativo.

Em (Aghili et al., 2000), é apresentada uma estratégia para o controle do torque livre de oscilações em malha aberta de velocidade, utilizando uma modelagem no domínio da freqüência por séries de Fourier. O trabalho também procura a diminuição das perdas no cobre para a geração de torque em altas velocidades. Um fato interessante a ser destacado é que, no trabalho, cita-se: "A principal tendência no controle de motores síncronos é baseada na transformação das correntes via transformação $d q$. Uma vez que a transformação lineariza somente um motor ideal com força magnetomotriz distribuída 
de forma perfeitamente senoidal, uma outra topologia de compensação de torque deve ser usada em cascata para o cancelamento das ondulações de torque." Isso torna o presente trabalho muito interessante, uma vez que apresenta uma modelagem vetorial com uma transformação $d q$ não senoidal para MSIPs não senoidais, não sendo necessária nenhuma outra topologia adicional para a compensação das ondulações do torque.

Na maioria dos trabalhos, o torque de relutância não é considerado como significativo para a produção de ondulações no torque eletromagnético da máquina (em máquinas com ímã permanente na superfície do rotor). Isto ocorre porque, atualmente, as MSIPs com ímã na superfície do rotor são construídas para que apresentem uma variação mínima na relutância do circuito magnético do estator com a posição do rotor, sendo que isso é facilitado pela colocação das pastilhas magnéticas na superfície do rotor, o que resulta em um entreferro relativamente grande (Nasar et al., 1993).

\subsection{Particularidades do Método Proposto}

O método proposto no presente trabalho pode ser classificado conforme o ítem 1 (Controle de forma de onda de corrente programada). Trabalhos que empregam essa técnica são (Berendsen et al., 1993), (French, 1996), (Grenier \& Louis, 1995), (Park et al., 2000) e (Petrović et al., 2000). O método proposto nesse trabalho apresenta vantagens sobre os métodos propostos em (Berendsen et al., 1993) e (French, 1996), por permitir a minimização das perdas no cobre $^{1}$ (Grenier \& Louis, 1995) e pela possibilidade de enfraquecimento ou enriquecimento de campo, por tratar-se de um modelo vetorial. Isso porque o método de controle vetorial permite que se controle separadamente as componentes da corrente de estator: a componente que produz torque eletromagnético (corrente de quadratura) e a componente que é responsável pelo enfraquecimento ou enriquecimento do campo (corrente direta).

O método proposto consiste em uma variação do método apresentado em (Grenier \& Louis, 1995) ${ }^{2}$, consistindo na determinação de um sistema de coordenadas onde a componente da corrente de estator que produz torque é isolada e o torque eletromagnético é diretamente proporcional a essa componente; dessa forma, o método possui a van-

\footnotetext{
${ }^{1} \mathrm{~A}$ minimização das perdas no cobre é discutida na seção 3.3.2.

${ }^{2} \mathrm{O}$ método apresentado em (Grenier \& Louis, 1995) é explicado no apêndice C, equação C.5 e seção C.2.3.
} 
tagem de tornar mais simples a equação do torque eletromagnético, do que o método apresentado em (Grenier \& Louis, 1995). Uma outra vantagem é a possibilidade do tratamento da máquina em regime permanente de torque eletromagnético, em malha aberta de corrente, como é mostrado no capítulo 4. 


\section{Parte II}

\section{Desenvolvimento}




\section{Capítulo 3}

\section{Modelagem Matemática}

A transformação $d q$ não senoidal e o modelo vetorial proposto para o controle e acionamento de máquinas síncronas com ímã permanente na superfície do rotor são apresentados nesse capítulo. Primeiramente, apresenta-se o modelo por fase da máquina. Esse modelo é bem conhecido e a dedução das equações desse modelo pode ser acompanhada no apêndice B. O objetivo da apresentação desse modelo no presente capítulo é o de familiarizar o leitor com a notação matemática utilizada no trabalho.

\subsection{Modelagem Convencional por Fase}

Na modelagem convencional por fase, o modelo do motor é obtido considerandose a estrutura física da máquina. A equação elétrica da máquina (equação 3.1) e do torque eletromagnético (equação 3.2) consideram a corrente e a tensão em cada fase da máquina. As equações mostradas referem-se a máquinas trifásicas e, nesse caso, ligadas em estrela (ou "Y"). As equações para máquinas com maior número de fases podem ser obtidas de modo semelhante.

Para o desenvolvimento das equações, algumas hipóteses são consideradas:

- variação da relutância no estator devido à posição do rotor é desprezível;

- as propriedades magnéticas e elétricas dos materiais utilizados são lineares, dentro da região de operação normal da máquina;

- simetria entre as resistências, auto-indutâncias e indutâncias mútuas das fases do 


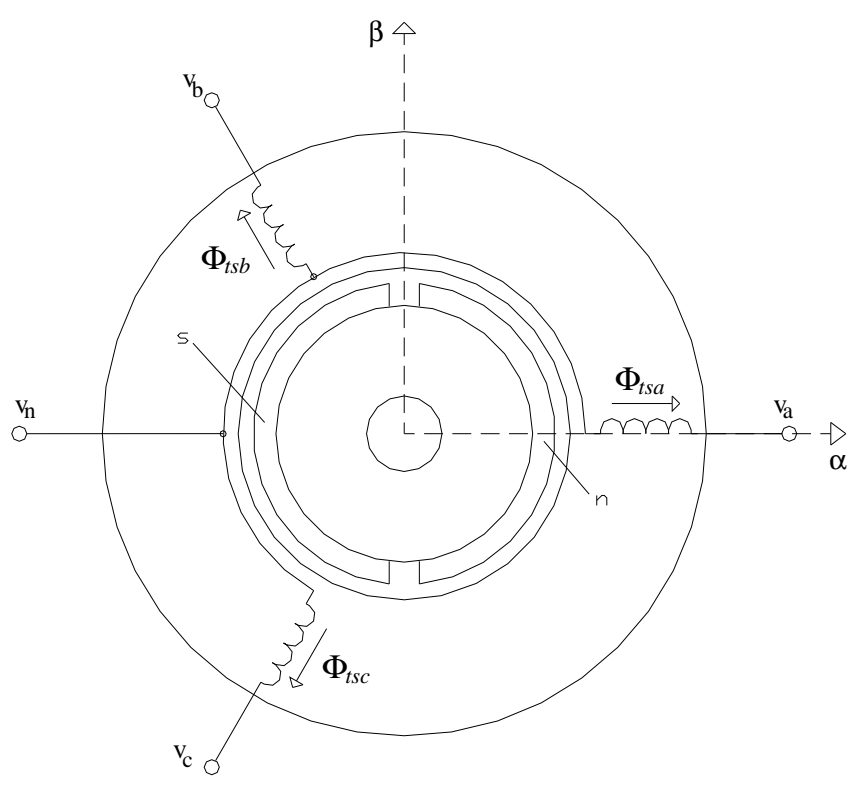

Figura 3.1: Esquema físico simplificado do servo-motor (motor com 2 pólos).

estator.

A título de exemplificação, na figura 3.1 é apresentado um esquema simplificado de uma máquina que atende as hipóteses acima. Trata-se de uma máquina trifásica com 1 par de pólos. Na figura, o rotor é mostrado em sua posição de origem.

$$
\begin{gathered}
{\left[\begin{array}{c}
v_{a} \\
v_{b} \\
v_{c}
\end{array}\right]=R_{s}\left[\begin{array}{c}
i_{a} \\
i_{b} \\
i_{c}
\end{array}\right]+L \frac{d}{d t}\left[\begin{array}{c}
i_{a} \\
i_{b} \\
i_{c}
\end{array}\right]+\left[\begin{array}{c}
e_{a} \\
e_{b} \\
e_{c}
\end{array}\right]+\left[\begin{array}{l}
v_{n} \\
v_{n} \\
v_{n}
\end{array}\right]} \\
T_{e l}=z_{p} \Phi_{m}\left[\begin{array}{c}
i_{a} \\
i_{b} \\
i_{c}
\end{array}\right]^{t}\left[\begin{array}{c}
F_{r a} \\
F_{r b} \\
F_{r c}
\end{array}\right]
\end{gathered}
$$

Onde:

$v_{a}, v_{b}$ e $v_{c}$ : tensões aplicadas às fases $\mathrm{a}, \mathrm{b} \mathrm{e} \mathrm{c}$ do estator, respectivamente (figura 3.1);

$i_{a}, i_{b}$ e $i_{c}$ : correntes nas fases a, b e c do estator, respectivamente; $e_{a}, e_{b}$ e $e_{c}$ : tensões induzidas nas fases $\mathrm{a}, \mathrm{b}$ e c do estator devido ao movi- 
mento do rotor e ao campo gerado pelos ímãs do rotor, respectivamente;

$v_{n}$ : tensão do terminal neutro do estator (esse terminal não é conectado na prática, figura 3.1);

$R_{s}$ : resistência de uma fase do estator;

$T_{e l}$ : torque eletromagnético desenvolvido pela máquina;

$z_{p}$ : número de pares de pólos da máquina;

$\Phi_{m}$ : valor máximo atingido pelo campo magnético, produzido exclusivamente pelos ímãs do rotor, concatenado pelas espiras das fases do estator;

$F_{r a}, F_{r b}$ e $F_{r c}$ : formas de onda de FEM, produzidas exclusivamente pelos ímãs do rotor, normalizadas, escritas em função da posição do rotor $\left(\theta_{r}\right)$;

$L$ : matriz de indutâncias do estator, definida na equação 3.3.

$$
L=\left[\begin{array}{ccc}
L_{s} & M_{s} & M_{s} \\
M_{s} & L_{s} & M_{s} \\
M_{s} & M_{s} & L_{s}
\end{array}\right]
$$

Onde:

$L_{s}$ : auto-indutância de uma fase do estator;

$M_{s}$ : indutância mútua entre duas fases do estator.

\subsection{Transformação $d q$ Não Senoidal}

A equação completa da transformação $d q$ não senoidal pode ser vista na equação 3.4 .

$$
x_{\alpha \beta}=c_{x} e^{j \theta_{r}} x_{d q_{x}}
$$

Essa transformação consiste em uma transformação $d q$, com o ângulo dos eixos $d q$ igual ao ângulo do rotor $\left(\theta_{r}\right)$, seguida da aplicação de um coeficiente complexo (equação 3.5 seguida da equação 3.6$)$. 


$$
\begin{gathered}
x_{\alpha \beta}=e^{j \theta_{r}} x_{d q} \\
x_{d q}=c_{x} x_{d q_{x}}
\end{gathered}
$$

Sendo:

$$
\begin{aligned}
& c_{x} \in \mathbf{C}^{*} \\
& c_{x}=a_{x} e^{j \theta_{x}} \\
& a_{x} \in \mathbf{R}^{*} \\
& \theta_{x} \in \mathbf{R}
\end{aligned}
$$

Onde:

$$
\begin{aligned}
& a_{x} \text { e } \theta_{x}: \text { módulo e ângulo de } c_{x} ; \\
& x_{\alpha \beta}: \text { grandeza representada nos eixos } \alpha \beta ; \\
& x_{d q}: \text { grandeza representada nos eixos } d q ; \\
& x_{d q_{x}}: \text { grandeza representada nos eixos } d q_{x} ; \\
& \theta_{r} \text { : ângulo do rotor, posição elétrica. }
\end{aligned}
$$

No caso de MSIPs não senodais, é importante notar que as grandezas $c_{x}, a_{x}$ e $\theta_{x}$ não são constantes, mas sim, função da posição do rotor da máquina $\left(\theta_{r}\right)$.

Graficamente, os três eixos de coordenadas estão representados na figura 3.2, onde pode-se ver que a amplitude dos eixos $d q_{x}$ é dada pela grandeza $a_{x}$, em relação aos outros dois eixos, $\alpha \beta$ e $d q$, unitários.

Um diagrama em blocos representando a transformação $d q$ é visto na figura 3.3. No diagrama, as grandezas representadas nos eixos $d q$ são convertidas para as coordenadas $\alpha \beta$. A transformação $d q$ não senoidal é mostrada na figura 3.4, onde as grandezas representadas nos eixos $d q_{x}$ são transformadas nas grandezas representadas nos eixos 


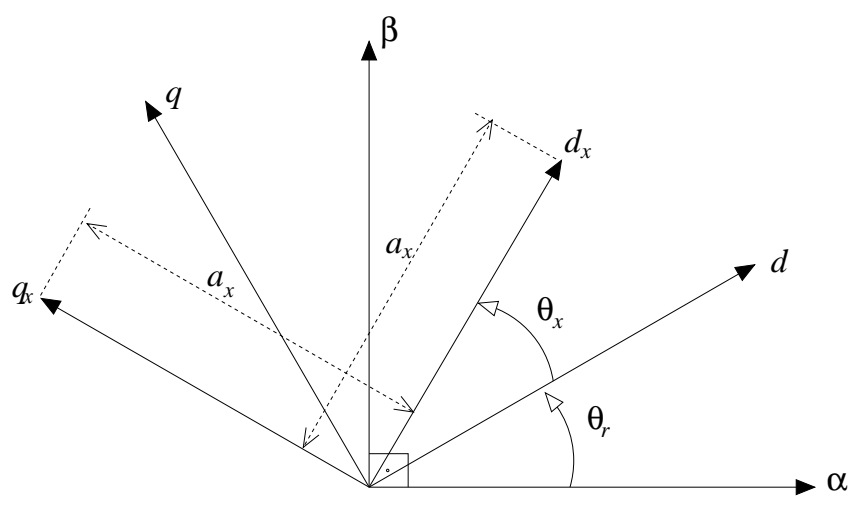

Figura 3.2: Representação gráfica dos três eixos de coordenadas utilizados nas transformações aplicadas à MSIP.

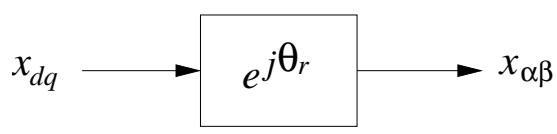

Figura 3.3: Diagrama em blocos da transformação $d q$.

$\alpha \beta$. A transformação das grandezas representadas nos eixos $d q_{x}$ para as representadas nos eixos $d q$ é mostrada na figura 3.5.

\subsection{Modelo Vetorial da MSIP}

Para a obtenção do modelo vetorial da MSIP, é necessária a utilização de duas transformações de coordenadas nas equações de fase da máquina (equações 3.1 e 3.2). A primeira transformação a ser aplicada ao modelo por fase da máquina é a transformação $\alpha \beta 0$, seguida da transformação $d q$ não senoidal.

A transformação $\alpha \beta 0$ é vista no apêndice C, equações C.1 e C.2. Com a aplicação dessa transformação, obtêm-se as equações elétricas e de torque eletromagnético da máquina, no sistema de coordenadas $\alpha \beta 0$, equações C.6, C.7 e C.8.

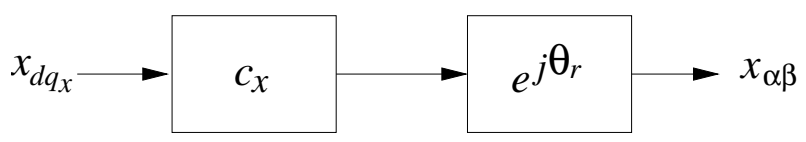

Figura 3.4: Diagrama em blocos da transformação $d q$ não senoidal completa. 


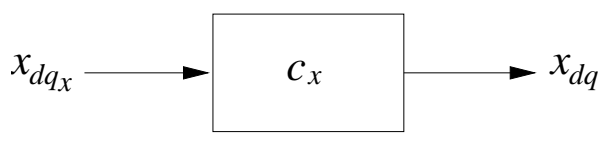

ou

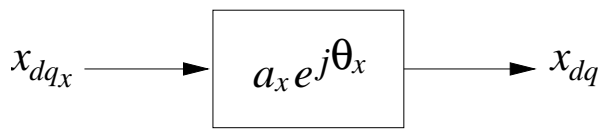

Figura 3.5: Diagrama em blocos da transformação para as grandezas nos eixos $d q_{x}$ para os eixos $d q$.

A equação C.7 não será utilizada pois a máquina não possui o terminal central conectado, portanto a corrente $i_{0}$ é nula. Dessa forma, a equação do torque eletromagnético é escrita sem o termo $i_{0} F_{r 0}$ (equação C.9).

Para a obtenção das equações do modelo vetorial proposto pelo presente trabalho, aplica-se a transformação $d q$ não senoidal nas equações C.6 e C.9. A aplicação da transformação $d q$ não senoidal (equação 3.4) nas equações $\alpha \beta$ da máquina, resulta nas equações 3.8 e 3.9 .

$$
\begin{gathered}
v_{d q_{x}}=R_{s} i_{d q_{x}}+\left(L_{s}-M_{s}\right)\left(\frac{d}{d t} i_{d q_{x}}+\omega_{r} i_{d q_{x}}\left(j+\frac{1}{c_{x}} \frac{d c_{x}}{d \theta_{r}}\right)\right)+\omega_{r} \Phi_{m} F_{r d q_{x}} \\
T_{e l}=z_{p} a_{x}^{2} \Phi_{m}\left(i_{d_{x}} F_{r d_{x}}+i_{q_{x}} F_{r q_{x}}\right)
\end{gathered}
$$

Onde:

$v_{d q_{x}}$ : tensão aplicada ao estator representada nos eixos $d q_{x}$ (forma complexa:

$$
\left.v_{d_{x}}+j v_{q_{x}}\right)
$$

$i_{d q_{x}}$ : corrente de estator representada nos eixos $d q_{x}$ (forma complexa: $i_{d_{x}}+$ $\left.j i_{q_{x}}\right)$;

$F_{r d q_{x}}$ : forma de onda de FEM normalizada, representada nos eixos $d q_{x}$ (forma complexa: $F_{r d_{x}}+j F_{r q_{x}}$ ); 
Além disso, não é necessário que as formas de onda de FEM sejam simétricas entre si, pois as transformações de coordenadas suportam esse fato. Também não é necessário que as formas de onda de FEM sejam simétricas de um par de pólo a outro, pois basta utilizar-se da posição mecânica $\left(\theta_{m}\right)$, ao invés da posição elétrica do rotor $\left(\theta_{r}\right)$, para as grandezas que são função da posição do rotor.

Expandindo-se o termo $c_{x}$ para $a_{x} e^{j \theta_{x}}$, obtém-se a equação 3.10 .

$$
\begin{gathered}
v_{d q_{x}}=R_{s} i_{d q_{x}}+\left(L_{s}-M_{s}\right)\left(\frac{d}{d t} i_{d q_{x}}+\omega_{r} i_{d q_{x}}\left(\frac{1}{a_{x}} \frac{d a_{x}}{d \theta_{r}}+j\left(1+\frac{d \theta_{x}}{\theta_{r}}\right)\right)\right)+ \\
\omega_{r} \Phi_{m} F_{r d q_{x}}
\end{gathered}
$$

Com a finalidade de se simplificar a equação obtida para o torque eletromagnético da máquina, a equação 3.9, impõe-se a seguinte condição:

$$
F_{r d_{x}}=0
$$

Dessa forma, a equação do torque eletromagnético da máquina (equação 3.9) passa a depender apenas das grandezas do eixo $q_{x}$. Isso significa que a posição dos eixos $d q_{x}$ é agora determinada de tal modo que satisfaça a condição de manter $F_{r d_{x}}$ igual a zero. Isso implica que $\theta_{x}$ deve ser uma função do ângulo do rotor $\left(\theta_{r}\right)$, cuja solução está apresentada na equação 3.12. Nota-se que $\theta_{x}$ é uma função da posição do ângulo do rotor $\left(\theta_{r}\right)$.

$$
\theta_{x}=\arctan \frac{-F_{r \alpha}}{F_{r \beta}}-\theta_{r}
$$

A segunda condição a ser imposta com relação à equação do torque eletromagnético (equação 3.9) é dada pela equação 3.13, a seguir. Essa condição é imposta a fim de se obter uma equação de torque eletromagnético equivalente à obtida para a MSIP senoidal (equação C.17);

$$
a_{x}^{2} F_{r q_{x}}=\sqrt{\frac{3}{2}}
$$

Para se satisfazer a condição dada pela equação 3.13 , tem-se para $a_{x}$, a solução 
dada pela equação 3.14. Nota-se que $a_{x}$ é uma função da posição do ângulo do rotor $\left(\theta_{r}\right)$.

$$
a_{x}=\sqrt{\frac{3}{2}} \frac{1}{\sqrt{F_{r \alpha}^{2}+F_{r \beta}^{2}}}
$$

Uma vez que existem vários tipos de forma de onda de FEM para MSIPs não senodais, tal como mostrado em (Monteiro, 1997) e utilizado em (Grenier \& Louis, 1995), (Miller, 1993) e (Berendsen et al., 1993), as grandezas $a_{x}$ e $\theta_{x}$ são particulares para cada tipo de MSIP não senoidal, pois dependem da forma de onda de FEM da máquina.

Tendo-se as equações 3.12 e 3.14, pode-se reescrever as equações elétricas e de torque eletromagnético da máquina (equações 3.10 e 3.9) nas equações 3.15, 3.16 e 3.17, no domínio real.

$$
\begin{gathered}
v_{d_{x}}=R_{s} i_{d_{x}}+\left(L_{s}-M_{s}\right)\left(\frac{d}{d t} i_{d_{x}}+\omega_{r}\left(\frac{1}{a_{x}} \frac{d a_{x}}{d \theta_{r}} i_{d_{x}}-\left(1+\frac{d \theta_{x}}{d \theta_{r}}\right) i_{q_{x}}\right)\right) \\
v_{q_{x}}=R_{s} i_{q_{x}}+\left(L_{s}-M_{s}\right)\left(\frac{d}{d t} i_{q_{x}}+\omega_{r}\left(\frac{1}{a_{x}} \frac{d a_{x}}{d \theta_{r}} i_{q_{x}}+\left(1+\frac{d \theta_{x}}{d \theta_{r}}\right) i_{d_{x}}\right)\right)+ \\
\sqrt{\frac{3}{2}} \Phi_{m} \frac{1}{a_{x}^{2}} \omega_{r} \\
T_{e l}=z_{p} \sqrt{\frac{3}{2}} \Phi_{m} i_{q_{x}}
\end{gathered}
$$

Pode-se notar que a equação do torque eletromagnético (equação 3.17) é equivalente à equação do torque eletromagnético para MSIPs senoidais, mostrada no apêndice C (equação C.17). A diferença entre elas é que, no caso senoidal, utiliza-se a projeção da corrente de estator no eixo $q$, e no presente caso, utiliza-se a projeção da corrente no eixo $q_{x}$. 


\subsubsection{Particularização para o caso senoidal}

Para o caso de uma MSIP senoidal, tem-se que $\theta_{x}=0$ e $a_{x}=1$. Com $\theta_{x}$ igual a zero, o ângulo dos eixos $d q_{x}$ vai ser o mesmo do que o dos eixos $d q$ que, por sua vez, é igual ao ângulo do rotor $\left(\theta_{r}\right)$. Com $a_{x}=1$, a amplitude dos eixos $d q_{x}$ passa a ser unitária, tal como os eixos $d q$.

As equações elétricas anteriores, para o caso de MSIPs com forma de onda de FEM qualquer, são então escritas nas equações 3.18 e 3.19 para o caso senoidal. A equação do torque eletromagnético é a mesma mostrada na equação 3.17. Essas equações são semelhantes às equações apresentados no apêndice C, equações C.15, C.16 e C.17. A diferença entre elas é que as correntes de estator foram escritas nos eixos $d q_{x}$ (com o sub-índice $x$ : $i_{d_{x}}$ e $i_{q_{x}}$ ), embora tenham o mesmo valor das correntes para o caso senoidal $\left(i_{d}\right.$ e $\left.i_{q}\right)$.

$$
\begin{gathered}
v_{d_{x}}=R_{s} i_{d_{x}}+\left(L_{s}-M_{s}\right) \frac{d}{d t} i_{d_{x}}-\left(L_{s}-M_{s}\right) \omega_{r} i_{q_{x}} \\
v_{q_{x}}=R_{s} i_{q_{x}}+\left(L_{s}-M_{s}\right) \frac{d}{d t} i_{q_{x}}+\left(L_{s}-M_{s}\right) \omega_{r} i_{d_{x}}+\sqrt{\frac{3}{2}} \Phi_{m} \omega_{r}
\end{gathered}
$$

\subsubsection{Perdas no cobre}

A equação 3.17 apresenta o torque eletromagnético como sendo diretamente proporcional a uma das duas componentes da corrente de estator, portanto pode-se minimizar as perdas no cobre anulando-se a outra componente da corrente que não produz torque (anulando-se $i_{d_{x}}$ ). Dessa forma, as equações elétricas da MSIP são escritas nas equações 3.20 e 3.21 , para $i_{d_{x}}=0$.

$$
\begin{gathered}
v_{d_{x}}=-\left(L_{s}-M_{s}\right) \omega_{r}\left(1+\frac{d \theta_{x}}{d \theta_{r}}\right) i_{q_{x}} \\
v_{q_{x}}=R_{s} i_{q_{x}}+\left(L_{s}-M_{s}\right)\left(\frac{d}{d t} i_{q_{x}}+\omega_{r} \frac{1}{a_{x}} \frac{d a_{x}}{d \theta_{r}} i_{q_{x}}\right)+\sqrt{\frac{3}{2}} \Phi_{m} \frac{1}{a_{x}^{2}} \omega_{r}
\end{gathered}
$$




\subsection{Análise da MSIP em Regime Permanente}

Comparando os termos das equações 3.15 e 3.16 com os termos das equações elétricas obtidas com a transformação $d q$ (equações C.15 e C.16), pode-se concluir que:

- os termos $\left(L_{s}-M_{s}\right) \frac{d}{d t} i_{d_{x}}$, na equação 3.15 , e $\left(L_{s}-M_{s}\right) \frac{d}{d t} i_{q_{x}}$, na equação 3.16 , são devidos à variação da corrente de estator, nos eixos de coordenadas utilizados, ou seja, nos eixos $d q_{x}$

- os termos - $\left(L_{s}-M_{s}\right) \omega_{r} i_{q_{x}}$, na equação 3.15 , e $\left(L_{s}-M_{s}\right) \omega_{r} i_{d_{x}}$, na equação 3.16 , são relativos ao acoplamento entre os eixos $d_{x}$ e $q_{x}$ e ao movimento desses eixos em torno dos eixos $\alpha \beta$, como no caso senoidal (equações C.15 e C.16). Através da corrente $i_{d_{x}}$ pode-se controlar o campo da máquina: com $i_{d_{x}}$ menor que zero, realiza-se enfraquecimento do campo, podendo aumentar a velocidade final de operação da máquina, ou com $i_{d_{x}}$ maior que zero, realiza-se enriquecimento do campo;

- os termos - $\left(L_{s}-M_{s}\right) \omega_{r} \frac{d \theta_{x}}{d \theta_{r}} i_{q_{x}}$, na equação 3.15 , e $\left(L_{s}-M_{s}\right) \omega_{r} \frac{d \theta_{x}}{d \theta_{r}} i_{q_{x}}$, na equação 3.16, também são relativos aos acoplamentos entre os eixos $d_{x}$ e $q_{x}$ e devido à oscilação que esses eixos realizam em torno do ângulo do rotor $\left(\theta_{r}\right)$;

- os termos $\left(L_{s}-M_{s}\right) \omega_{r} \frac{1}{a_{x}} \frac{d a_{x}}{d \theta_{r}} i_{d_{x}}$, na equação 3.15 , e $\left(L_{s}-M_{s}\right) \omega_{r} \frac{1}{a_{x}} \frac{d a_{x}}{d \theta_{r}} i_{q_{x}}$, na equação 3.16, consistem na componente relativa à variação da amplitude dos eixos $d q_{x}$, isto é, a tensão produzida internamente devido à rotação da máquina, mesmo com as correntes $i_{d_{x}}$ e $i_{q_{x}}$ mantidas constantes;

- finalmente, o termo $\sqrt{\frac{3}{2}} \Phi_{m} \frac{1}{a_{x}^{2}} \omega_{r}$ é relativo à forma de onda de fluxo de entreferro, ou à forma de onda de FEM.

De acordo com a análise anterior, e levando-se em conta a expressão do torque eletromagnético (equação 3.17), é possível se obter um modelo para a MSIP não senoidal em regime permanente de torque.

Mantendo-se $i_{q_{x}}$ constante, pois o torque é diretamente proporcional a $i_{q_{x}}$, e $i_{d_{x}}$ igual a uma fração de $i_{q_{x}}$, ou seja ${ }^{1}$ :

\footnotetext{
${ }^{1}$ Através do valor de $k_{i x}$ pode-se controlar o enriquecimento ou enfraquecimento do campo.
} 


$$
i_{d_{x}}=k_{i x} i_{q_{x}}
$$

Pode-se escrever que $i_{q_{x}}=i_{q_{x} r e f}$ e:

$$
i_{q_{x} r e f}=T_{\text {elref }} \sqrt{\frac{2}{3}} \frac{1}{z_{p} \Phi_{m}}
$$

Nesse sentido, as equações 3.15 e 3.16 são agora descritas pelas equações 3.24 e 3.25, que descrevem o comportamento da máquina em regime permanente, ou seja, sem transitório de torque eletromagnético.

$$
\begin{gathered}
v_{d_{x}}=\left(R_{s} k_{i x}+\left(L_{s}-M_{s}\right) \omega_{r}\left(\frac{1}{a_{x}} \frac{d a_{x}}{d \theta_{r}} k_{i x}-\left(1+\frac{d \theta_{x}}{d \theta_{r}}\right)\right)\right) i_{q_{x} r e f} \\
v_{q_{x}}=\left(R_{s}+\left(L_{s}-M_{s}\right) \omega_{r}\left(\frac{1}{a_{x}} \frac{d a_{x}}{d \theta_{r}}+\left(1+\frac{d \theta_{x}}{d \theta_{r}}\right) k_{i x}\right)\right) i_{q_{x} r e f}+ \\
\sqrt{\frac{3}{2}} \Phi_{m} \frac{1}{a_{x}^{2}} \omega_{r}
\end{gathered}
$$

Com as equações acima, nota-se que é possível acionar uma MSIP não senoidal obtendo-se torque eletromagnético livre de ondulações em regime permanente, somente com a leitura da posição do rotor $\left(\theta_{r}\right)$. Isso é possível uma vez que se conheçam os parâmetros da máquina, tais como $R_{s}, L_{s}, M_{s}, \Phi_{m}, z_{p}$ e a forma de onda da FEM. 


\section{Capítulo 4}

\section{Simulação da MSIP Utilizando o}

Modelo Vetorial

Nesse capítulo, o modelo vetorial para MSIPs não senoidais, apresentado no capítulo 3, foi utilizado para a realização das simulações de um acionamento de uma MSIP não senoidal. Primeiramente, os parâmetros necessários à simulação e ao controle da máquina são levantados. Depois, realiza-se a simulação da máquina em malha fechada e em malha aberta de corrente. Para fins comparativos, algumas simulações da máquina com conversor no modo "seis-pulsos" são mostradas.

A máquina utilizada para a aplicação do modelo proposto é apresentada em detalhes no apêndice E.

\subsection{Parâmetros da Máquina}

Com a finalidade de se aplicar o modelo proposto na máquina, é necessário que se tenham os parâmetros da máquina. Os parâmetros da máquina, utilizados nas simulações, estão mostrados na tabela E.1.

Além dos parâmetros mostrados na tabela E.1, outros parâmetros foram conseguidos através de medições realizadas na máquina, como $\Phi_{m}, F_{r a}, F_{r b}$ e $F_{r c}$. Com o objetivo de se determinar tais parâmetros, a máquina foi girada externamente e as tensões de linha da máquina foram medidas. A figura 4.1 mostra as tensões de linha da máquina. Essas tensões de linha são geradas pelo campo magnético produzido 


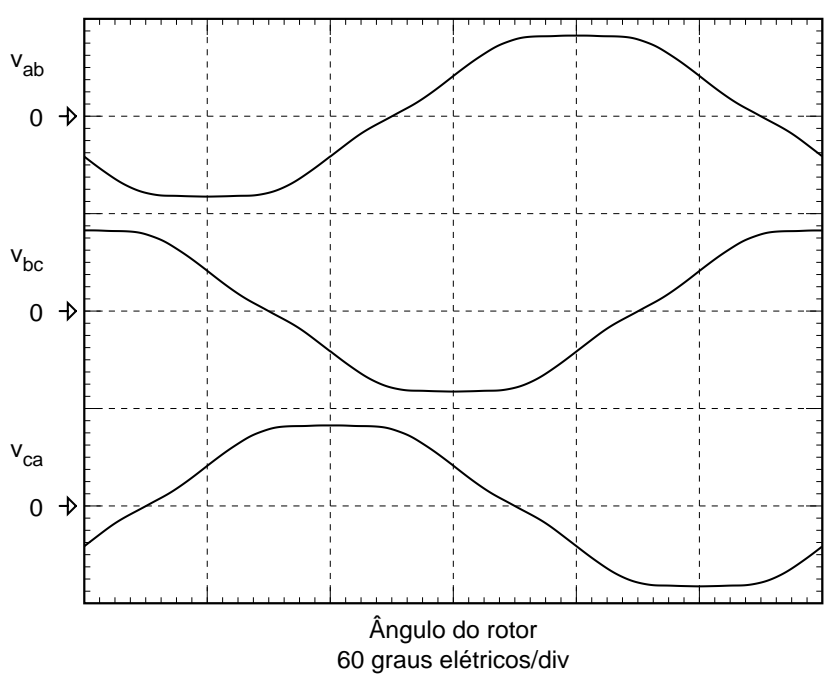

Figura 4.1: Tensão de linha da máquina, geradas pela variação do fluxo magnético concatenado pelas espiras do estator, produzido exclusivamente pelos ímãs do rotor.

exclusivamente pelos ímãs do rotor.

Com as tensões de linha da máquina, chega-se às formas de onda de tensão induzida, ou seja, a $F_{r a}, F_{r b}$ e $F_{r c}$, mostradas na figura 4.2. Com isso, obtém-se o valor de $\Phi_{m}$, que para a máquina utilizada é de $0,12 \mathrm{~V} \cdot \mathrm{s} / \mathrm{rad}$.

Com base nas formas de onda de tensão induzida na máquina $\left(F_{r a}, F_{r b}\right.$ e $\left.F_{r c}\right)$, determina-se os demais parâmetros a fim de se completar seu modelo vetorial, que são: $a_{x}, \theta_{x}, \frac{d a_{x}}{d \theta_{r}}$ e $\frac{d \theta_{x}}{d \theta_{r}}$. Esses parâmetros podem ser vistos nas figuras 4.3, 4.4, 4.5 e 4.6, respectivamente.

A equação mecânica, utilizada nas simulações, é a equação 4.1, cujos parâmetros são mostrados na tabela 4.1.

A tensão de barramento utilizada nas simulações é de $150 \mathrm{~V}\left(V_{B U S}\right)$.

$$
T_{e l}=\left(J_{m}+J_{c}\right) \frac{d \omega_{m}}{d t}+\left(B_{m}+B_{c}\right) \omega_{m}+T_{c}
$$

Onde:

$\omega_{m}$ : velocidade mecânica do rotor;

$J_{m}:$ momento de inércia do rotor da máquina; 


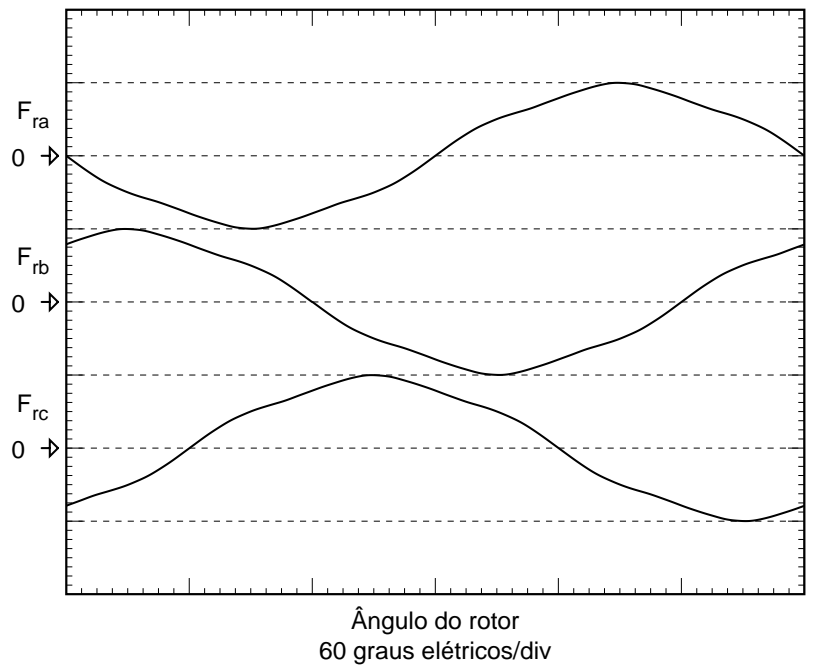

Figura 4.2: Forma de onda de tensão induzida da máquina, $F_{r a}, F_{r b}$ e $F_{r c}$, com amplitude unitária.

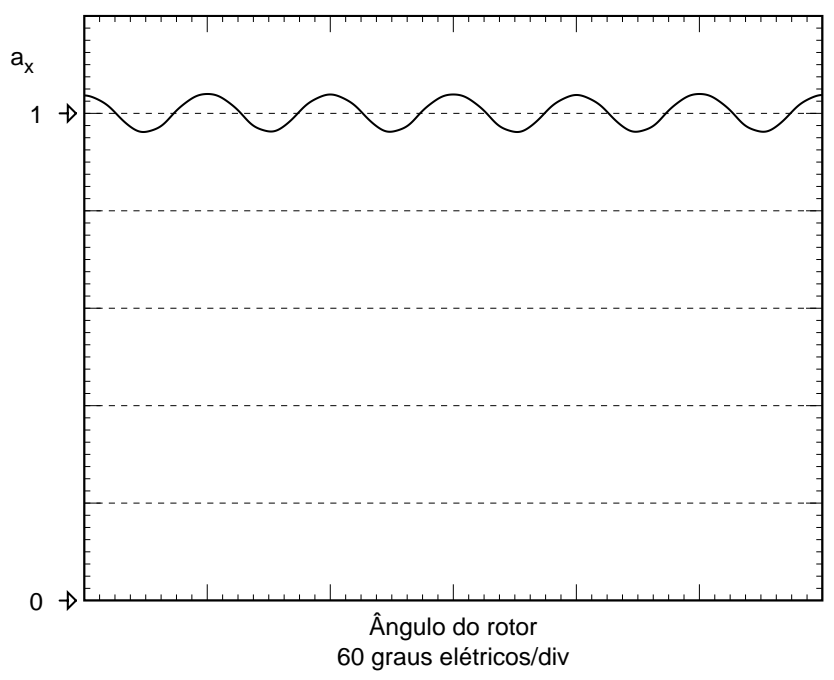

Figura 4.3: Parâmetro $a_{x}$ para a máquina utilizada, em função da posição rotórica $\left(\theta_{r}\right)$. 


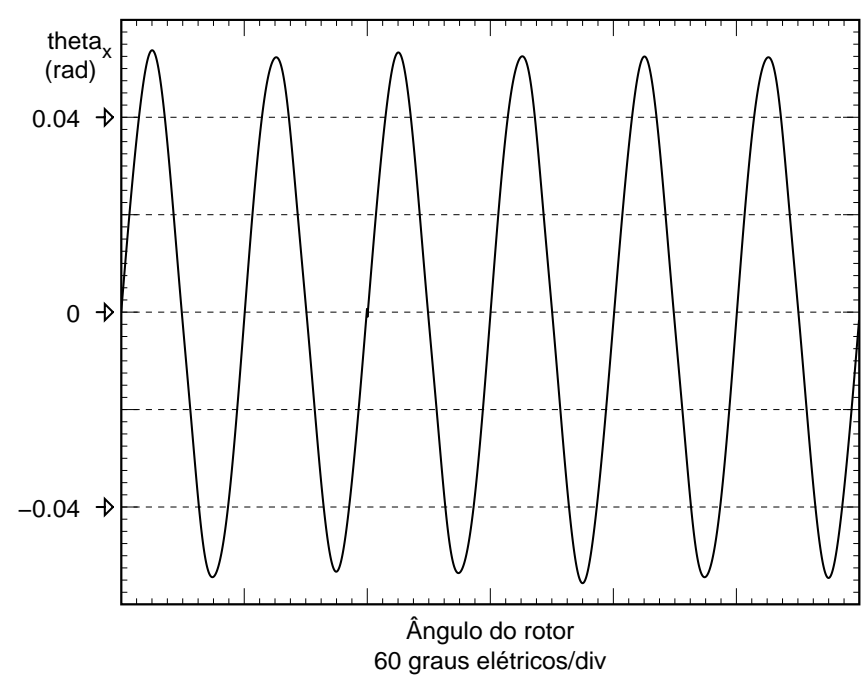

Figura 4.4: Parâmetro $\theta_{x}$ para a máquina utilizada, em função da posição rotórica $\left(\theta_{r}\right)$.

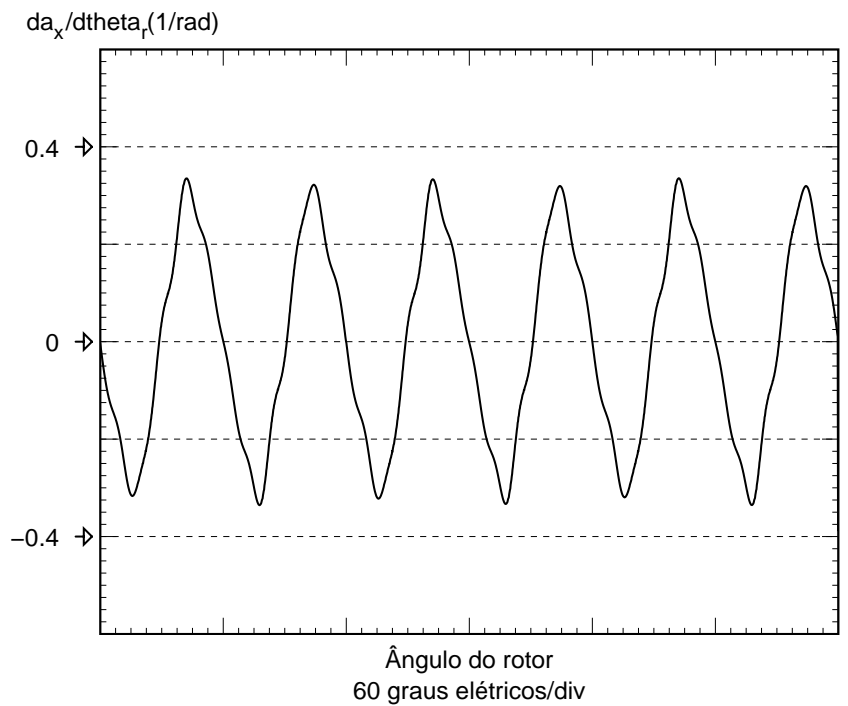

Figura 4.5: Parâmetro $\frac{d a_{x}}{d \theta_{r}}$ para a máquina utilizada, em função da posição rotórica $\left(\theta_{r}\right)$. 


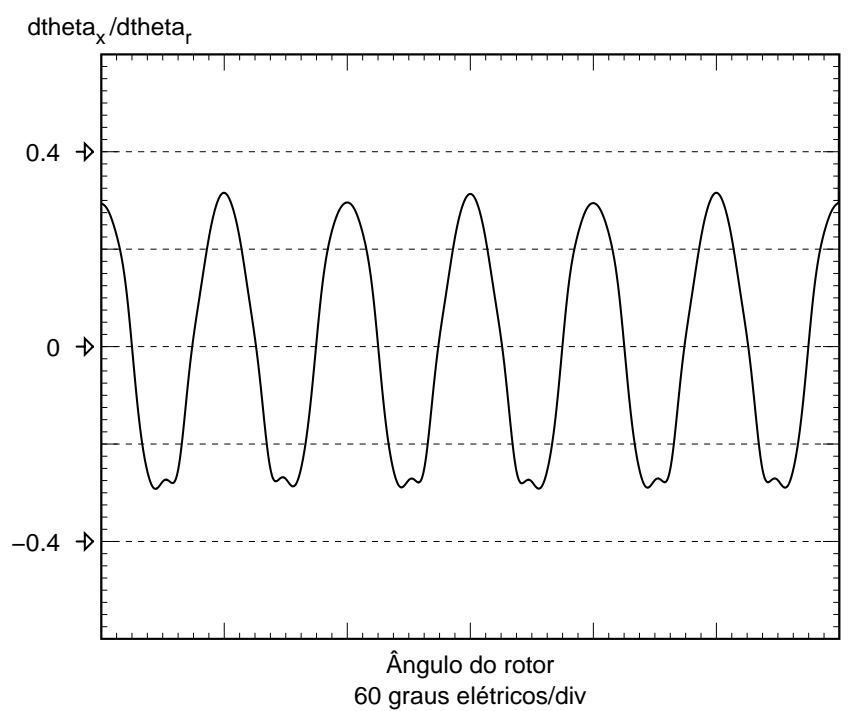

Figura 4.6: Parâmetro $\frac{d \theta_{x}}{d \theta_{r}}$ para a máquina utilizada, em função da posição rotórica $\left(\theta_{r}\right)$.

Tabela 4.1: Parâmetros mecânicos utilizados na simulação.

\begin{tabular}{|l|r|r|l|}
\hline Descrição & Símbolo & Valor & Unidade \\
\hline Momento de inércia do rotor da máquina & $J_{m}$ & $4,2 \cdot 10^{-3}$ & $\mathrm{~kg} \cdot \mathrm{m}^{2}$ \\
\hline Momento de inércia da carga & $J_{c}$ & 0 & $\mathrm{~kg} \cdot \mathrm{m}^{2}$ \\
\hline Constante de atrito viscoso da máquina & $B_{m}$ & $3,032 \cdot 10^{-3}$ & $\mathrm{~kg} \cdot \mathrm{m}^{2} / \mathrm{s}$ \\
\hline Constante de atrito viscoso da carga & $B_{c}$ & 0 & $\mathrm{~kg} \cdot \mathrm{m}^{2} / \mathrm{s}$ \\
\hline Torque de carga & $T_{c}$ & -2 & $\mathrm{~N} \cdot \mathrm{m}$ \\
\hline
\end{tabular}

$J_{c}$ : momento de inércia da carga;

$B_{m}$ : constante de atrito viscoso da máquina;

$B_{c}$ : constante de atrito viscoso da carga;

$T_{c}$ : torque de carga.

\subsection{Simulação em Malha Fechada de Corrente}

As simulações foram realizadas diretamente em linguagem $\mathrm{C}^{1}$, utilizando algoritmo "Runge-Kutta" de quinta ordem. Alguns cálculos também foram realizados utilizando-

\footnotetext{
${ }^{1}$ Utilizou-se o compilador GCC (GNU C Compiler) http://www.gnu.org.
} 
se o software GNU Octave ${ }^{2}$, que também foi utilizado para a geração dos gráficos. A utilização direta da linguagem $\mathrm{C}$, sem o uso de um software específico de simulação, possibilitou uma alta velocidade de cálculo, resultando em um tempo gasto com as simulações bem menor.

O esquema de controle utilizado na simulação em malha fechada é visto na figura 4.7. Em termos da simulação realizada, o bloco do motor representa as equações por fase da máquina (equações equações 3.1 e 3.2) e a equação mecânica (equação 4.1). Os blocos $T, c_{x}, e^{j \theta_{r}}$ (e seus inversos) e $z_{p} \sqrt{\frac{3}{2}} \Phi_{m}$ tratam do modelo vetorial da máquina. Portanto, para a simulação, as tensões $v_{a}, v_{b}$ e $v_{c}$ são determinadas através do modelo vetorial da MSIP e aplicadas ao modelo por fase.

Os blocos $G_{d}, G_{q}, G_{i q x}$ e $G_{v_{d x}}$ são os blocos de controle. Seus valores foram determinados através dos melhores resultados obtidos pela simulação. Os valores utilizados são:

$$
\begin{aligned}
& G_{d}=0 \\
& G_{q}=500+\frac{5 \cdot 10^{3}}{s}+200 s \\
& G_{i q x}=-0.3 \\
& G_{v_{d x}}=50
\end{aligned}
$$

Considerando-se as equações e algoritmo de simulação, a figura 4.8 mostra os blocos de equações utilizados no programa de simulação. As transformações e equações vetoriais e de controle calculam os ciclos de trabalho (duty cycles $-\delta$ ) que são aplicados ao bloco de PWM. Além do cálculo dos ciclos de trabalho empregar as equações vetoriais desenvolvidas aqui, utiliza-se uma forma de onda não senoidal para a determinação desses ciclos de trabalho. Após o cálculo do ângulo e da amplitude da tensão $v_{d q_{x}}$ a ser aplicada ao estator, utilizam-se as equações 4.3 a 4.7. Utiliza-se a equação 4.4, ao invés de se utilizar a função seno, para que diminua-se o número de chaveamentos, devido ao PWM, e para que a tensão aplicada à máquina possua amplitude maior do que amplitude conseguida com a utilização da função seno (Trzynadlowski et al., 1997).

\footnotetext{
${ }^{2}$ GNU Octave http://www.octave.org.
} 


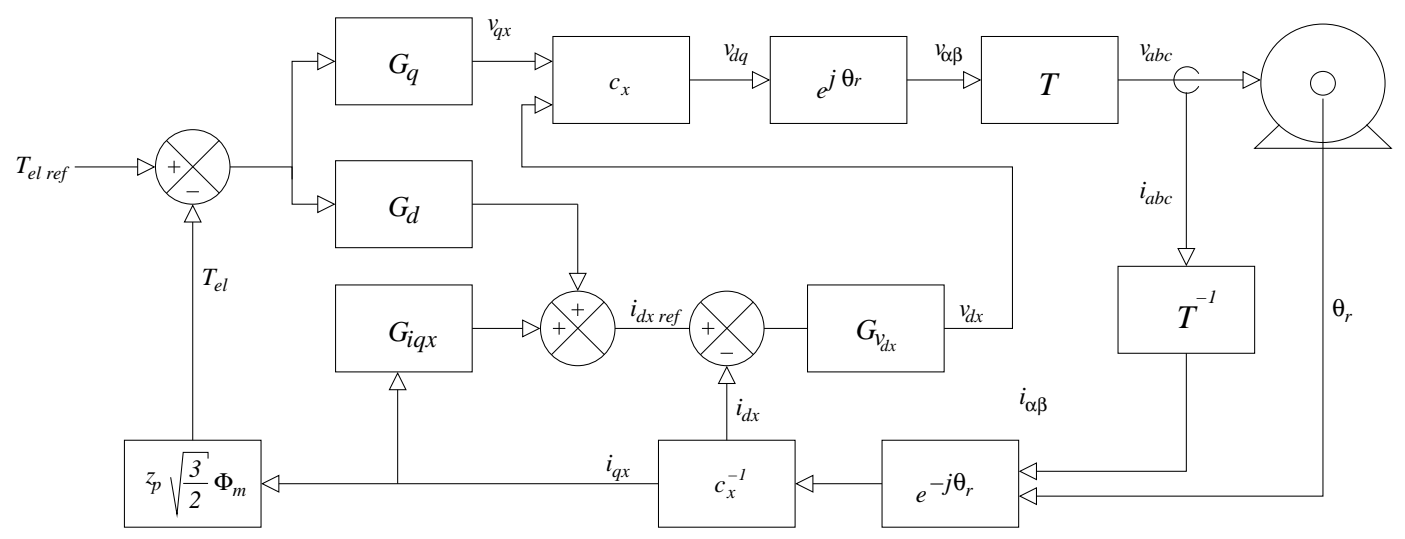

Figura 4.7: Diagrama de controle utilizado na simulação da máquina em malha fechada de corrente.

$$
\begin{gathered}
v_{d q_{x}}=V_{v} e^{j \theta_{v}} \\
\operatorname{pwmdiss}(M, \theta)= \begin{cases}M \cos \left(\theta-30^{\circ}\right) & p / 0^{\circ} \leq \theta<120^{\circ} \\
0 & p / 120^{\circ} \leq \theta<240^{\circ} \\
M \cos \left(\theta+30^{\circ}\right) & p / 240^{\circ} \leq \theta<360^{\circ}\end{cases} \\
M_{v}=\sqrt{2} \frac{V_{v}}{V_{B U S}} \\
\gamma=\theta_{r}+\theta_{x}+\theta_{v}+90^{\circ} \\
\delta_{a}=\operatorname{pwmdiss}\left(M_{v}, \gamma\right) \\
\delta_{b}=\operatorname{pwmdiss}\left(M_{v}, \gamma-120^{\circ}\right) \\
\delta_{c}=\operatorname{pwmdiss}\left(M_{v}, \gamma+120^{\circ}\right)
\end{gathered}
$$

O bloco de PWM determina as tensões que são aplicadas às equações diferenciais elétricas do modelo por fase $\left(v_{a}, v_{b}\right.$ e $\left.v_{c}\right)$ com base nos ciclos de trabalho de cada fase do estator. Das equações diferenciais elétricas, obtém-se as correntes nas três fases da máquina, que são utilizadas para o cálculo do torque eletromagnético. O torque 


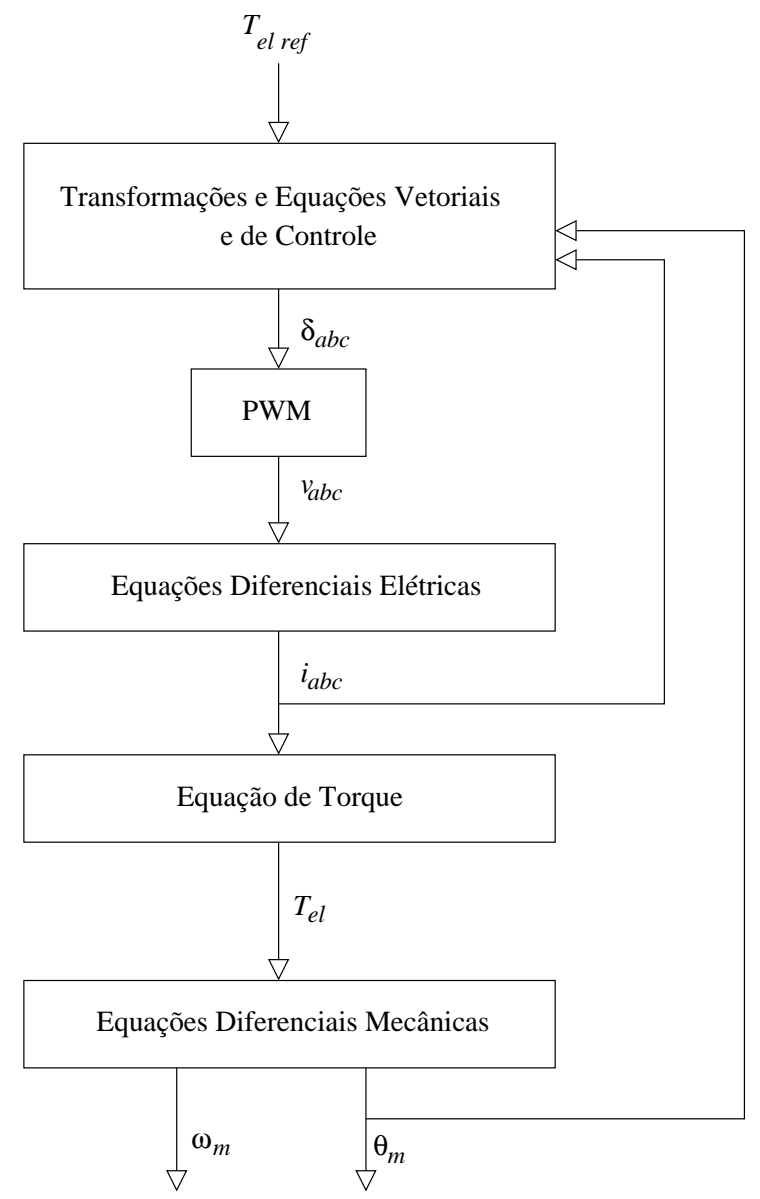

Figura 4.8: Diagrama de controle utilizado na simulação da máquina em malha fechada de corrente.

eletromagnético é então aplicado às equações diferenciais mecânicas, resultando na velocidade e na posição rotóricas $\left(\omega_{m}\right.$ e $\left.\theta_{m}\right)$.

Nota-se que o torque eletromagnético, utilizado nas equações de controle, é calculado segundo o modelo vetorial da MSIP (equação 3.17) e, nas equações diferenciais do modelo da máquina, o torque eletromagnético é calculado segundo o modelo convencional por fase. Portanto, no caso de algum erro nas equações do modelo vetorial da MSIP, as simulações não mostrariam os resultados corretamente.

Na figura 4.9, é apresentada a corrente de estator e o torque eletromagnético da máquina acionada por um inversor com o modelo vetorial proposto. A máquina é acionada sem PWM. É aplicada uma referência de torque à máquina de $6 \mathrm{~N} \cdot \mathrm{m}$ de 0 a 0,05s. Após esse intervalo, aplica-se uma referência de $3 \mathrm{~N} \cdot \mathrm{m}$. Nas figuras 4.10 e 4.11 são aplicadas as mesmas referências de torque, no entanto, a tensão nas fases do estator 


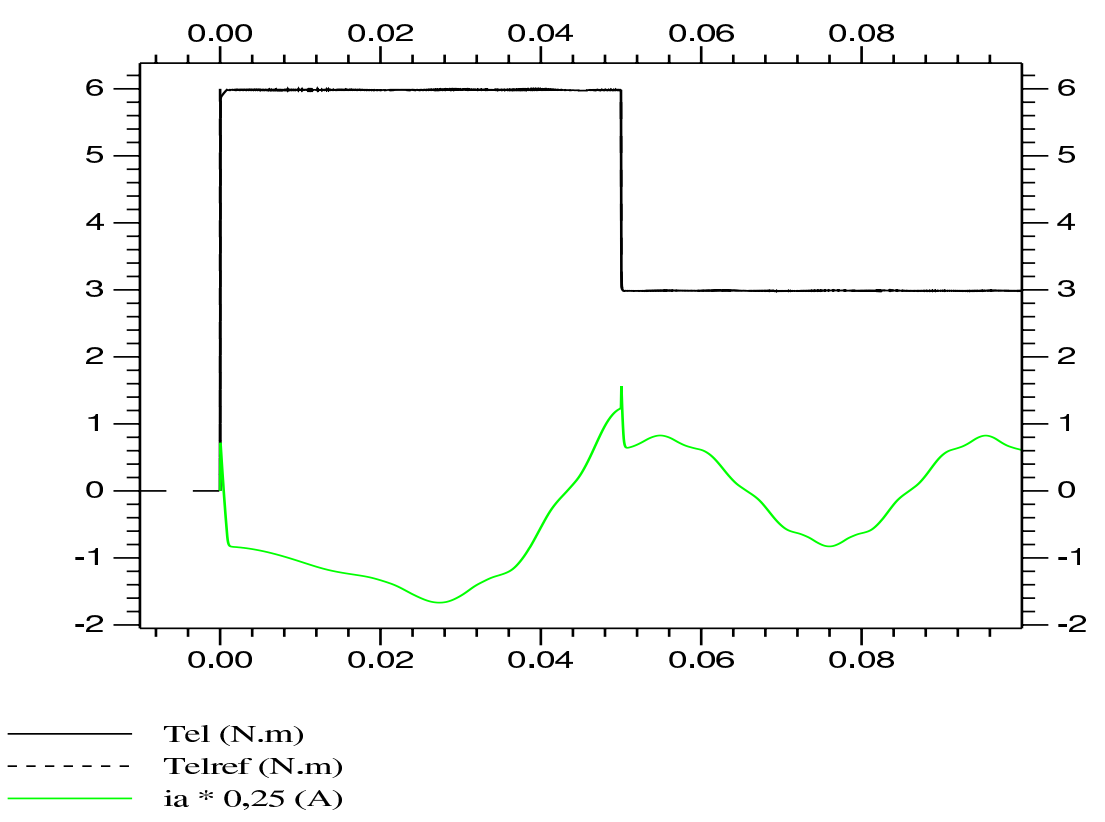

Figura 4.9: Torque de partida e corrente da máquina acionada com controle vetorial (sem utilização de PWM).

é aplicada por meio de PWM, com freqüências de 5,88 e $20 \mathrm{kHz}$, respectivamente.

A figura 4.12 mostra a aplicação de uma referência de torque na máquina. A referência de torque consiste em uma função seno com amplitude de $2,6 \mathrm{~N} \cdot \mathrm{m}$ e com período de 2s. Nessa simulação, as tensões aplicadas ao estator da máquina são tensões contínuas $^{4}$, sem o uso de PWM. A malha de controle é fechada por um controlador PI.

Nota-se, na figura, que após aproximadamente 1,5s o torque eletromagnético da máquina não acompanha mais o torque de referência da máquina. Isso ocorre devido à velocidade do rotor ser alta, gerando uma FEM alta interna. Esse fato faz com que a tensão aplicada nas bobinas das fases do estator fique baixa, insuficiente para fazer com que a taxa de variação da corrente de estator seja suficiente para acompanhar a referência aplicada. Embora a simulação mostre uma degradação no desempenho da máquina, isso não deve ocorrer na prática, pois a velocidade em que isso ocorre é quase 2 vezes maior que a velocidade nominal da máquina. Máquinas com ímã permanente na superfície do motor não devem operar em altas velocidades com perigo de danos

\footnotetext{
${ }^{3}$ Esse valor foi escolhido devido ao circuito de controle utilizar essa freqüência, conforme mostrado no capítulo 5 .

${ }^{4}$ Tensão contínua no sentido de não ser descontínua ou chaveada, não tendo nada a ver com não ser tensão alternada.
} 


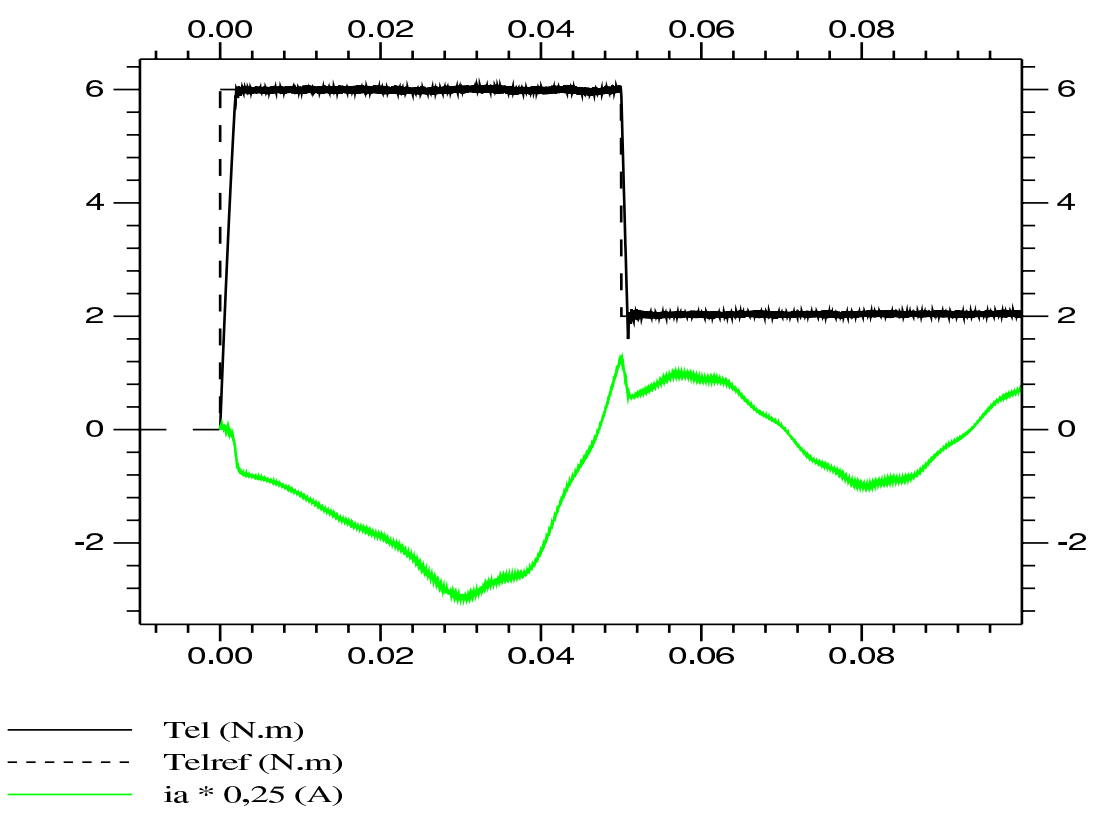

Figura 4.10: Torque de partida e corrente da máquina acionada com controle vetorial, com $f_{P W M}=5,88 \mathrm{kHz}$.

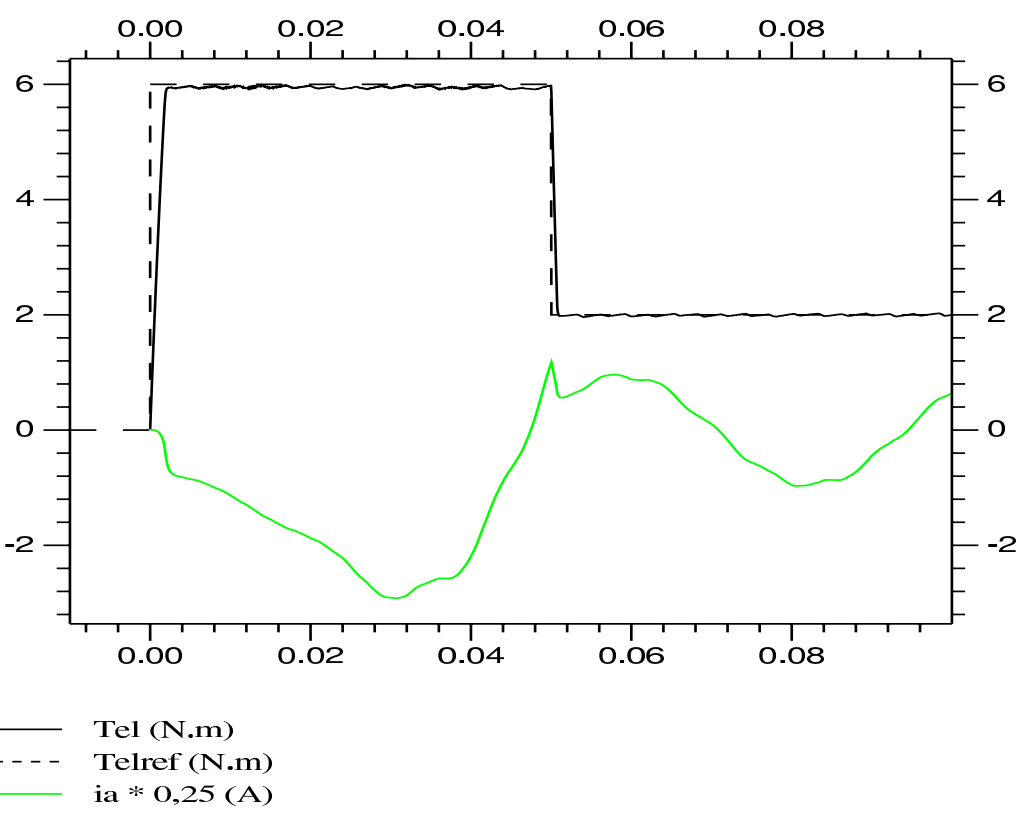

Figura 4.11: Torque de partida e corrente da máquina acionada com controle vetorial, com $f_{P W M}=20 \mathrm{kHz}$. 


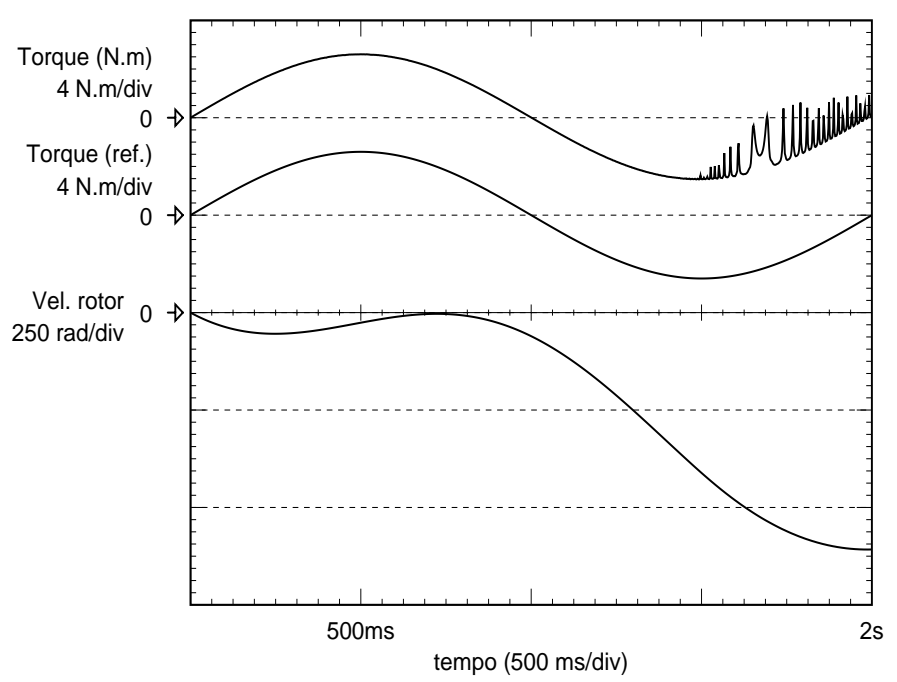

Figura 4.12: MSIP seguindo referência de torque.

permanentes à máquina.

É importante notar que a máquina apresenta uma aceleração negativa de velocidade devido à aplicação de um torque de carga, contrário ao sentido positivo de movimento, de $2 \mathrm{~N} \cdot \mathrm{m}$. Esse torque de carga é considerado em todas as simulações realizadas. Não são consideradas carga inercial e nem carga de atrito, pois esses dois tipos de carga tendem a suavizar possíveis ondulações do sistema mecânico. Como o objetivo é a avaliação das ondulações nas grandezas mecânicas, como torque, velocidade do rotor e posição do rotor, as simulações são realizadas considerando-se o pior caso (sem atrito, apenas o do eixo do próprio motor, e sem carga inercial).

A figura 4.13 apresenta a MSIP seguindo uma referência de velocidade. A malha de controle é fechada por controlador PI. A referência de velocidade é uma função seno com período de $2 \mathrm{~s}$ e amplitude de $2000 \mathrm{rpm}$, que é a velocidade nominal da máquina. A posição do rotor atinge um ponto máximo de 133,25 radianos, ou seja, 21,18 voltas. Pode-se notar pelo gráfico que a posição do rotor não possui ondulações, bem como a velocidade e o torque eletromagnético desenvolvido pela máquina.

Na figura 4.13, a máquina está parada no instante 0 e não possui tensões aplicadas. Existe inicialmente um pequeno transitório de torque eletromagnético no instante 0 , que devido à escala do gráfico, não é mostrado. Esse transitório pode ser melhor visualizado 


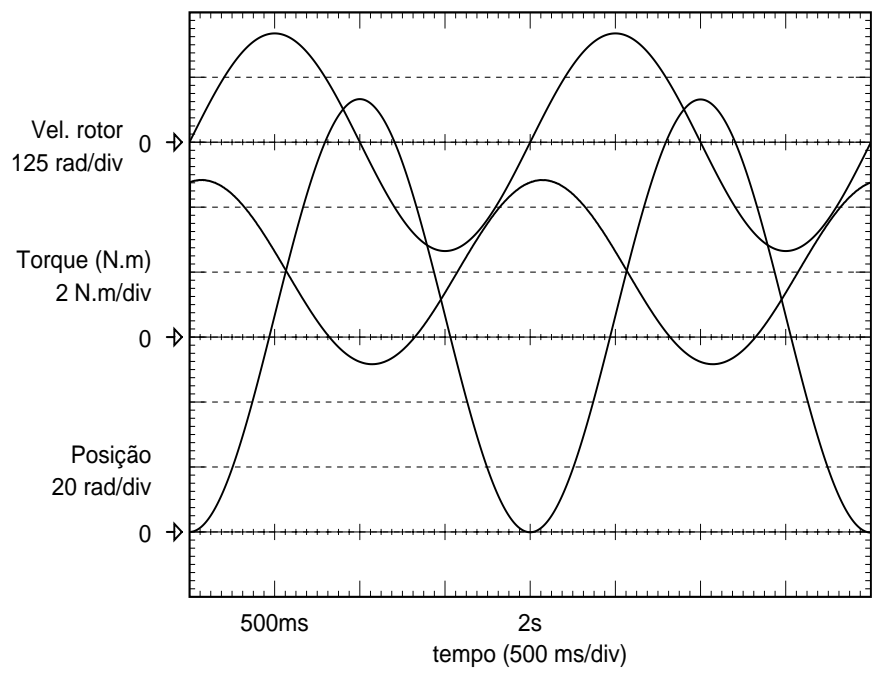

Figura 4.13: MSIP seguindo referência de velocidade.

na figura 4.14 .

A figura 4.15 apresenta a MSIP seguindo uma referência de posição. A malha de controle é fechada por controlador PI. Nessa simulação, a referência de posição é uma função seno com amplitude de 188,50 radianos, o que equivale a 30 voltas do rotor. O período da função é de 4 segundos. A referência de posição está mostrada na figura por "ref.". A posição da máquina está livre de ondulações, bem como o torque eletromagnético e a velocidade em regime, após $0,5 \mathrm{~s}$.

Nessa simulação, o torque eletromagnético da máquina foi limitado em $9 \mathrm{~N} \cdot \mathrm{m}$. A velocidade da máquina ultrapassou a velocidade nominal, o que indica que essa situação está além das possibilidades práticas, mas serve bem como exemplo de controle de torque eletromagnético. Na prática, além de um limitador de torque eletromagnético e de correntes de estator, é necessário também um limitador de velocidade. Esse limitador não foi considerado por tratar-se de uma simulação e por se desejar mostrar a máquina seguindo uma trajetória de posição que explore e garanta a eficácia do modelo. 


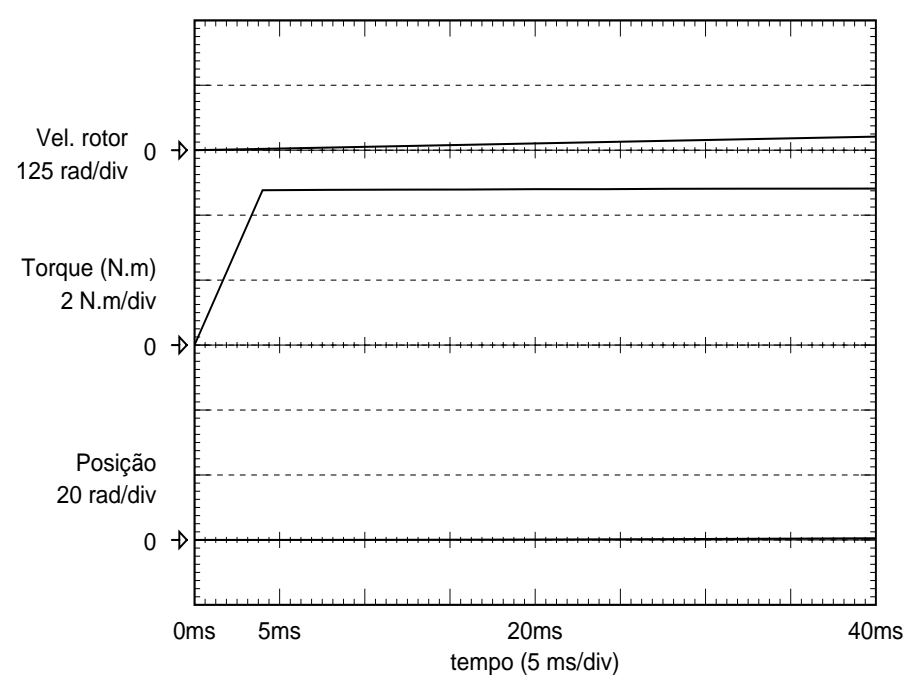

Figura 4.14: MSIP seguindo referência de velocidade - detalhe do transitório inicial.

\subsection{Simulação em Malha Aberta de Corrente}

Na figura 4.16 é apresentado o esquema utilizado para a simulação em malha aberta de corrente. Através da figura, nota-se que somente o valor da posição rotórica $\left(\theta_{r}\right)$ é utilizado nos cálculos das tensões a serem aplicadas ao estator da máquina $\left(v_{a}, v_{b} \mathrm{e}\right.$ $\left.v_{c}\right)$.

O bloco "Equações de Regime Permanente" refere-se às equações de regime permanente da MSIP, apresentadas no capítulo 3, equações 3.24 e 3.25.

No caso da simulação em malha fechada de corrente, é necessário que se conheçam $\Phi_{m}$ e as formas de onda de FEM. Para o acionamento da máquina em malha aberta de corrente, deve-se conhecer todos os parâmetros da MSIP, além dos citados anteriormente, deve se conhecer $R_{s}, L_{s}$ e $M_{s}$.

Na figura 4.17, é apresentada uma simulação da MSIP acionada em malha aberta de corrente, sem a utilização de PWM na tensão aplicada ao estator.

Na figura 4.18 a máquina é acionada em malha aberta de corrente, com freqüência de PWM de 5,88kHz. Pode-se notar que a maior diferença entre o gráfico da figura 4.18 e o da figura 4.10, é o transitório do torque eletromagnético que, no caso da malha aberta de corrente, é bem mais lento. 


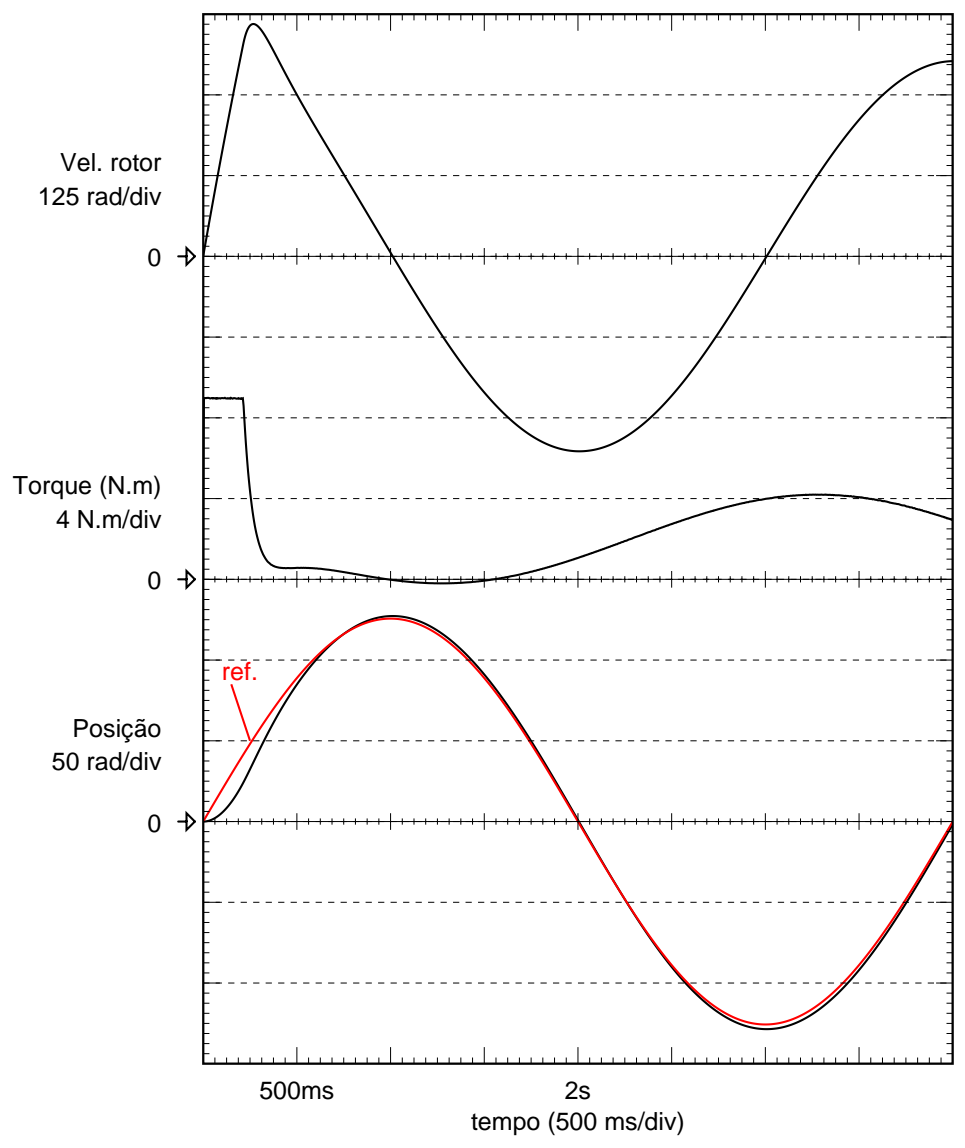

Figura 4.15: MSIP seguindo referência de posição.

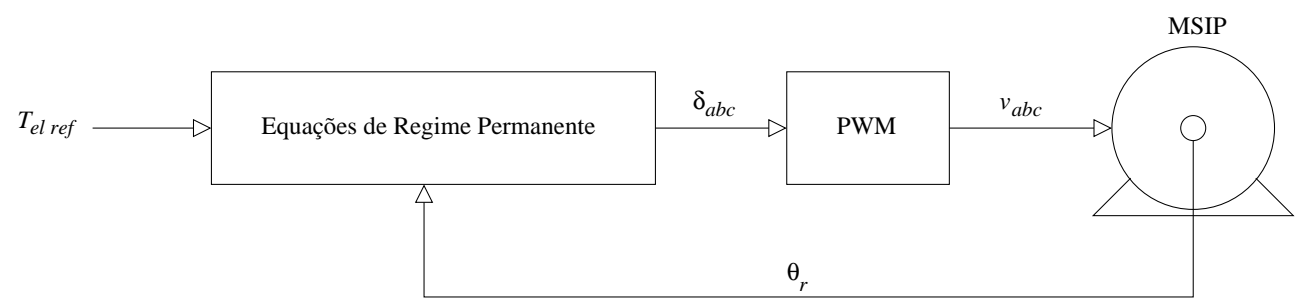

Figura 4.16: Esquema da simulação em malha aberta de corrente. 

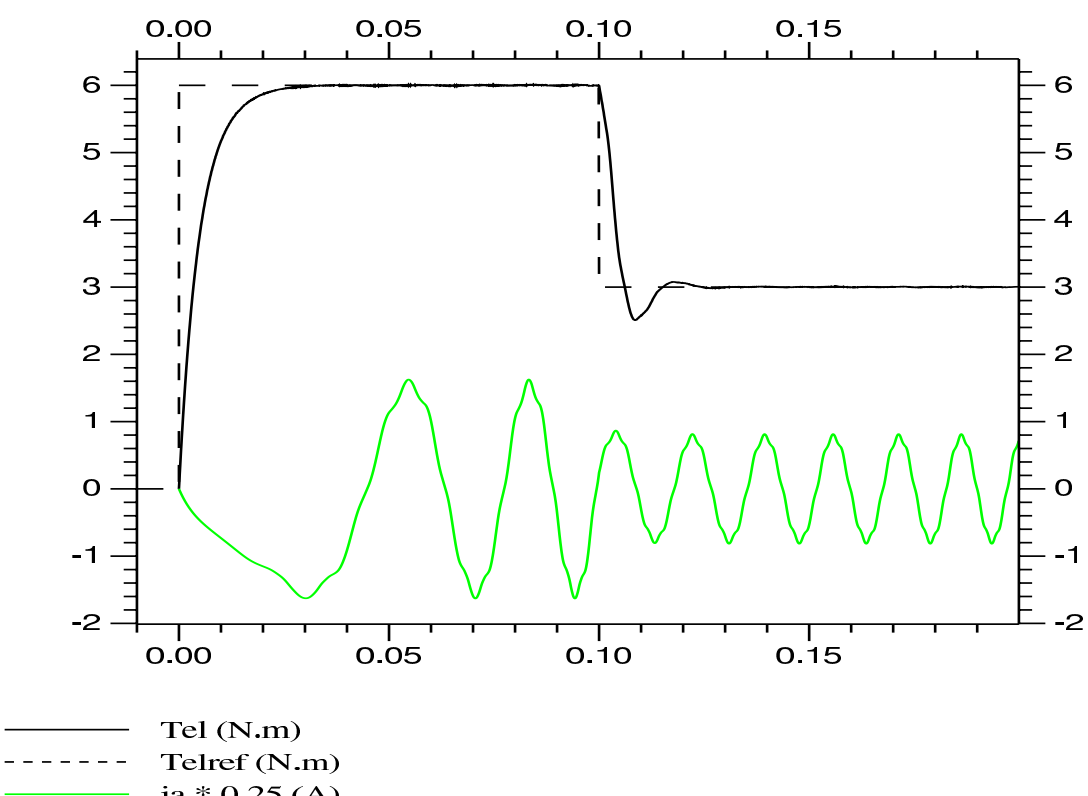

Figura 4.17: Simulação em malha aberta de corrente, sem a utilização de PWM nas tensões aplicadas ao estator.

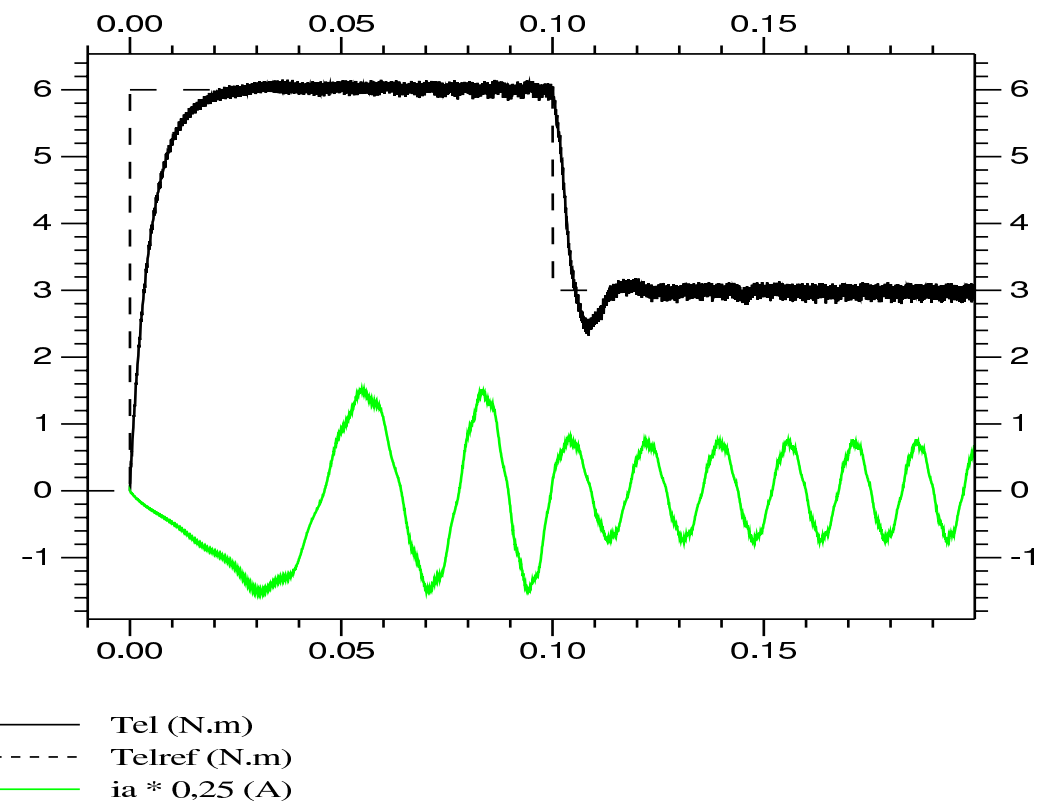

Figura 4.18: Simulação em malha aberta de corrente, com $f_{P W M}=5,88 \mathrm{kHz}$. 


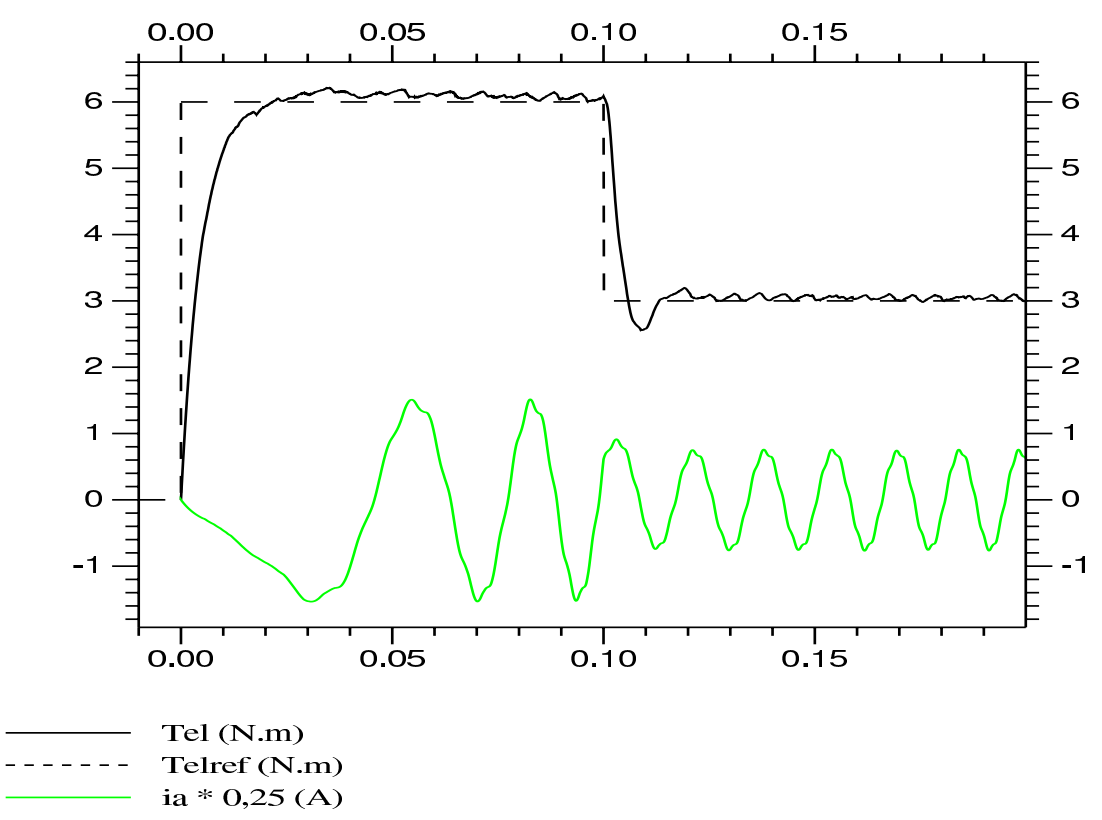

Figura 4.19: Simulação em malha aberta de corrente, com $f_{P W M}=20 \mathrm{kHz}$.

As ondulações presentes no torque eletromagnético da figura 4.18 são devido ao PWM de 5,88kHz. Essas ondulações de alta freqüência são filtradas pelas constantes mecânicas do sistema, não causando deterioração em sistemas de posicionamento.

Na figura 4.19 é apresentada a mesma simulação para uma freqüência de PWM de $20 \mathrm{kHz}$.

\subsection{Simulação no Modo Seis-Pulsos}

As figuras 4.20 e 4.21 mostram a velocidade do rotor e o torque eletromagnético da máquina na partida, acionada por um inversor com controle clássico "seis-pulsos", para diferentes freqüências de PWM. Como no caso anterior, o motor é acionado com a malha de corrente aberta. Esses gráficos mostram que a máquina com inversor "seispulsos" apresenta grandes ondulações no torque eletromagnético. Isso ocorre devido a sua forma de onda de tensão induzida não ser exatamente trapezoidal e também à comutação das fases do estator, a cada 60 graus elétricos, não ser ideal.

A simulação no modo seis-pulsos serve para mostrar que sem um controle apropriado, a tendência da máquina é apresentar altas ondulações no torque eletromagnético, cerca de 25 a $30 \%$ na prática (Holtz \& Springob, 1996). No caso de uma máquina 


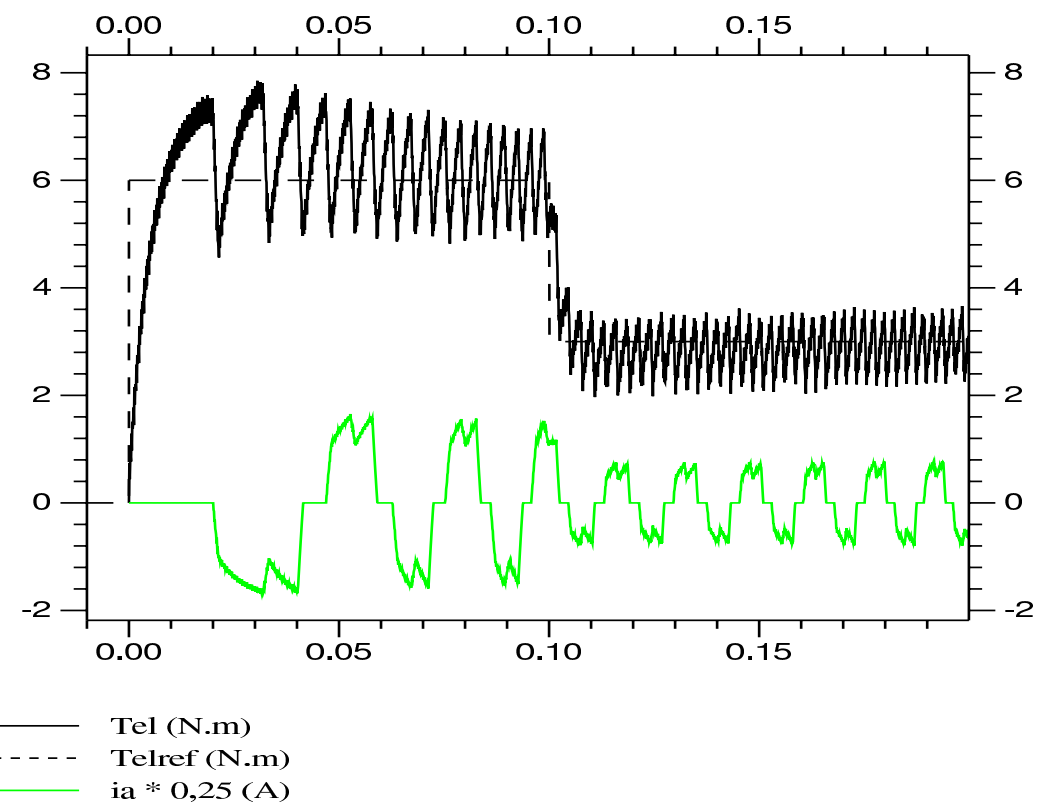

Figura 4.20: Torque de partida e corrente da máquina acionada com inversor "seispulsos" $\left(f_{P W M}=5,88 \mathrm{kHz}\right)$.

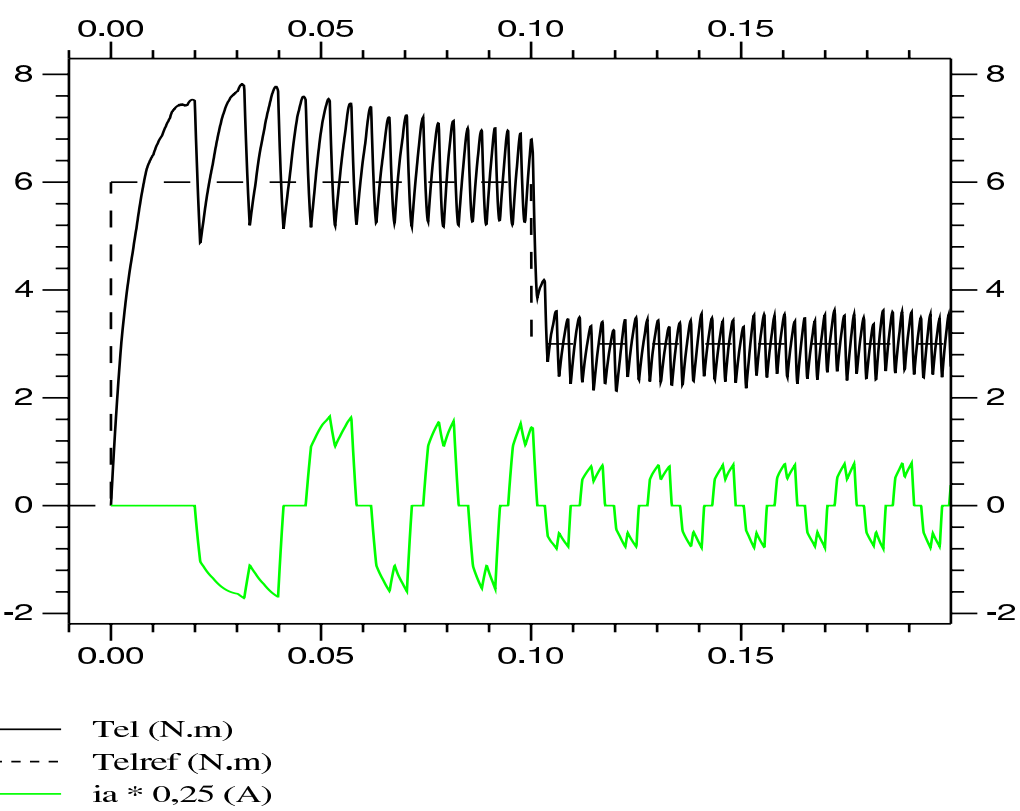

Figura 4.21: Torque de partida e corrente da máquina acionada com inversor "seispulsos" $\left(f_{P W M}=20 \mathrm{kHz}\right)$. 
senoidal alimentada com um inversor com PWM senoidal essas ondulações no torque eletromagnético seriam bem menores (Holtz \& Springob, 1996). O controle vetorial proposto permite uma "compensação" pela forma de onda da máquina não ser senoidal, resultando em um decréscimo considerável nas ondulações no torque eletromagnético causadas por essa forma de onda não senoidal. 


\section{Capítulo 5}

\section{Implementação do Controle Vetorial}

Nesse capítulo, serão apresentados alguns resultados práticos obtidos com a implementação do controle vetorial proposto. Alguns gráficos da corrente real de estator são mostrados confrontando-se com seus equivalentes simulados. O torque eletromagnético é então calculado através das correntes nas fases do estator. Em outro experimento, a posição do rotor da máquina é medida com a aplicação de uma carga constante no eixo, considerando dois casos de alimentação da máquina: através de conversor no modo "seis-pulsos" e com controle vetorial não senoidal em malha aberta de corrente. Com isso, pode-se verificar indiretamente as ondulações no torque eletromagnético.

Detalhes do hardware utilizado para o acionamento da máquina estão apresentados no apêndice $\mathrm{F}$; no apêndice $\mathrm{G}$, descreve-se o funcionamento do programa de controle utilizado na placa de controle.

\subsection{Implementação em Malha Aberta de Corrente}

A máquina foi acionada com controle vetorial em malha aberta de corrente. As tensões aplicadas ao estator da máquina foram calculadas com as equações apresentadas no capítulo 3 , as equações $3.23,3.24$ e 3.25 . Fixando-se um torque de referência, as tensões a serem aplicadas ao estator são calculadas em cada posição do rotor.

As figuras 5.1 a 5.7 apresentam alguns resultados de corrente de fase da máquina 
obtidos por simulação e através da medida no sistema físico. As correntes de fase de estator foram medidas pela tensão em um resistor de $0,47 \Omega$.

Em cada figura, são apresentados dois gráficos da corrente de fase em condições muito próximas de operação, mas um obtido através da corrente na máquina real (b) e outro pela simulação (a). Nesse último, é apresentado também o torque eletromagnético gerado pela máquina, calculado pela equação do torque eletromagnético do modelo por fase, equação 3.2 .

Pode-se notar que em todas as condições de operação apresentadas, a forma de onda de corrente obtida pela simulação é bem próxima à lida na máquina.

Tanto na simulação quanto na medida real, aplicou-se uma referência de torque ao sistema e aumentou-se ou diminuiu-se a carga de atrito a fim de se variar a velocidade de operação.

O programa de simulação levou em conta a maioria das limitações do circuito de controle empregado, tais como: atrasos na atualização dos ciclos de trabalho de PWM; valores utilizados internamente nos cálculos inteiros (ou valores discretizados; o processador utilizado não processa ponto flutuante, somente números inteiros - apêndice G); valores discretos (0 a 250) nos ciclos de trabalho de PWM; medida de velocidade e posição rotóricas apenas uma vez a cada ciclo de PWM (170 $\mathrm{Ps})$.

Exceto por pequenos detalhes, as figuras 5.1 a 5.7 mostram valores próximos entre as correntes obtidas por simulação e real. As diferenças ocorrem porque a máquina pode apresentar pequenas variações de seus parâmetros, como $R_{s}, L_{s}, M_{s}, \Phi_{m}$ ou mesmo $F_{\text {rabc }}$.

Um outro fator é a diferença entre o programa de simulação e o programa do circuito de controle, que possui tempos diferentes de leitura e atualização, embora tenha-se realizado um esforço para minimizar essas diferenças de tempos.

Os gráficos das figuras 5.6 e 5.7 mostram a operação da máquina para uma referência de torque menor que a dos outros gráficos. Devido à pequena escala de corrente, nota-se a grande componente de histerese na corrente e, conseqüentemente, no torque eletromagnético mostrado no gráfico de simulação (a). Essa histerese é devida à normalização dos valores, principalmente aos valores de ciclo de trabalho de PWM, e não à freqüência utilizada no PWM, como poderia-se supor. 


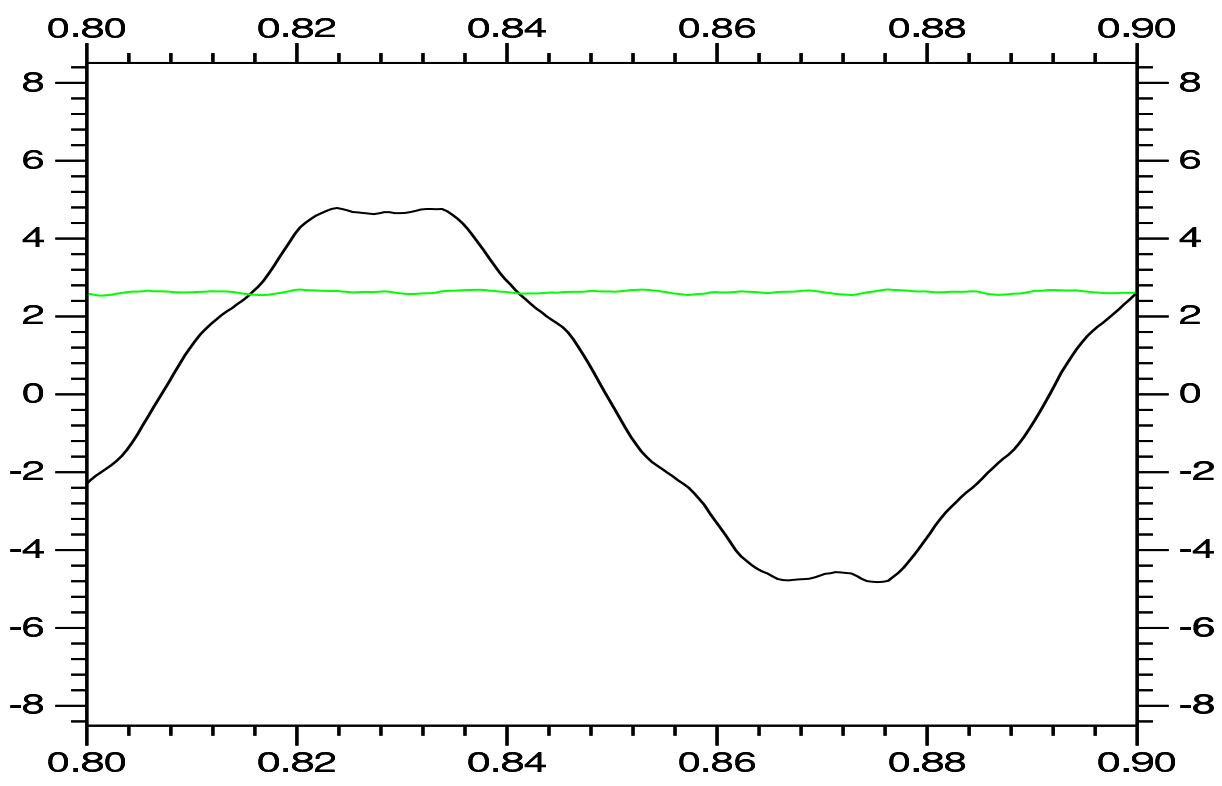

(a)

$\begin{array}{ll}\longleftarrow & \text { ia (A) } \\ \text { Tel (N.m) }\end{array}$

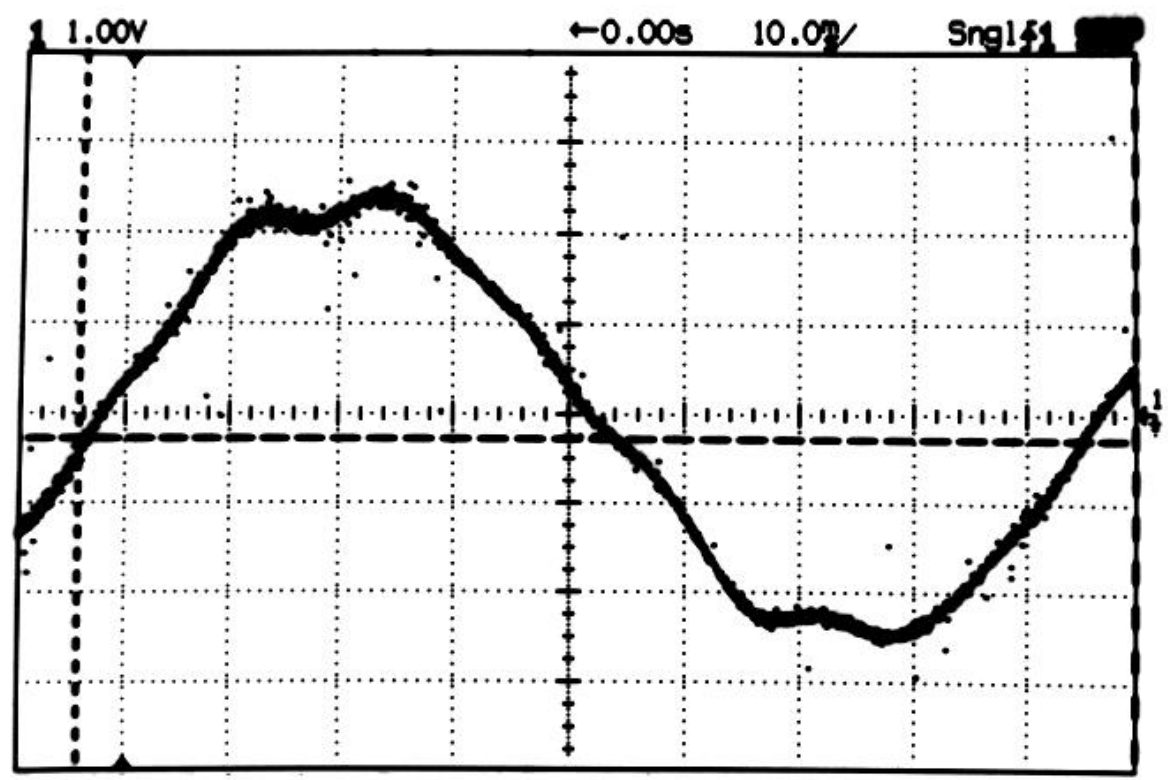

(b)

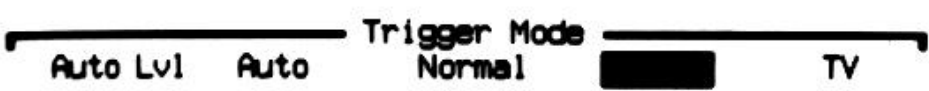

Figura 5.1: Simulação da corrente de fase e do torque eletromagnético (gráfico - a) e medida da corrente de fase (gráfico - b) na MSIP com controle vetorial em malha aberta de corrente (corrente: $2,13 \mathrm{~A} / \mathrm{div}, \omega_{m}=24,6 \mathrm{rad} / \mathrm{s}$ ). 


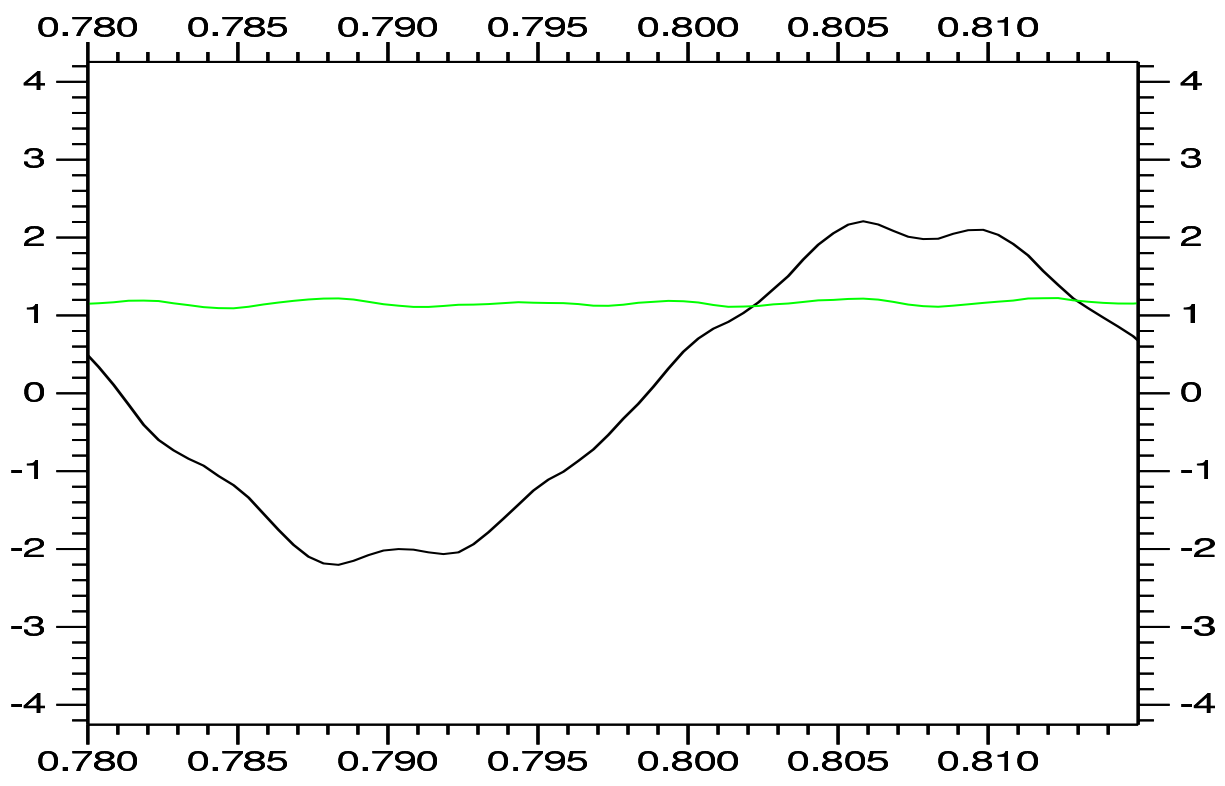

(a) $\longleftarrow \begin{aligned} & \text { ia (A) } \\ & \text { Tel (N.m) }\end{aligned}$

$\begin{array}{ll}\longleftarrow & \text { ia (A) } \\ \text { Tel (N.m) }\end{array}$

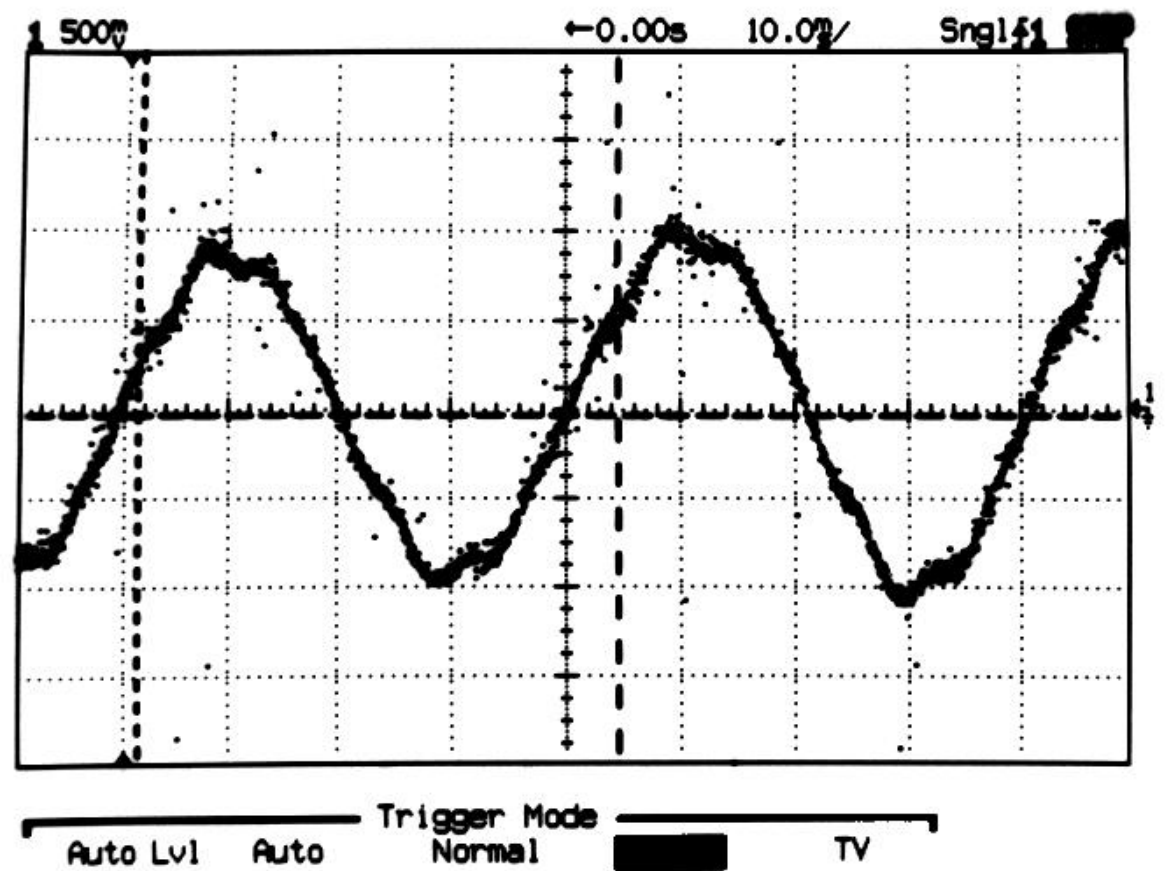

(b)

Figura 5.2: Simulação da corrente de fase e do torque eletromagnético (gráfico - a) e medida da corrente de fase (gráfico - b) na MSIP com controle vetorial em malha aberta de corrente (corrente: 1,06 A/div, $\omega_{m}=67,6 \mathrm{rad} / \mathrm{s}$ ). 


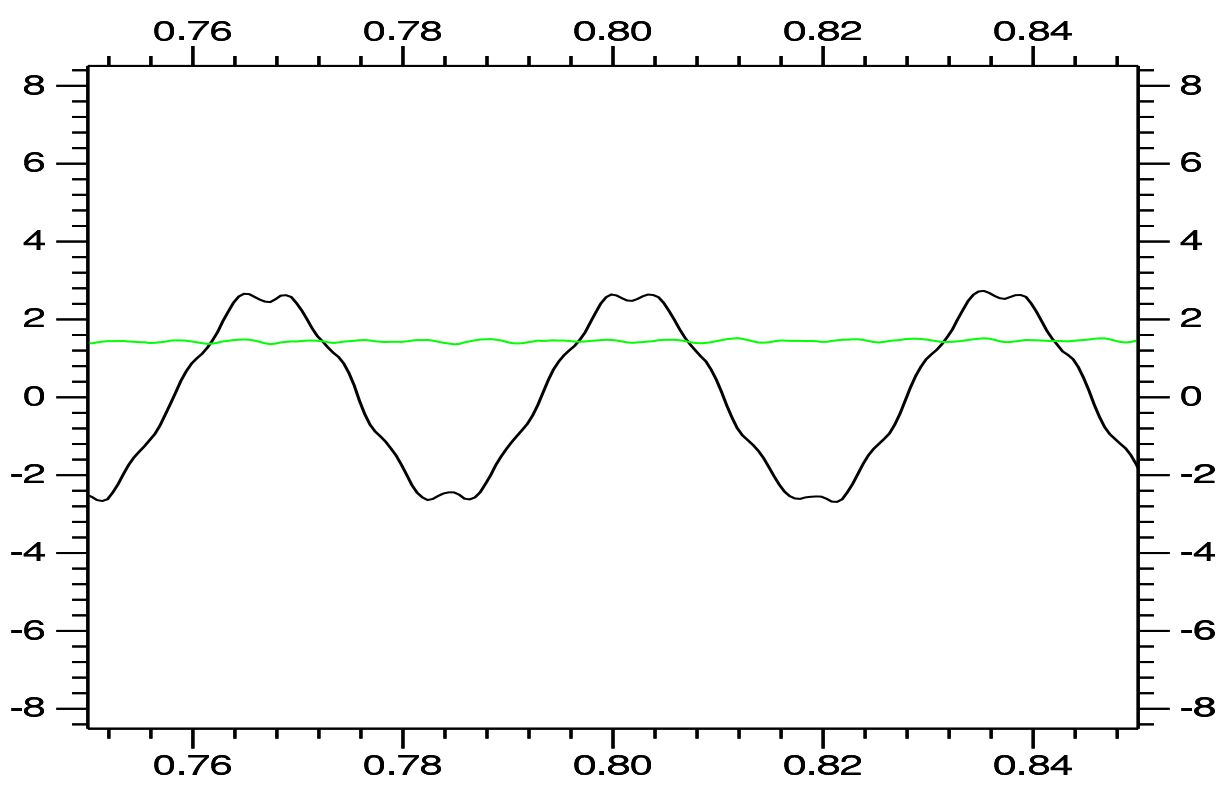

(a) $\longleftarrow \begin{aligned} & \text { ia (A) } \\ & \text { Tel (N.m) }\end{aligned}$

$\begin{array}{ll}\longleftarrow & \text { ia (A) } \\ \text { Tel (N.m) }\end{array}$

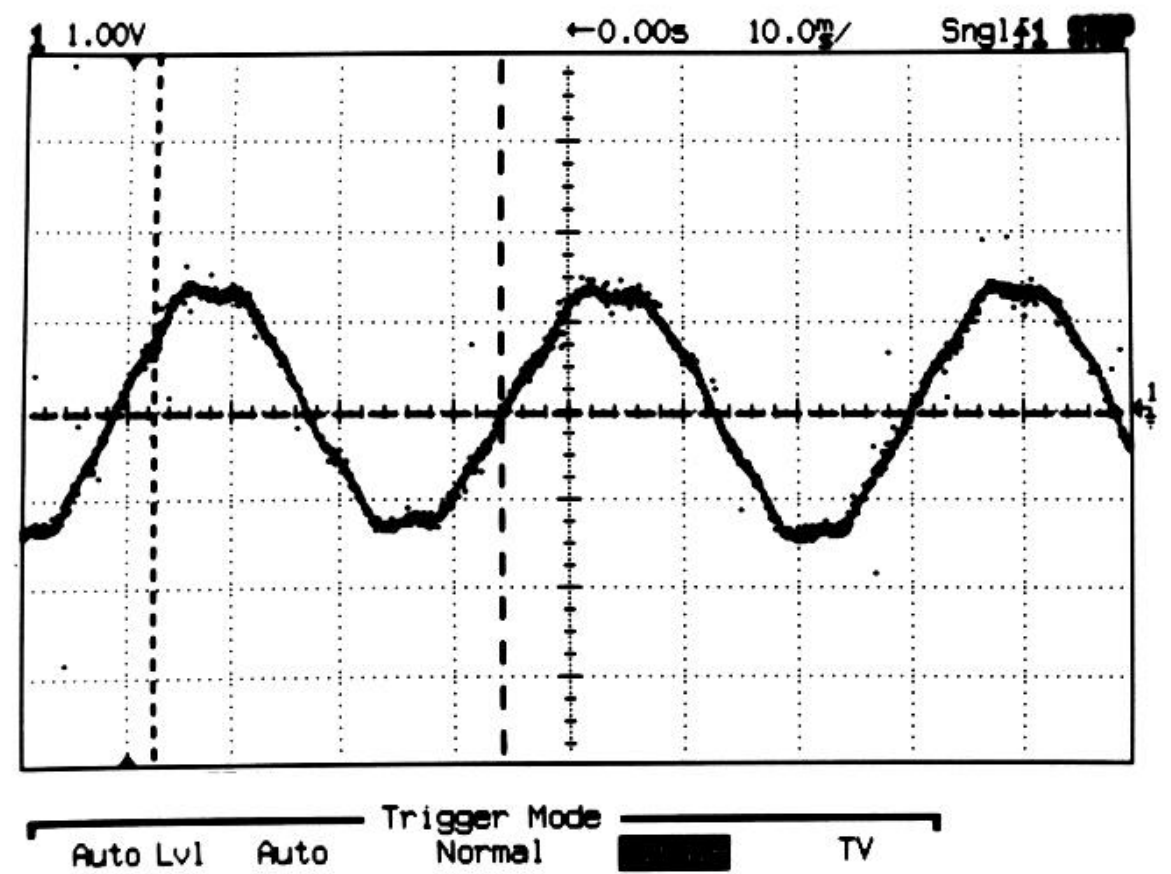

(b)

Figura 5.3: Simulação da corrente de fase e do torque eletromagnético (gráfico - a) e medida da corrente de fase (gráfico - b) na MSIP com controle vetorial em malha aberta de corrente (corrente: $2,13 \mathrm{~A} / \operatorname{div}, \omega_{m}=59,8 \mathrm{rad} / \mathrm{s}$ ). 


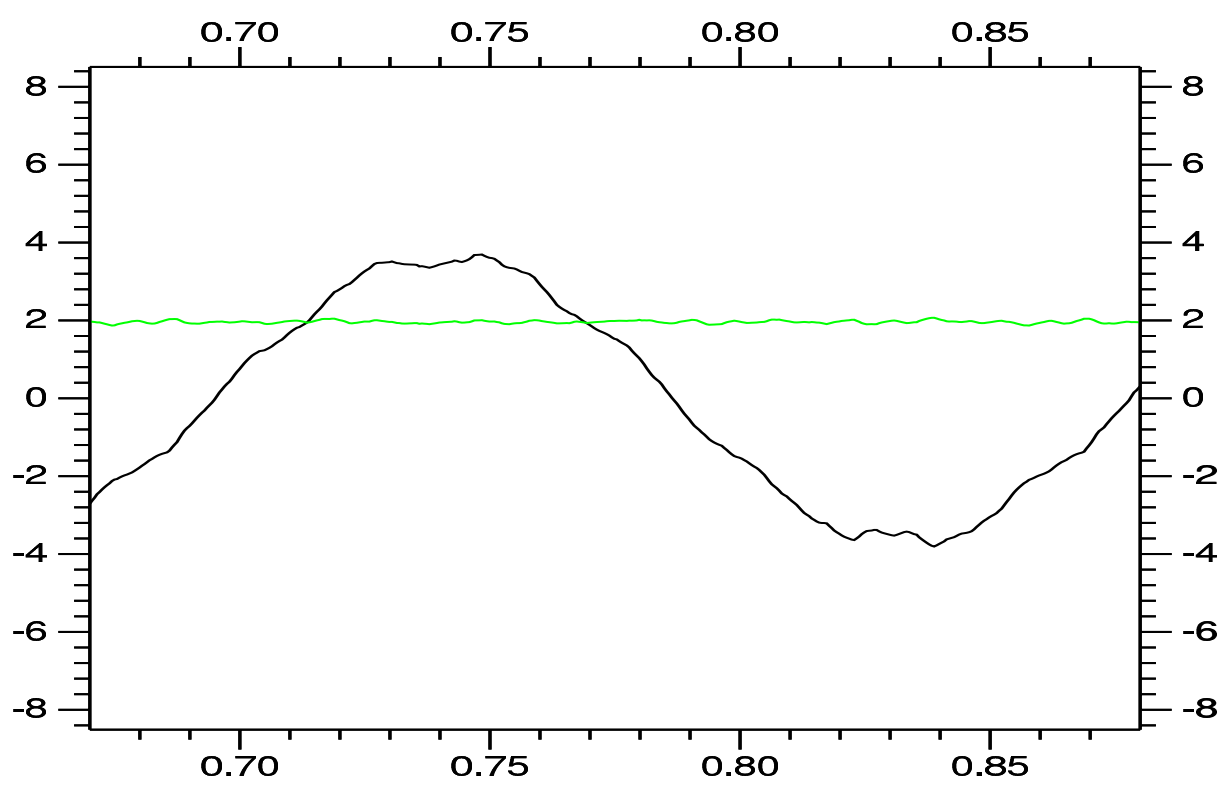

(a)

$\begin{array}{ll}\longleftarrow & \text { ia (A) } \\ \text { Tel (N.m) }\end{array}$

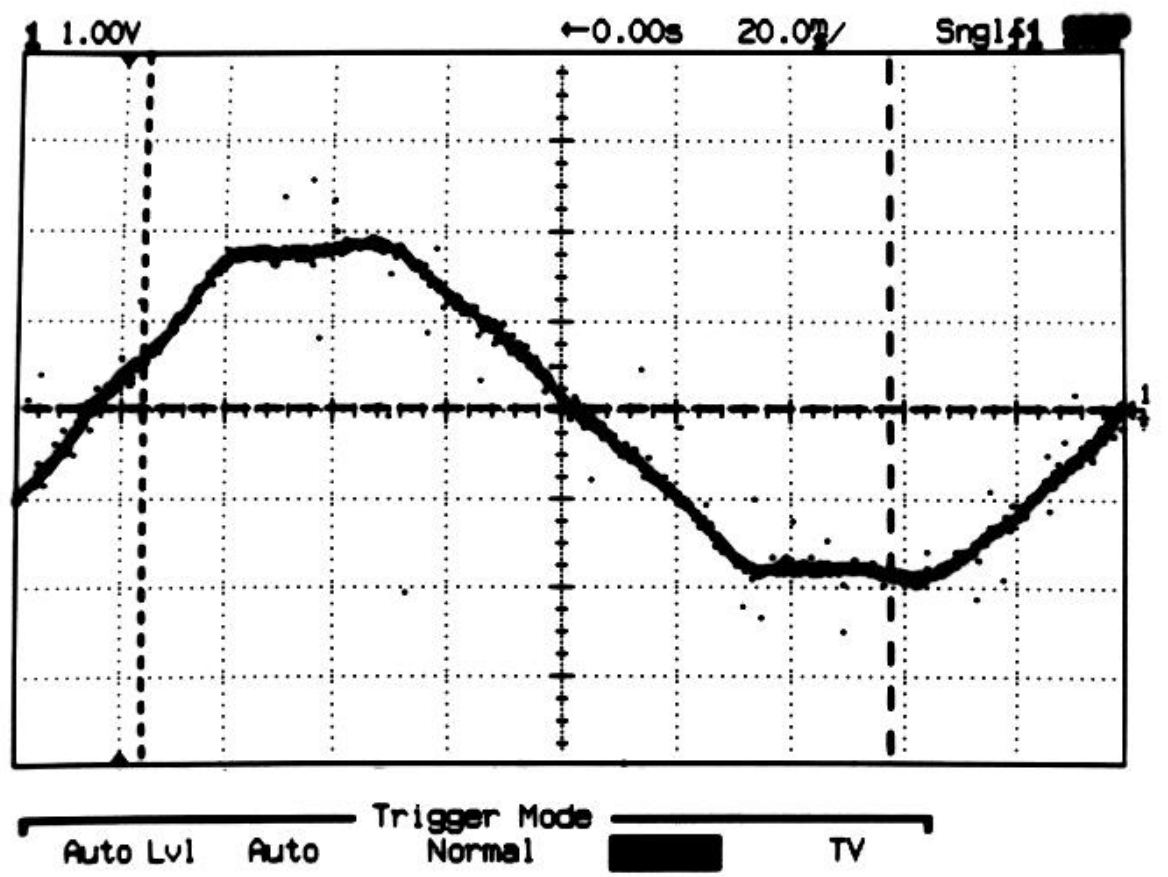

(b)

Figura 5.4: Simulação da corrente de fase e do torque eletromagnético (gráfico - a) e medida da corrente de fase (gráfico - b) na MSIP com controle vetorial em malha aberta de corrente (corrente: $2,13 \mathrm{~A} / \mathrm{div}, \omega_{m}=11,3 \mathrm{rad} / \mathrm{s}$ ). 


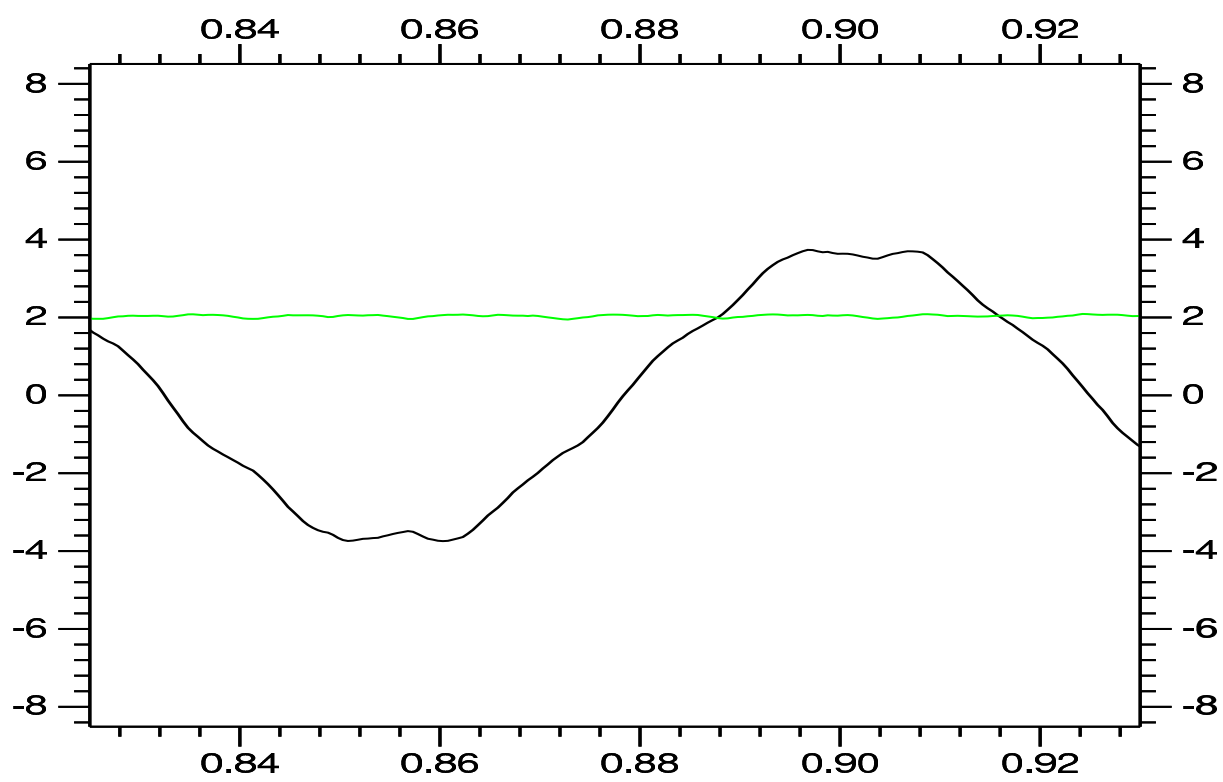

(a) ia (A)

Tel (N.m)

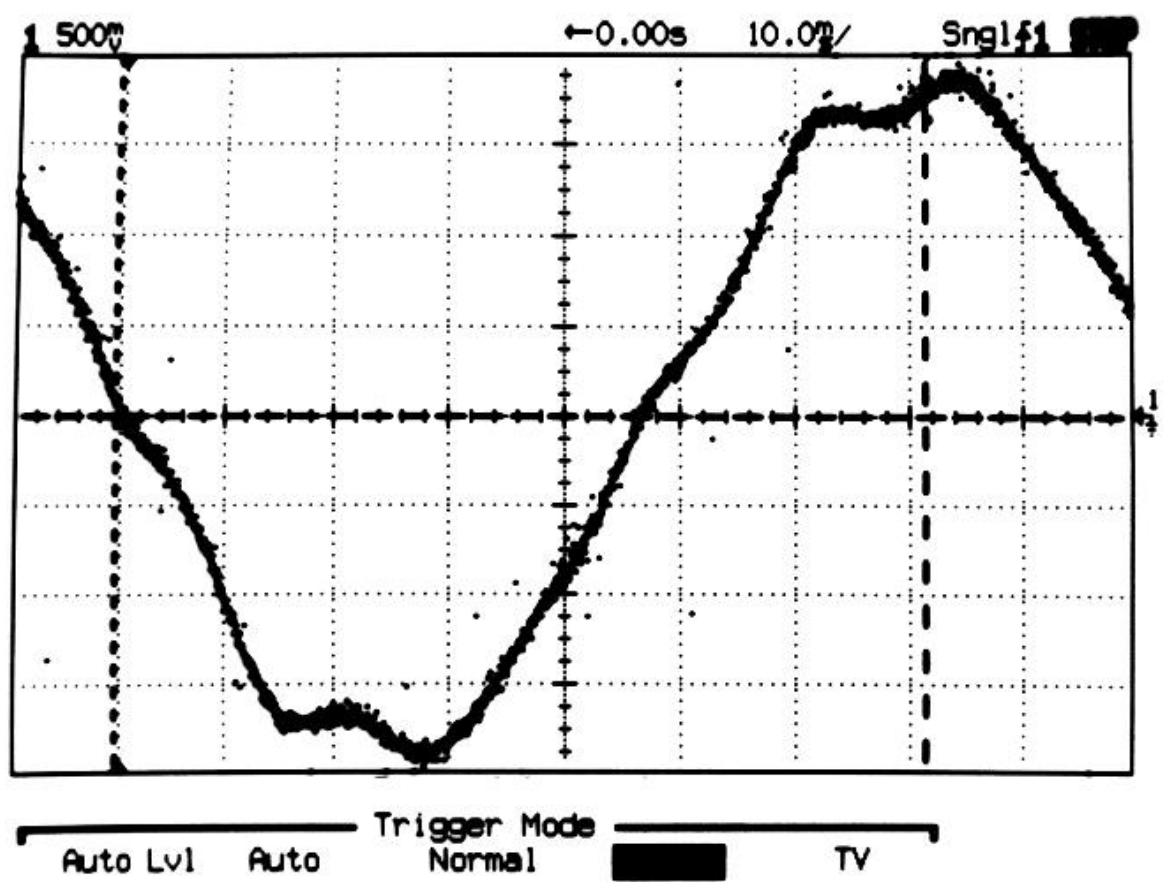

(b)

Figura 5.5: Simulação da corrente de fase e do torque eletromagnético (gráfico - a) e medida da corrente de fase (gráfico - b) na MSIP com controle vetorial em malha aberta de corrente (corrente: $1,06 \mathrm{~A} / \mathrm{div}, \omega_{m}=22,5 \mathrm{rad} / \mathrm{s}$ ). 


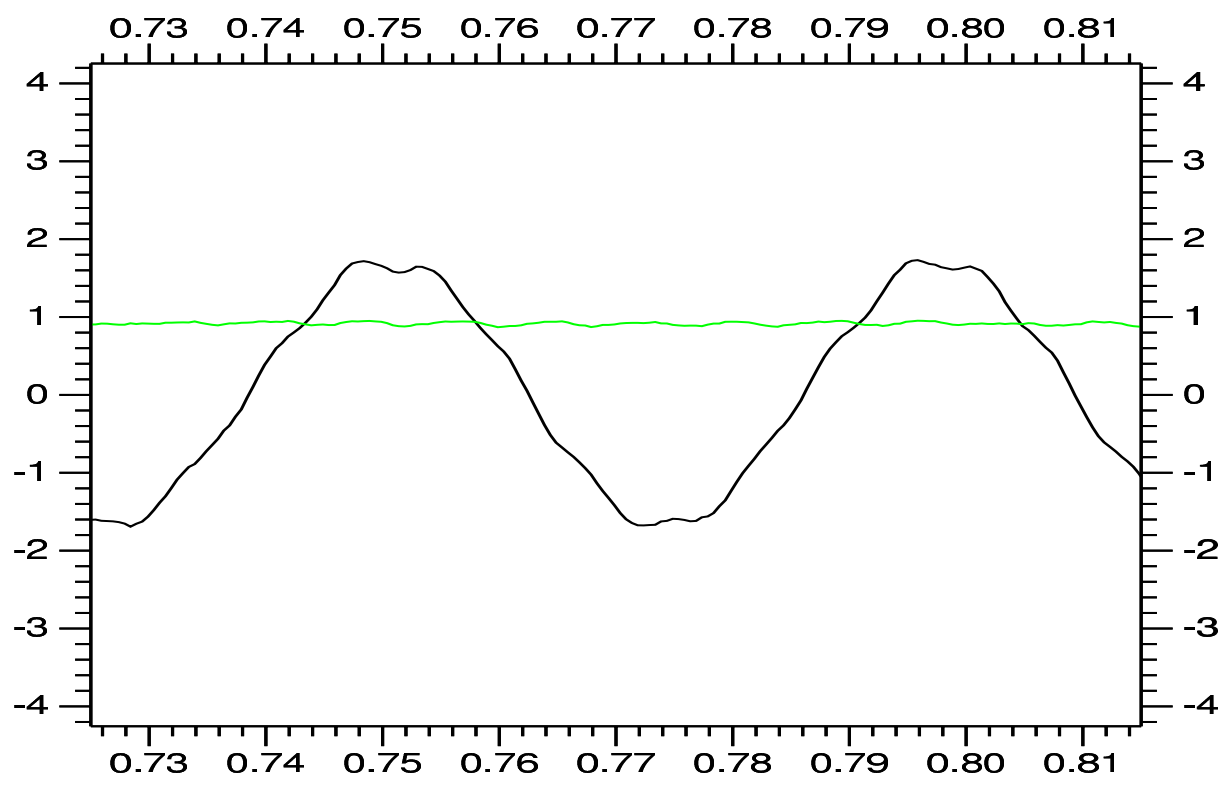

(a)

Tel (N.m)

(b)

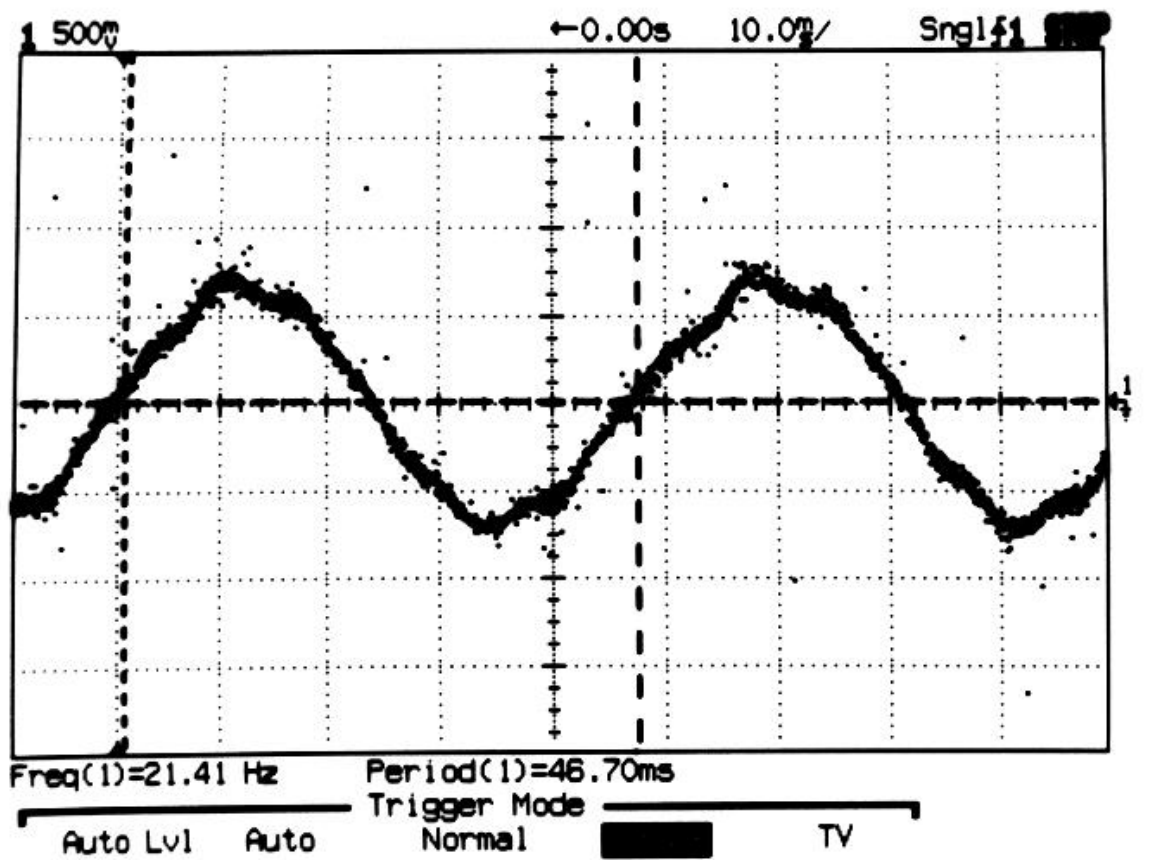

Figura 5.6: Simulação da corrente de fase e do torque eletromagnético (gráfico - a) e medida da corrente de fase (gráfico - b) na MSIP com controle vetorial em malha aberta de corrente (corrente: $1,06 \mathrm{~A} / \mathrm{div}, \omega_{m}=44,8 \mathrm{rad} / \mathrm{s}$ ). 


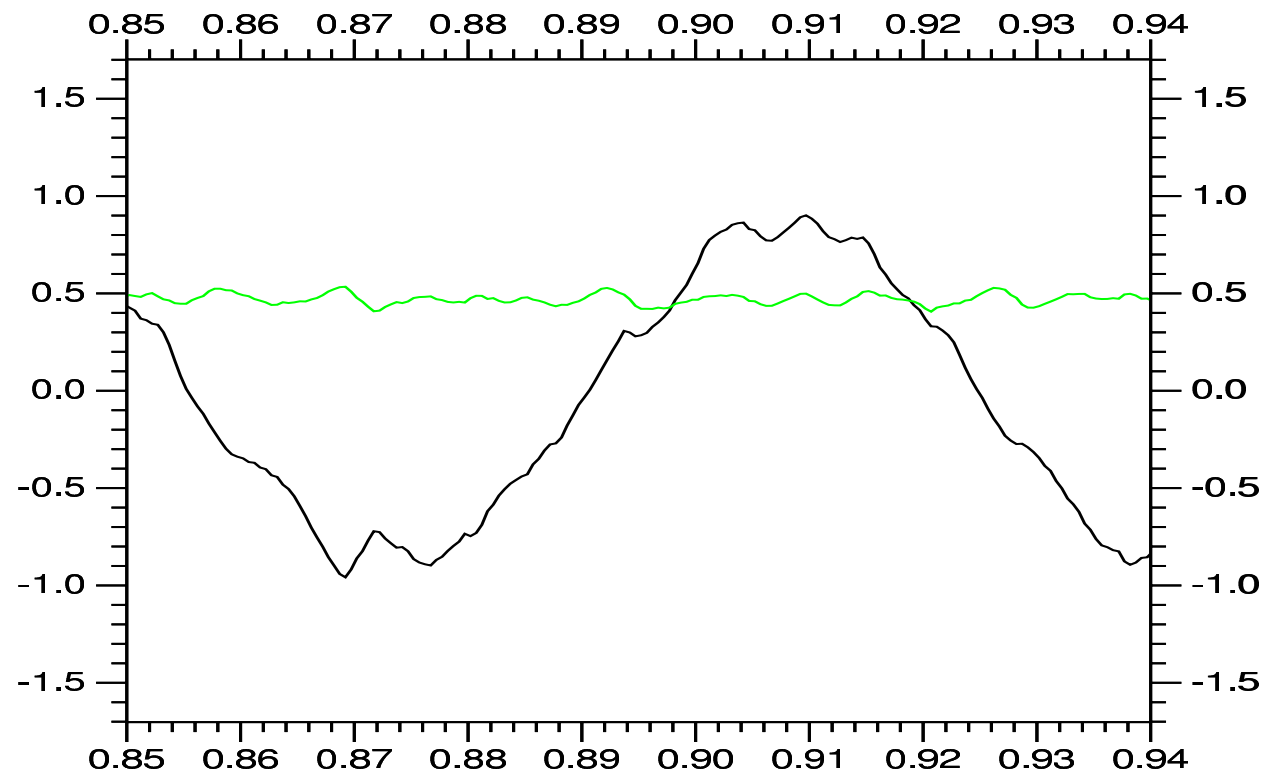

(a) ia $(\mathrm{A})$ Tel (N.m)

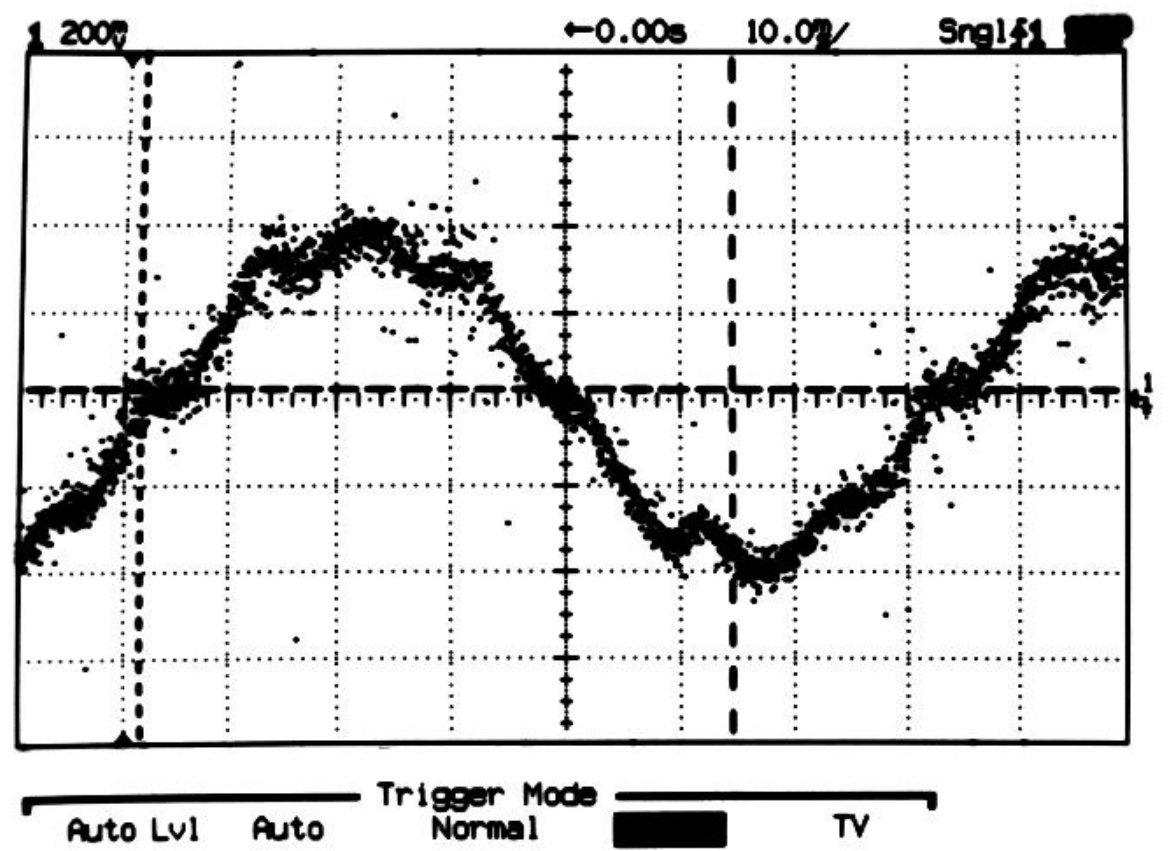

(b)

Figura 5.7: Simulação da corrente de fase e do torque eletromagnético (gráfico - a) e medida da corrente de fase (gráfico - b) na MSIP com controle vetorial em malha aberta de corrente (corrente: $0,426 \mathrm{~A} / \mathrm{div}, \omega_{m}=30,4 \mathrm{rad} / \mathrm{s}$ ). 
Esses gráficos mostram o efeito da normalização (ou discretização) e também mostram melhor ainda quão próximo o modelo da máquina está do modelo real. Nota-se que, na figura 5.7, o semi-ciclo negativo e o semi-ciclo positivo da corrente de fase da máquina são muito próximos aos da simulação.

Conforme citado anteriormente, a apresentação das figuras mostrando a forma de onda da corrente de estator obtida por simulação e pela operação da máquina, real em condições semelhantes, tem como objetivo mostrar a validade do modelo vetorial proposto.

\subsection{Medida Indireta de Torque com a Aplicação de Con- trole Vetorial em Malha Aberta de Corrente e com Acionamento no Modo "Seis-Pulsos"}

O torque eletromagnético da máquina alimentada por conversor com controle vetorial em malha aberta de corrente foi comparado com o torque da mesma alimentada por conversor no modo "seis-pulsos". Utilizou-se o controle vetorial em malha aberta de corrente, porque existem outros métodos, vetoriais ou não, que também apresentam bons resultados em malha fechada de corrente (Berendsen et al., 1993)(Grenier \& Louis, 1995)(Aghili et al., 2000). Também porque a comparação não seria válida, uma vez que o conversor no modo "seis-pulsos" está em malha aberta de corrente.

Ambas as topologias de conversores utilizam somente a informação da posição rotórica para a determinação das tensões a serem aplicadas ao estator da máquina. A diferença entre eles é que, com a utilização do modelo vetorial, necessita-se de uma maior resolução na medida do ângulo do rotor. Enquanto que no modo "seis-pulsos", necessita-se de um intervalo de posição do rotor de $60^{\circ}$ elétricos, no modo vetorial, quanto menor o intervalo de medidas, melhor o resultado do controle. Na prática, foi utilizado um encoder de 1000 pulsos por volta, o que resulta em 333,33 pulsos por volta elétrica $\left(z_{p}=3\right)$.

Para o levantamento dos resultados, a máquina foi acoplada ao eixo de uma roldana por uma barra de torção de alumínio. Na roldana, utilizou-se uma corda elástica atada a um peso de $4,5 \mathrm{~kg}$. A massa foi escolhida de tal forma que o torque aplicado pelo 


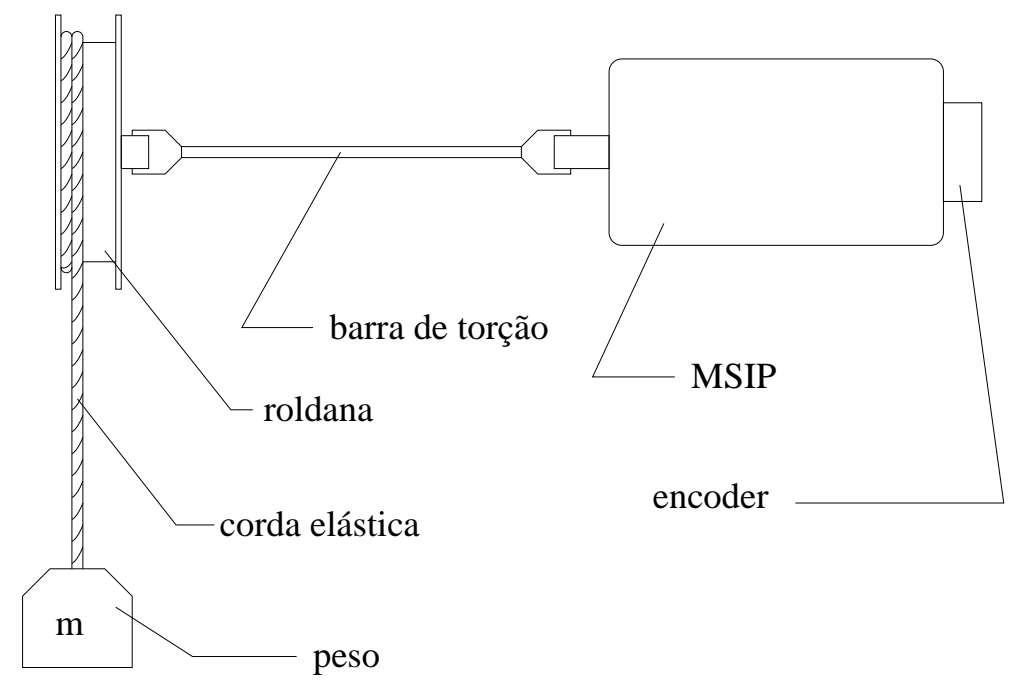

Figura 5.8: Sistema utilizado para medida da posição do rotor em função do tipo de controle utilizado.

conjunto roldana/corda/peso ao eixo do motor foi de $2,8 \mathrm{~N} \cdot \mathrm{m}$ (figura 5.8). A barra de alumínio possui $8 \mathrm{~mm}$ de diâmetro e $25 \mathrm{~cm}$ de comprimento, apresentando uma torção máxima de $10^{\circ} \mathrm{com} 10 \mathrm{~N} \cdot \mathrm{m}$ torque máximo aplicado ${ }^{1}$, ou $1^{\circ} /(\mathrm{N} \cdot \mathrm{m})$.

A barra de torção foi utilizada para que a inércia da roldana não atenuasse muito o efeito das ondulações do torque eletromagnético, uma vez que a leitura dessas é realizada indiretamente através da posição do rotor por um encoder acoplado ao eixo da máquina. Da mesma maneira, utilizou-se uma corda elástica para que a inércia da massa tivesse um efeito menor durante os transitórios de torque eletromagnético.

A máquina foi alimentada pelo conversor elétrico no modo "seis-pulsos" e com controle vetorial em malha aberta de corrente. Os resultados são apresentados nas figuras 5.9 e 5.10 .

Os gráficos das figuras mostram a posição rotórica lida pelo encoder acoplado ao eixo da MSIP em função do tempo. Em cada figura, são apresentados três gráficos (a, b e c) correspondentes a um determinado intervalo de tempo. Os gráficos 5.9(a) e 5.10(a) mostram a massa inicialmente em repouso, que em seguida, é erguida com uma velocidade aproximadamente constante. Nos gráficos 5.9(b) e 5.10(b) mostram um trecho de aproximadamente $2,5 \mathrm{~s}$ e $2 \mathrm{~s}$, respectivamente, durante o levantamento da massa. O gráfico 5.9(c) mostra o detalhe da posição rotórica em uma região de ondulação de

\footnotetext{
${ }^{1}$ Região elástica do material.
} 


\section{SSPWM}

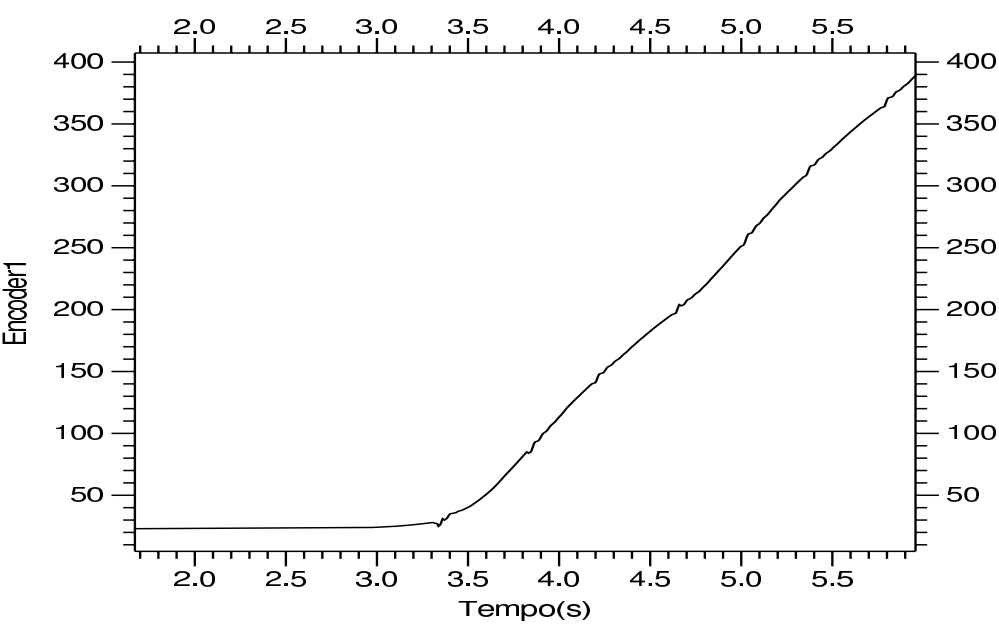

(a)

SSPWM

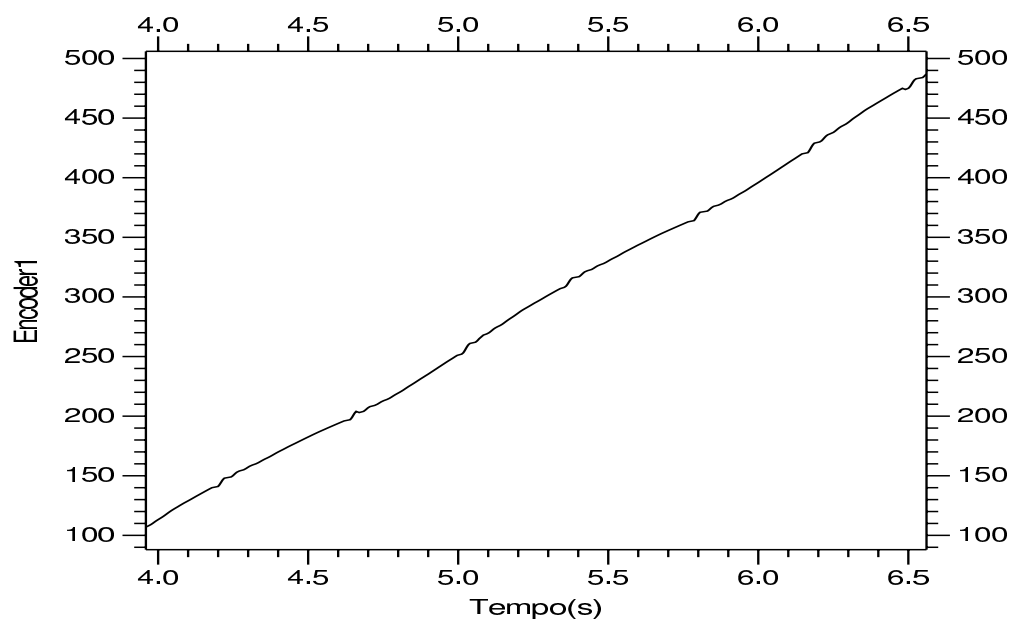

SSPWM

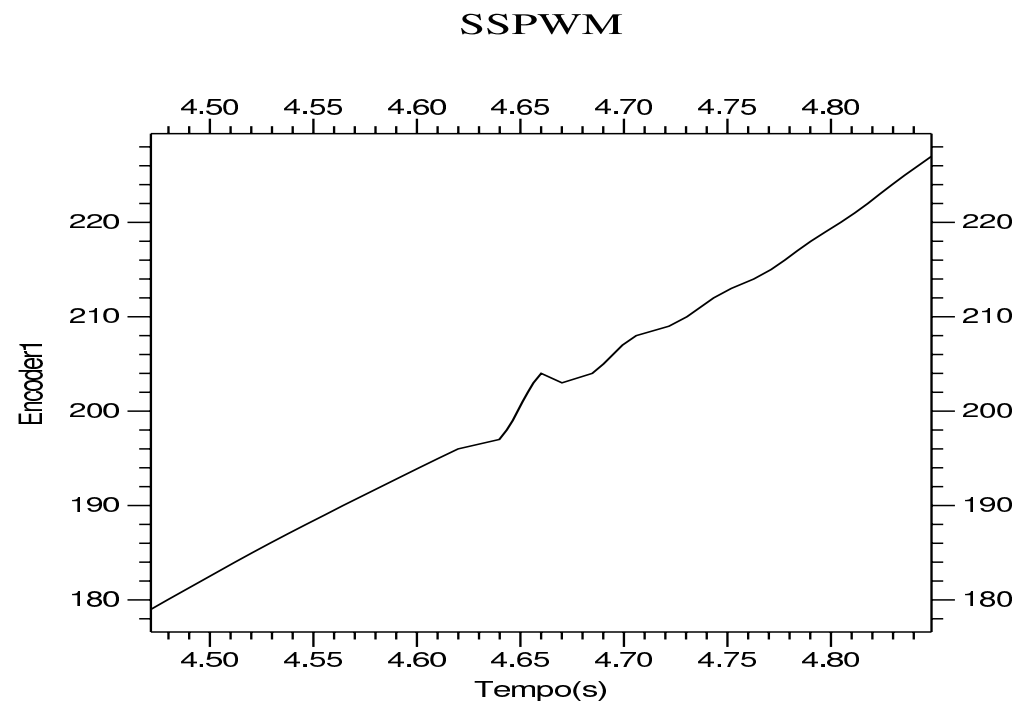

(c)

Figura 5.9: Posição rotórica da máquina ao levantar carga através de roldana, com conversor no modo "seis-pulsos". As subfiguras (b) e (c) correspondem a ampliações da subfigura (a). 


\section{OLVPWM}

(a)

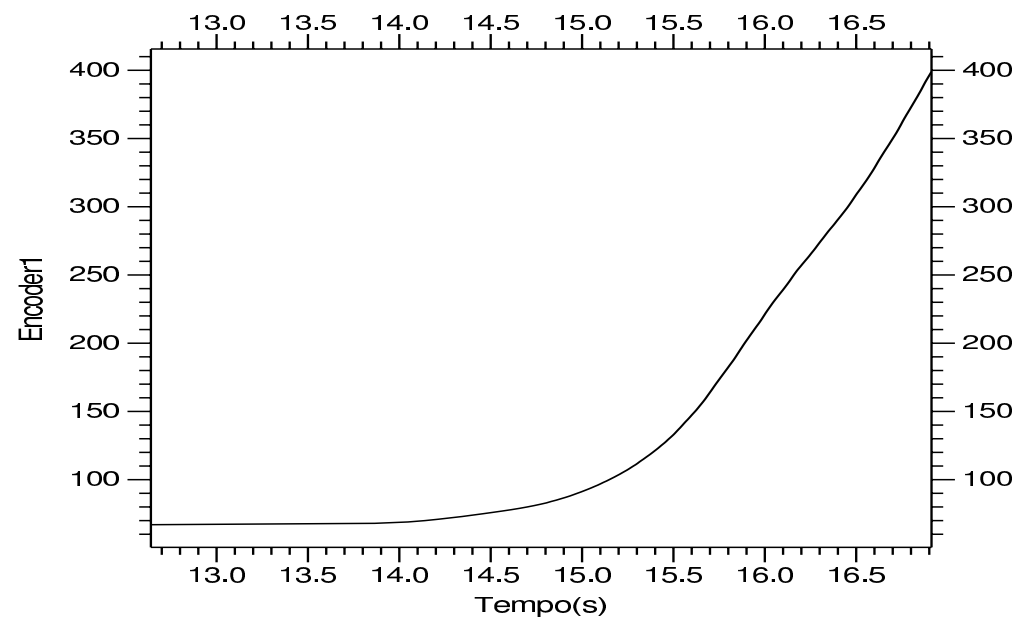

OLVPWM

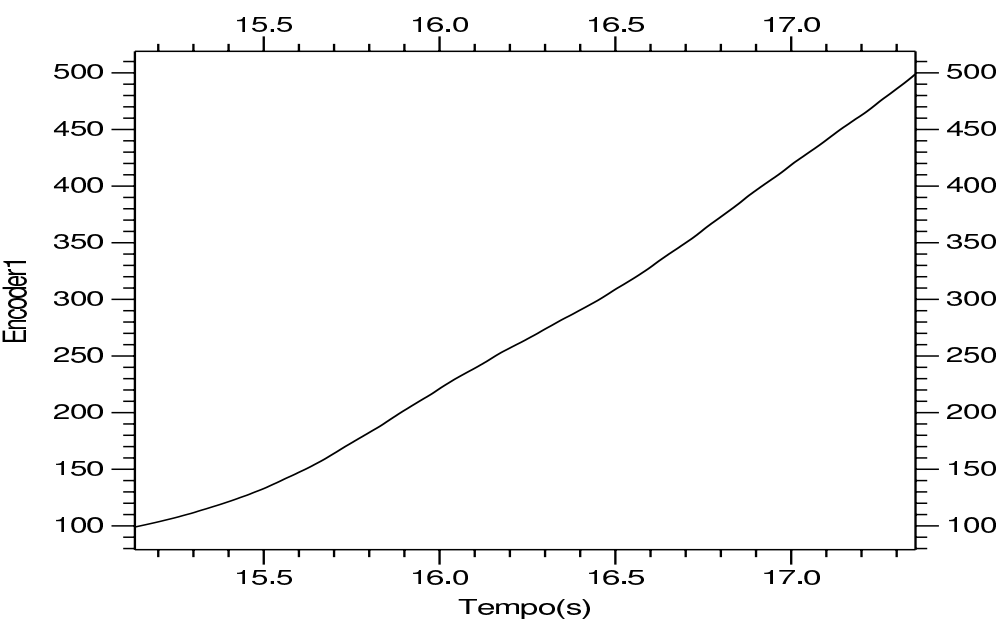

(b)

\section{OLVPWM}

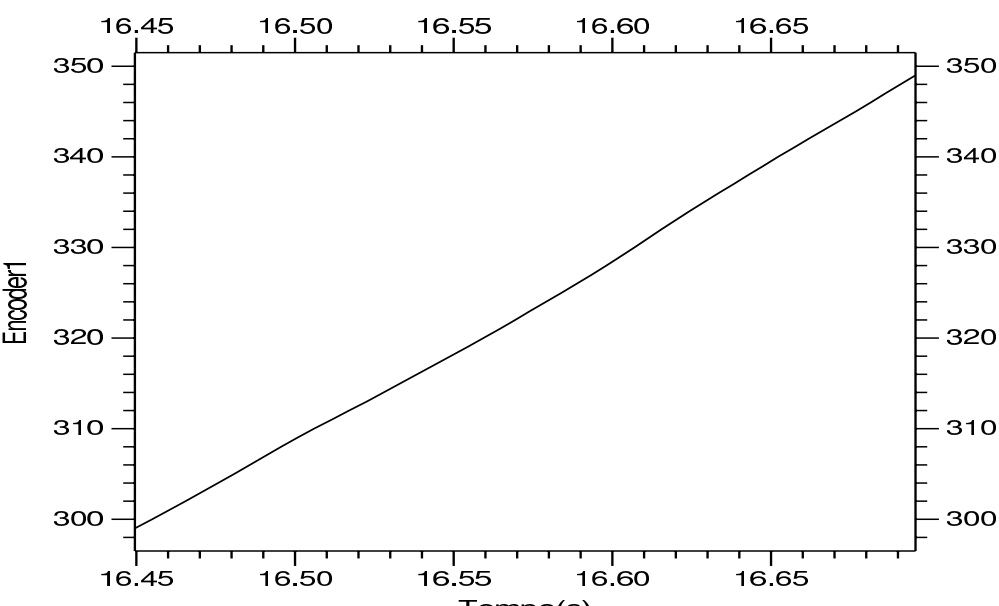

(c)

Tempo(s)

Figura 5.10: Posição rotórica da máquina ao levantar carga através de roldana, com conversor utilizando controle vetorial em malha aberta de corrente. As subfiguras (b) e (c) correspondem a ampliações da subfigura (a). 
torque eletromagnético, com o conversor no modo "seis-pulsos". O gráfico 5.10(c) mostra detalhe da posição rotórica na mesma região, mas com a máquina acionada com controle vetorial.

Nos dois experimentos, a malha de controle de velocidade não é fechada: aplica-se um torque de referência fixo ao sistema de controle, e essa referência não é alterada durante o levantamento da carga. Portanto, os gráficos podem não mostrar uma inclinação constante, devido à variações da carga como o enrolamento da corda na roldana, excentricidades da roldana, etc. Procurou-se aplicar um torque de referência que neutraliza-se a força peso da massa $(2,8 \mathrm{~N} \cdot \mathrm{m})$. Nos dois casos, a massa encontrava-se inicialmente suspensa, em equilíbrio com o torque da máquina, para depois ser erguida. É importante ressaltar que a malha de velocidade não foi fechada para que não influenciasse nos resultados, uma vez que esses foram medidos através da posição do rotor. 


\section{Parte III}

\section{Conclusões}




\section{Capítulo 6}

\section{Conclusões}

O trabalho apresenta um método para a redução de ondulações no torque eletromagnético de máquinas síncronas com ímã permanente na superfície do rotor e fluxo de entreferro não senoidal. Dentre as várias técnicas empregadas para a redução dessas ondulações, o método proposto pode ser classificado como controle de forma de onda de corrente programada, conforme visto no capítulo 2.

Harmônicos indesejáveis na forma de onda da distribuição da densidade de fluxo magnético no entreferro da máquina constituem-se no fator mais significativo para a produção de ondulações no torque eletromagnético nesse tipo de máquina. Esses harmônicos indesejáveis influem na componente de torque denominada de "torque mútuo", que é a principal componente de torque eletromagnético nesse tipo de máquina.

O método apresentado consiste em um modelo vetorial onde torna-se possível a isolação da componente da corrente de estator responsável pela produção do torque mútuo. Dessa forma, com o controle adequado dessa componente de corrente (através de uma corrente de referência baseada no torque desejado, ou torque de referência), consegue-se a forma de onda de torque eletromagnético desejada, livre de ondulações.

Através dos resultados obtidos para a corrente de estator, capítulo 5 (figuras 5.1 a 5.7), dentro da região normal de operação dessa (fora da região de saturação magnética por correntes excessivas), conclui-se que o modelo é totalmente válido, pois as correntes reais medidas das fases do estator estão muito próximas às obtidas por simulação.

Pelos resultados obtidos através da leitura da posição rotórica com o conversor no modo "seis-pulsos" e com o controle vetorial proposto (figuras 5.9 e 5.10), pode-se 
observar que a utilização do controle vetorial eliminou as ondulações de torque eletromagnético refletidas na posição do rotor. Esse fato torna viável a utilização de MSIPs não senoidais em aplicações onde somente MSIPs senoidais poderiam ser utilizadas. 
Parte IV

Apêndices 


\section{Apêndice A}

\section{Técnicas de Projeto de MSIPs}

para Redução das Ondulações de

\section{Torque Eletromagnético}

Nas técnicas de projeto de MSIPs, são discutidos tanto o projeto do rotor da máquina quanto o projeto do estator. Tanto no projeto do estator quanto no projeto do rotor, para cada tipo de MSIP (senoidal ou não senoidal), existem técnicas diferentes.

Como técnicas de projeto de estator para máquinas senoidais, pode-se destacar o encurtamento de passo dos enrolamentos do estator, a distribuição fracional dos enrolamentos e a inclinação das ranhuras do estator. Para máquinas trapezoidais, é comum utilizar-se de enrolamentos concentrados. Em ambos os tipos de MSIPs, o aumento do número de fases diminui as ondulações no torque, diminuindo sua amplitude e aumentando sua freqüência (Chan et al., 1994) (Chan et al., 1996).

Máquinas com o chamado estator liso não apresentam torque de borda (torque devido a interação das ranhuras do estator com os ímãs do rotor), pois não possuem dentes no estator (Miller, 1993).

No projeto magnético do estator, pode-se empregar pequenas ranhuras vazias (sem enrolamento) para que aumente-se a ordem dos harmônicos do torque de borda, mas diminua-se sua amplitude. Da mesma forma, a utilização de dentes vazios também produz o mesmo efeito. Essas técnicas são empregadas em máquinas com baixo número 
de ranhuras por pólo por fase.

A abertura das ranhuras pode ser diminuída para que com isto diminua-se a amplitude do torque de borda. Essa diminuição apresenta a desvantagem de introduzir harmônicos indesejáveis na forma de onda da FEM, levando a produção de outros harmônicos no torque eletromagnético (Sebastian \& Gangla, 1996).

As técnicas de projeto de rotor também vão ser distintas dependendo do tipo de MSIP, senoidal ou não senoidal. São citadas tanto diferentes direções de magnetização dos ímãs quanto diferentes distribuições de magnetização desses.

O comprimento do arco do ímã no rotor e seu posicionamento também podem ser determinados de tal forma que produzam harmônicos com amplitudes mais baixas no torque eletromagnético. Ímãs colocados de forma discretizada no rotor também podem reduzir esses harmônicos, dependendo de sua distribuição. Os ímãs também podem ser utilizados em pares de arco, sendo que o posicionamento entre eles pode ser determinado para que se produzam menores ondulações no torque.

Pode-se também inclinar os ímãs no rotor a fim de se diminuir componentes harmônicos de alta ordem no torque eletromagnético. 


\section{Apêndice B}

\section{Modelagem Convencional por}

\section{Fase}

Nesse apêndice, a dedução das equações 3.1 e 3.2 é apresentada.

A figura B.1 representa uma MSIP de forma simplificada (reprodução da figura 3.1). Com relação a essa figura, escreve-se a equação de fluxo total:

$$
\left[\begin{array}{c}
\Phi_{t s a} \\
\Phi_{t s b} \\
\Phi_{t s c}
\end{array}\right]=\left[\begin{array}{c}
\Phi_{s a} \\
\Phi_{s b} \\
\Phi_{s c}
\end{array}\right]+\left[\begin{array}{c}
\Phi_{r a} \\
\Phi_{r b} \\
\Phi_{r c}
\end{array}\right]
$$

Onde:

$\Phi_{t s a}, \Phi_{t s b}$ e $\Phi_{t s c}$ : fluxo magnético total concatenado pelas fases a, b e c do estator;

$\Phi_{s a}, \Phi_{s b}$ e $\Phi_{s c}$ : fluxo magnético concatenado pelas fases a, b e c do estator, produzido somente pelos enrolamentos do estator;

$\Phi_{r a}, \Phi_{r b}$ e $\Phi_{r c}$ fluxo magnético concatenado pelas fases a, b e c do estator, produzido somente pelos ímãs do rotor.

Dessa forma, as tensões de estator são dadas por: 


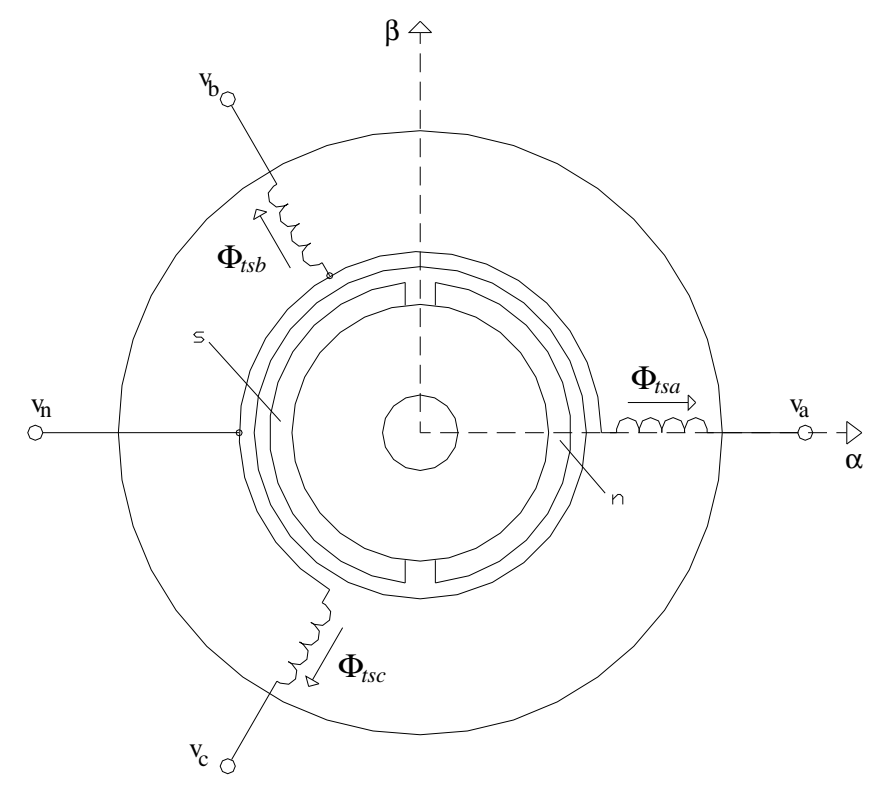

Figura B.1: Esquema físico simplificado do servo-motor (motor com 2 pólos).

$$
\left[\begin{array}{c}
v_{a n} \\
v_{b n} \\
v_{c n}
\end{array}\right]=R_{s}\left[\begin{array}{c}
i_{a} \\
i_{b} \\
i_{c}
\end{array}\right]+\frac{d}{d t}\left[\begin{array}{c}
\Phi_{t s a} \\
\Phi_{t s b} \\
\Phi_{t s c}
\end{array}\right]=R_{s}\left[\begin{array}{c}
i_{a} \\
i_{b} \\
i_{c}
\end{array}\right]+\frac{d}{d t}\left(\left[\begin{array}{c}
\Phi_{s a} \\
\Phi_{s b} \\
\Phi_{s c}
\end{array}\right]+\left[\begin{array}{c}
\Phi_{r a} \\
\Phi_{r b} \\
\Phi_{r c}
\end{array}\right]\right)
$$

E como os fluxos magnéticos produzidos pelo estator podem ser descritos pela equação B.3, a equação elétrica da máquina é escrita pela equação B.4.

$$
\begin{aligned}
& {\left[\begin{array}{c}
\Phi_{s a} \\
\Phi_{s b} \\
\Phi_{s c}
\end{array}\right]=L\left[\begin{array}{c}
i_{a} \\
i_{b} \\
i_{c}
\end{array}\right]=\left[\begin{array}{ccc}
L_{s} & M_{s} & M_{s} \\
M_{s} & L_{s} & M_{s} \\
M_{s} & M_{s} & L_{s}
\end{array}\right]\left[\begin{array}{c}
i_{a} \\
i_{b} \\
i_{c}
\end{array}\right]} \\
& {\left[\begin{array}{c}
v_{a n} \\
v_{b n} \\
v_{c n}
\end{array}\right]=R_{s}\left[\begin{array}{c}
i_{a} \\
i_{b} \\
i_{c}
\end{array}\right]+L \frac{d}{d t}\left[\begin{array}{c}
i_{a} \\
i_{b} \\
i_{c}
\end{array}\right]+\frac{d}{d t}\left[\begin{array}{c}
\Phi_{r a} \\
\Phi_{r b} \\
\Phi_{r c}
\end{array}\right]}
\end{aligned}
$$

Quanto aos fluxos magnéticos produzidos pelo rotor e concatenados pelo estator, pode-se escrever: 


$$
\frac{d}{d t}\left[\begin{array}{c}
\Phi_{r a} \\
\Phi_{r b} \\
\Phi_{r c}
\end{array}\right]=\left[\begin{array}{c}
e_{a} \\
e_{b} \\
e_{c}
\end{array}\right]
$$

Da mesma forma, pode-se desenvolver a equação B.5:

$$
\frac{d}{d t}\left[\begin{array}{c}
\Phi_{r a} \\
\Phi_{r b} \\
\Phi_{r c}
\end{array}\right]=\omega_{r} \frac{d}{d \theta_{r}}\left[\begin{array}{c}
\Phi_{r a} \\
\Phi_{r b} \\
\Phi_{r c}
\end{array}\right]
$$

Da equação B.6, os termos $\Phi_{r a}^{\prime}, \Phi_{r b}^{\prime}$ e $\Phi_{r c}^{\prime}$ dependem do ângulo do rotor $\left(\theta_{r}\right)$ e não variam com a velocidade, desde que a máquina esteja operando dentro da região linear, fora da região de saturação. Pode-se normalizar esses termos com a equação B.7, de tal forma que $\Phi_{m}$ seja escolhido fazendo com que as formas de onda $F_{r a}, F_{r b}$ e $F_{r c}$ possuam valor máximo igual a 1 e mínimo igual a -1 (ou valores máximos e mínimos próximos a esses valores, no caso de máquinas que apresentem assimetrias).

$$
\frac{d}{d \theta_{r}}\left[\begin{array}{c}
\Phi_{r a} \\
\Phi_{r b} \\
\Phi_{r c}
\end{array}\right]=\Phi_{m}\left[\begin{array}{c}
F_{r a} \\
F_{r b} \\
F_{r c}
\end{array}\right]
$$

E, portanto:

$$
\left[\begin{array}{c}
e_{a} \\
e_{b} \\
e_{c}
\end{array}\right]=\omega_{r} \Phi_{m}\left[\begin{array}{c}
F_{r a} \\
F_{r b} \\
F_{r c}
\end{array}\right]
$$

Desenvolvendo-se a equação B.4, tem-se:

$$
\left[\begin{array}{c}
v_{a} \\
v_{b} \\
v_{c}
\end{array}\right]=R_{s}\left[\begin{array}{c}
i_{a} \\
i_{b} \\
i_{c}
\end{array}\right]+L \frac{d}{d t}\left[\begin{array}{c}
i_{a} \\
i_{b} \\
i_{c}
\end{array}\right]+\left[\begin{array}{c}
e_{a} \\
e_{b} \\
e_{c}
\end{array}\right]+\left[\begin{array}{c}
v_{n} \\
v_{n} \\
v_{n}
\end{array}\right]
$$

Ou: 


$$
\left[\begin{array}{c}
v_{a} \\
v_{b} \\
v_{c}
\end{array}\right]=R_{s}\left[\begin{array}{c}
i_{a} \\
i_{b} \\
i_{c}
\end{array}\right]+L \frac{d}{d t}\left[\begin{array}{c}
i_{a} \\
i_{b} \\
i_{c}
\end{array}\right]+\omega_{r} \Phi_{m}\left[\begin{array}{c}
F_{r a} \\
F_{r b} \\
F_{r c}
\end{array}\right]+\left[\begin{array}{c}
v_{n} \\
v_{n} \\
v_{n}
\end{array}\right]
$$

Analisando-se a equação B.4 e B.5, conclui-se que a potência transferida ao rotor da máquina é dada pela equação B.11.

$$
P_{\text {rotor }}=\left[\begin{array}{c}
i_{a} \\
i_{b} \\
i_{c}
\end{array}\right]^{t}\left[\begin{array}{l}
e_{a} \\
e_{b} \\
e_{c}
\end{array}\right]
$$

A potência mecânica desenvolvida pela máquina no eixo do rotor é dada pela equação B.12.

$$
P_{\text {rotor }}=T_{e l} \omega_{m}
$$

Onde:

$\omega_{m}$ : velocidade mecânica do rotor em rad/s (equação B.13).

$$
\omega_{m}=\frac{\omega_{r}}{z_{p}}
$$

Dessa forma, pode-se escrever:

$$
T_{e l} \omega_{m}=\left[\begin{array}{c}
i_{a} \\
i_{b} \\
i_{c}
\end{array}\right]^{t}\left[\begin{array}{c}
e_{a} \\
e_{b} \\
e_{c}
\end{array}\right]=\left[\begin{array}{c}
i_{a} \\
i_{b} \\
i_{c}
\end{array}\right]^{t}\left(\omega_{r} \Phi_{m}\left[\begin{array}{c}
F_{r a} \\
F_{r b} \\
F_{r c}
\end{array}\right]\right)
$$

Portanto, a equação final para o torque eletromagnético é a equação B.15.

$$
T_{e l}=z_{p} \omega_{r} \Phi_{m}\left[\begin{array}{c}
i_{a} \\
i_{b} \\
i_{c}
\end{array}\right]^{t}\left[\begin{array}{c}
F_{r a} \\
F_{r b} \\
F_{r c}
\end{array}\right]
$$




\section{Apêndice $\mathrm{C}$}

\section{Transformações de Coordenadas e Modelos Vetoriais da Máquina}

\section{C.1 Transformações de Coordenadas}

Para a modelagem vetorial da máquina, serão consideradas algumas transformações de coordenadas. A primeira transformação de coordenadas considerada é a Transformação $\alpha \beta 0^{1}$, expressa na equações C.1 e C.2, em suas formas complexas.

$$
\begin{gathered}
x_{\alpha \beta}=\sqrt{\frac{2}{3}}\left[\begin{array}{lll}
1 & e^{j \frac{2 \pi}{3}} & e^{-j \frac{2 \pi}{3}}
\end{array}\right]\left[\begin{array}{l}
x_{a} \\
x_{b} \\
x_{c}
\end{array}\right] \\
x_{0}=\sqrt{\frac{2}{3}}\left[\begin{array}{lll}
\frac{\sqrt{2}}{2} & \frac{\sqrt{2}}{2} & \frac{\sqrt{2}}{2}
\end{array}\right]\left[\begin{array}{c}
x_{a} \\
x_{b} \\
x_{c}
\end{array}\right]
\end{gathered}
$$

Onde:

$x_{\alpha \beta}$ : grandezas representadas nos eixos $\alpha \beta$, na forma complexa;

$x_{0}$ : componente zero;

$x_{a}, x_{b}$ e $x_{c}$ : grandezas representadas nas fases a, b e c, respectivamente.

\footnotetext{
${ }^{1}$ Também denominada de transformação Concordia por alguns autores.
} 
Ou então, na forma matricial:

$$
\left[\begin{array}{l}
x_{a} \\
x_{b} \\
x_{c}
\end{array}\right]=T\left[\begin{array}{l}
x_{\alpha} \\
x_{\beta} \\
x_{0}
\end{array}\right] \Rightarrow T=\sqrt{\frac{2}{3}}\left[\begin{array}{ccc}
1 & 0 & \frac{\sqrt{2}}{2} \\
-\frac{1}{2} & \frac{\sqrt{3}}{2} & \frac{\sqrt{2}}{2} \\
-\frac{1}{2} & -\frac{\sqrt{3}}{2} & \frac{\sqrt{2}}{2}
\end{array}\right]
$$

A outra transformação considerada é a Transformação $d q$, com ângulo dos eixos $d q$ $\left(\theta_{d q}\right)$ igual ao ângulo do rotor $\left(\theta_{r}\right)$, expressa na equação C.4.

$$
x_{\alpha \beta}=e^{j \theta_{r}} x_{d q}
$$

Onde:

$x_{d q}$ : grandezas representadas nos eixos $d q$, na forma complexa;

Essa transformação é empregada em MSIP com fluxo de entreferro senoidal, pois o ângulo do rotor é igual ao ângulo do campo da máquina.

As transformações de coordenadas acima são aplicadas nas equações de fase da máquina (equações 3.1 e 3.2) a fim de se obter as equações vetoriais da mesma.

Com relação à transformação $d q$, pode-se utilizar algumas variações da mesma. Pode-se considerar o ângulo dos eixos $d q$ igual ao ângulo do rotor da máquina (como é o caso da transformação da equação C.4), ou então, pode-se considerar o ângulo dos eixos $d q$ qualquer, como apresentado em (Grenier \& Louis, 1995). Nesse caso, a transformação $d q$ é indicada como na equação C.5, onde o ângulo da transformação é indicado simplesmente por $\theta_{d q}$.

$$
x_{\alpha \beta}=e^{j \theta_{d q}} x_{d q}
$$

\section{C.2 Aplicação das Transformações de Coordenadas no Mo- delo da Máquina}

A transformação $\alpha \beta 0$ é aplicada às equações elétricas e de torque eletromagnético (equações 3.1 e 3.2). Nas equações resultantes, são aplicadas ou a transformação dada 
pela equação C.4 (para o caso senoidal) ou a transformação dada pela equação C.5 (para o caso não senoidal).

\section{C.2.1 Aplicação da Transformação $\alpha \beta 0$}

Ao se aplicar a transformação $\alpha \beta 0$ nas equações elétricas e de torque eletromagnético (equação 3.1 e 3.2) obtém-se as equações C.6, C.7 e C.8

$$
\begin{gathered}
v_{\alpha \beta}=R_{s} i_{\alpha \beta}+\left(L_{s}-M_{s}\right) \frac{d}{d t} i_{\alpha \beta}+\omega_{r} \Phi_{m} F_{r \alpha \beta} \\
v_{0}=R_{s} i_{0}+\left(L_{s}+2 M_{s}\right) \frac{d}{d t} i_{0}+\omega_{r} \Phi_{m} F_{r 0}+\sqrt{3} v_{n} \\
T_{e l}=z_{p} \Phi_{m}\left(i_{\alpha} F_{r \alpha}+i_{\beta} F_{r \beta}+i_{0} F_{r 0}\right)
\end{gathered}
$$

Onde:

$v_{\alpha \beta}$ : tensão aplicada ao estator representada nos eixos $\alpha \beta$ (forma complexa:

$$
\left.v_{\alpha}+j v_{\beta}\right)
$$

$v_{0}$ : componente zero da tensão aplicada ao estator;

$i_{\alpha \beta}$ : corrente de estator representada nos eixos $\alpha \beta$ (forma complexa: $i_{\alpha}+$ $\left.j i_{\beta}\right)$;

$i_{0}$ : componente zero da corrente de estator;

$F_{r \alpha \beta}$ : forma de onda de FEM normalizada, representada nos eixos $\alpha \beta$ (forma complexa: $F_{r \alpha}+j F_{r \beta}$ );

$F_{r 0}$ : componente zero da forma de onda de FEM normalizada.

Normalmente as máquinas não possuem ligação do terminal central, portanto a corrente de componente zero é nula $\left(i_{0}=0\right)$. Desse modo, a equação do torque eletromagnético (equação C.8) passa a ser dada pela equação C.9.

$$
T_{e l}=z_{p} \Phi_{m}\left(i_{\alpha} F_{r \alpha}+i_{\beta} F_{r \beta}\right)
$$




\section{C.2.2 Aplicação da Transformação $d q$ com Ângulo dos Eixos $d q$ Igual ao Ângulo do Rotor da Máquina}

Essa transformação é utilizada em MSIPs com forma de onda de FEM senoidal.

Aplicando-se a transformação dada pela equação C.4 nas equações obtidas com a aplicação da transformação $\alpha \beta$ (equações C.6 e C.9), obtém-se a equação C.10, na forma complexa, e a equação C.11, para o torque eletromagnético.

$$
\begin{gathered}
v_{d q}=R_{s} i_{d q}+\left(L_{s}-M_{s}\right) \frac{d}{d t} i_{d q}+j\left(L_{s}-M_{s}\right) \omega_{r} i_{d q}+\omega_{r} \Phi_{m} F_{r d q} \\
T_{e l}=z_{p} \Phi_{m}\left(i_{d} F_{r d}+i_{q} F_{r q}\right)
\end{gathered}
$$

Onde:

$v_{d q}$ : tensão aplicada ao estator representada nos eixos $d q$ (forma complexa:

$$
\left.v_{d}+j v_{q}\right)
$$

$i_{d q}$ : corrente de estator representada nos eixos $d q$ (forma complexa: $i_{d}+j i_{q}$ );

$F_{r d q}:$ forma de onda de FEM normalizada, representada nos eixos $d q$ (forma complexa: $\left.F_{r d}+j F_{r q}\right)$;

Sendo a forma de onda do fluxo de entreferro senoidal (ou cossenoidal, ver figura 3.1), pode-se então escrever que:

$$
\begin{aligned}
& \Phi_{r a}=\Phi_{m} \cos \left(\theta_{r}\right) \\
& \Phi_{r b}=\Phi_{m} \cos \left(\theta_{r}-\frac{2 \pi}{3}\right) \\
& \Phi_{r c}=\Phi_{m} \cos \left(\theta_{r}+\frac{2 \pi}{3}\right)
\end{aligned}
$$

Então, para a forma de onda de FEM, tem-se: 


$$
\begin{aligned}
& F_{r a}=-\operatorname{sen}\left(\theta_{r}\right) \\
& F_{r b}=-\operatorname{sen}\left(\theta_{r}-\frac{2 \pi}{3}\right) \\
& F_{r c}=-\operatorname{sen}\left(\theta_{r}+\frac{2 \pi}{3}\right)
\end{aligned}
$$

Isso implica que:

$$
F_{r d q}=j \sqrt{\frac{3}{2}}
$$

Ou seja: $F_{r d}=0$ e $F_{r q}=\sqrt{\frac{3}{2}}$.

Dessa forma, as equações elétricas (na forma real) e de torque eletromagnético, para uma MSIP com forma de onda de fluxo de entreferro senoidal, são apresentadas nas equações C.15, C.16 e C.17.

$$
\begin{gathered}
v_{d}=R_{s} i_{d}+\left(L_{s}-M_{s}\right) \frac{d}{d t} i_{d}-\left(L_{s}-M_{s}\right) \omega_{r} i_{q} \\
v_{q}=R_{s} i_{q}+\left(L_{s}-M_{s}\right) \frac{d}{d t} i_{q}+\left(L_{s}-M_{s}\right) \omega_{r} i_{d}+\sqrt{\frac{3}{2}} \Phi_{m} \omega_{r} \\
T_{e l}=z_{p} \sqrt{\frac{3}{2}} \Phi_{m} i_{q}
\end{gathered}
$$

\section{C.2.3 Aplicação da Transformação $d q$ com Ângulo dos Eixos $d q$ Qual- quer}

Essa transformação é utilizada para máquinas com fluxo de entreferro não senoidal e foi proposta inicialmente em (Grenier \& Louis, 1995). A diferença da utilização dessa transformação ao invés da transformação proposta no presente trabalho é que, nesse caso, a equação do torque eletromagnético fica mais complexa, sendo uma função não linear de $\theta_{r}$ e $i_{q}$ (equação C.24).

Com a aplicação da transformação de coordenadas dada pela equação C.5 nas equações C.6 e C.9, obtém-se as equações C.18 e C.19. 


$$
\begin{gathered}
v_{d q}=R_{s} i_{d q}+\left(L_{s}-M_{s}\right) \frac{d}{d t} i_{d q}+j\left(L_{s}-M_{s}\right) \omega_{r} \frac{d \theta_{d q}}{d \theta_{r}} i_{d q}+\omega_{r} \Phi_{m} F_{r d q} \\
T_{e l}=z_{p} \Phi_{m}\left(i_{d} F_{r d}+i_{q} F_{r q}\right)
\end{gathered}
$$

A fim de se simplificar a equação de torque eletromagnético acima, impõe-se que $F_{r d}=0$. Dessa forma:

$$
F_{r q}=\sqrt{F_{r \alpha}^{2}+F_{r \beta}^{2}}
$$

Com o resultado anterior, é possível determinar a posição do ângulo dos eixos $d q$ $\left(\theta_{d q}\right)$. O valor desse ângulo é dado pela equação a seguir:

$$
\theta_{d q}=\arctan \frac{-F_{r \alpha}}{F_{r \beta}}
$$

Sendo assim, as equações elétricas da máquina (na forma real) e a equação do torque eletromagnético são escritas pelas equações C.22, C.23 e C.24.

$$
\begin{gathered}
v_{d}=R_{s} i_{d}+\left(L_{s}-M_{s}\right) \frac{d}{d t} i_{d}-\left(L_{s}-M_{s}\right) \omega_{r} \frac{d \theta_{d q}}{d \theta_{r}} i_{q} \\
v_{q}=R_{s} i_{q}+\left(L_{s}-M_{s}\right) \frac{d}{d t} i_{q}+\left(L_{s}-M_{s}\right) \omega_{r} \frac{d \theta_{d q}}{d \theta_{r}} i_{d}+\omega_{r} \Phi_{m} F_{r q} \\
T_{e l}=z_{p} i_{q} \Phi_{m} F_{r q}
\end{gathered}
$$

Nota-se que na equação do torque eletromagnético (equação C.24) existe o termo $F_{r q}$ que não é uma constante, e sim, uma função de $\theta_{r}$ (equação C.20). 


\section{Apêndice D}

\section{Dedução das equações}

\section{D.1 Dedução das equações do capítulo 3}

Dedução das equações 3.8 e 3.9 a partir das equações C.6 e C.8. A equação 3.8 é mostrada na equação D.7, e a equação 3.9, na equação D.13.

$$
v_{\alpha \beta}=R_{s} i_{\alpha \beta}+\left(L_{s}-M_{s}\right) \frac{d}{d t} i_{\alpha \beta}+\omega_{r} \Phi_{m} F_{r \alpha \beta}
$$

Se $x_{\alpha \beta}=c_{x} e^{j \theta_{r}} x_{d q_{x}}$, então:

$$
\begin{aligned}
& v_{d q_{x}} c_{x} e^{j \theta_{r}}= R_{s} i_{d q_{x}} c_{x} e^{j \theta_{r}}+\left(L_{s}-M_{s}\right) \frac{d}{d t}\left(i_{d q_{x}} c_{x} e^{j \theta_{r}}\right)+\omega_{r} \Phi_{m} F_{r d q_{x}} c_{x} e^{j \theta_{r}} \\
& v_{d q_{x}}=R_{s} i_{d q_{x}}+\frac{\left(L_{s}-M_{s}\right)}{c_{x} e^{j \theta_{r}}} \frac{d}{d t}\left(i_{d q_{x}} c_{x} e^{j \theta_{r}}\right)+\omega_{r} \Phi_{m} F_{r d q_{x}} \\
& v_{d q_{x}=} \\
& R_{s} i_{d q_{x}}+ \\
& \frac{\left(L_{s}-M_{s}\right)}{c_{x} e^{j \theta_{r}}}\left(\frac{d}{d t} i_{d q_{x}} c_{x} e^{j \theta_{r}}+i_{d q_{x}} e^{j \theta_{r}} \frac{d c_{x}}{d t}+i_{d q_{x}} c_{x} \frac{d e^{j \theta_{r}}}{d t}\right)+ \\
& \omega_{r} \Phi_{m} F_{r d q_{x}}
\end{aligned}
$$




$$
\begin{gathered}
v_{d q_{x}}=R_{s} i_{d q_{x}}+ \\
\left(L_{s}-M_{s}\right)\left(\frac{d i_{d q_{x}}}{d t}+\frac{i_{d q_{x}}}{c_{x}} \frac{d c_{x}}{d t}+j i_{d q_{x}} \omega_{r}\right)+\omega_{r} \Phi_{m} F_{r d q_{x}} \\
v_{d q_{x}}=R_{s} i_{d q_{x}}+\left(L_{s}-M_{s}\right)\left(\frac{d i_{d q_{x}}}{d t}+\frac{i_{d q_{x}}}{c_{x}} \frac{d c_{x}}{d t}+j i_{d q_{x}} \omega_{r}\right)+\omega_{r} \Phi_{m} F_{r d q_{x}} \\
v_{d q_{x}}=R_{s} i_{d q_{x}}+\left(L_{s}-M_{s}\right)\left(\frac{d i_{d q_{x}}}{d t}+i_{d q_{x}} \omega_{r}\left(\frac{1}{c_{x}} \frac{d c_{x}}{d \theta_{r}}+j\right)\right)+\omega_{r} \Phi_{m} F_{r d q_{x}} \\
T_{e l}=z_{p} \Phi_{m}\left(i_{\alpha} F_{r \alpha}+i_{\beta} F_{r \beta}+i_{0} F_{r 0}\right)
\end{gathered}
$$

Como $i_{0}=0$ :

$$
\begin{gathered}
T_{e l}=z_{p} \Phi_{m}\left(i_{\alpha} F_{r \alpha}+i_{\beta} F_{r \beta}\right)=z_{p} \Phi_{m} \operatorname{Re}\left(F_{r \alpha \beta} \cdot i_{\alpha \beta}^{*}\right) \\
T_{e l}=z_{p} \Phi_{m} \operatorname{Re}\left(c_{x} e^{j \theta_{r}} F_{r d q_{x}}\left(c_{x} e^{j \theta_{r}} i_{d q_{x}}\right)^{*}\right) \\
T_{e l}=z_{p} \Phi_{m} \operatorname{Re}\left(c_{x} e^{j \theta_{r}} F_{r d q_{x}} c_{x}^{*}\left(e^{j \theta_{r}}\right)^{*} i_{d q_{x}}^{*}\right)
\end{gathered}
$$

Como $c_{x} c_{x}^{*}=a_{x}^{2}$ e $e^{j \theta_{r}}\left(e^{j \theta_{r}}\right)^{*}=1$, então:

$$
\begin{gathered}
T_{e l}=z_{p} \Phi_{m} a_{x}^{2} \operatorname{Re}\left(F_{r d q_{x}} \cdot i_{d q_{x}}^{*}\right) \\
T_{e l}=z_{p} \Phi_{m} a_{x}^{2}\left(F_{r d_{x}} i_{d_{x}}+F_{r q_{x}} i_{q_{x}}\right)
\end{gathered}
$$

Para a equação 3.10 a partir da equação 3.8 , temos: 


$$
\begin{aligned}
& v_{d q_{x}}=R_{s} i_{d q_{x}}+\left(L_{s}-M_{s}\right)\left(\frac{d i_{d q_{x}}}{d t}+i_{d q_{x}} \omega_{r}\left(\frac{1}{a_{x} e^{j \theta_{x}}} \frac{d a_{x} e^{j \theta_{x}}}{d \theta_{r}}+j\right)\right)+ \\
& \omega_{r} \Phi_{m} F_{r d q_{x}} \\
& v_{d q_{x}}=R_{s} i_{d q_{x}}+ \\
& \left(L_{s}-M_{s}\right)\left(\frac{d i_{d q_{x}}}{d t}+i_{d q_{x}} \omega_{r}\left(\frac{1}{a_{x} e^{j \theta_{x}}}\left(e^{j \theta_{x}} \frac{d a_{x}}{d \theta_{r}}+a_{x} \frac{d e^{j \theta_{x}}}{d \theta_{r}}\right)+j\right)\right)+ \\
& \omega_{r} \Phi_{m} F_{r d q_{x}} \\
& v_{d q_{x}}=R_{s} i_{d q_{x}}+ \\
& \left(L_{s}-M_{s}\right)\left(\frac{d i_{d q_{x}}}{d t}+i_{d q_{x}} \omega_{r}\left(\frac{1}{a_{x} e^{j \theta_{x}}}\left(e^{j \theta_{x}} \frac{d a_{x}}{d \theta_{r}}+j a_{x} e^{j \theta_{x}} \frac{d \theta_{x}}{d \theta_{r}}\right)+j\right)\right)+ \\
& \omega_{r} \Phi_{m} F_{r d q_{x}} \\
& v_{d q_{x}}=R_{s} i_{d q_{x}}+ \\
& \left(L_{s}-M_{s}\right)\left(\frac{d i_{d q_{x}}}{d t}+i_{d q_{x}} \omega_{r}\left(\frac{1}{a_{x}} \frac{d a_{x}}{d \theta_{r}}+j \frac{d \theta_{x}}{d \theta_{r}}+j\right)\right)+ \\
& \omega_{r} \Phi_{m} F_{r d q_{x}} \\
& \begin{array}{c}
v_{d q_{x}}=R_{s} i_{d q_{x}}+\left(L_{s}-M_{s}\right)\left(\frac{d}{d t} i_{d q_{x}}+\omega_{r} i_{d q_{x}}\left(\frac{1}{a_{x}} \frac{d a_{x}}{d \theta_{r}}+j\left(1+\frac{d \theta_{x}}{\theta_{r}}\right)\right)\right)+ \\
\omega_{r} \Phi_{m} F_{r d q_{x}}
\end{array}
\end{aligned}
$$

Para as equações 3.12, 3.13 e 3.14, considera-se a transformação de coordenadas:

$$
\begin{gathered}
F_{r \alpha \beta}=c_{x} e^{j \theta_{r}} F_{r d q_{x}} \\
F_{r \alpha \beta}=a_{x} e^{j \theta_{x}} e^{j \theta_{r}} F_{r d q_{x}}
\end{gathered}
$$


Dessa forma:

$$
\left|F_{r \alpha \beta}\right| e^{j \theta_{F_{r \alpha \beta}}}=a_{x}\left|F_{r d q_{x}}\right| e^{j\left(\theta_{x}+\theta_{r}+\theta_{F_{r d q x}}\right)}
$$

Como $F_{r d}=0$, então $\theta_{F_{r d q x}}=\frac{\pi}{2}$. Para o ângulo da equação acima, tem-se:

$$
\theta_{F_{r \alpha \beta}}=\theta_{x}+\theta_{r}+\frac{\pi}{2}
$$

Sendo $\theta_{F_{r \alpha \beta}}=\arctan \frac{F_{r \beta}}{F_{r \alpha}}$, então:

$$
\begin{gathered}
\theta_{x}=\arctan \frac{F_{r \beta}}{F_{r \alpha}}-\frac{\pi}{2}-\theta_{r} \\
\theta_{x}=\arctan \frac{-F_{r \alpha}}{F_{r \beta}}-\theta_{r}
\end{gathered}
$$

Como o termo $F_{r d_{x}}$ é nulo, a equação 3.9 simplifica-se para a equação D.25.

$$
T_{e l}=z_{p} \Phi_{m} a_{x}^{2} F_{r q_{x}} i_{q_{x}}
$$

Comparando-se a equação do torque eletromagnético nos eixos $d q_{x}$, mostrada na equação D.25, com a equação obtida para uma MSIP com FEM senoidal, equação C.17, mostradas na equação D.26, impõe-se que $a_{x}^{2} F_{r q_{x}}=\sqrt{\frac{3}{2}}$ (equação 3.13$)^{1}$.

$$
\begin{aligned}
& T_{e l}=z_{p} \Phi_{m}\left(a_{x}^{2} F_{r q_{x}}\right) i_{q_{x}} \\
& T_{e l}=z_{p} \Phi_{m}\left(\sqrt{\frac{3}{2}}\right) i_{q}
\end{aligned}
$$

Sendo $F_{r \alpha}^{2}+F_{r \beta}^{2}=a_{x}^{2}\left(F_{r d_{x}}^{2}+F_{r q_{x}}^{2}\right)$ (relação tirada da transformação $d q_{x}$ ), e que $F_{d_{x}}=0$ (por imposição), então:

\footnotetext{
${ }^{1}$ embora no caso senoidal, utiliza-se $F_{r d}$ e não $F_{r d_{x}}$, o valor $\sqrt{\frac{3}{2}}$ foi escolhido para que a equação fosse semelhante à do caso senoidal, poderia-se ter escolhido qualquer outro valor constante.
} 


$$
\begin{gathered}
a_{x}^{2} F_{r q_{x}}^{2}=F_{r \alpha}^{2}+F_{r \beta}^{2} \\
a_{x} F_{r q_{x}}=\sqrt{F_{r \alpha}^{2}+F_{r \beta}^{2}}
\end{gathered}
$$

Substituindo a equação acima em $a_{x}^{2} F_{r q_{x}}=\sqrt{\frac{3}{2}}$, tem-se:

$$
a_{x}\left(\sqrt{F_{r \alpha}^{2}+F_{r \beta}^{2}}\right)=\sqrt{\frac{3}{2}}
$$

E, finalmente, tem-se a equação 3.14 na equação D.30:

$$
a_{x}=\sqrt{\frac{3}{2}} \frac{1}{\sqrt{F_{r \alpha}^{2}+F_{r \beta}^{2}}}
$$

Com os resultados acima, simplifica-se as equações 3.10 e 3.9 nas equações 3.15 , 3.16 e 3.17 :

$$
\begin{gathered}
v_{d_{x}}=R_{s} i_{d_{x}}+\left(L_{s}-M_{s}\right)\left(\frac{d}{d t} i_{d_{x}}+\omega_{r} i_{d_{x}} \frac{1}{a_{x}} \frac{d a_{x}}{d \theta_{r}}-\omega_{r} i_{q_{x}}\left(1+\frac{d \theta_{x}}{\theta_{r}}\right)\right)+ \\
\omega_{r} \Phi_{m} F_{r d_{x}} \\
v_{q_{x}}=R_{s} i_{q_{x}}+\left(L_{s}-M_{s}\right)\left(\frac{d}{d t} i_{q_{x}}+\omega_{r} i_{q_{x}} \frac{1}{a_{x}} \frac{d a_{x}}{d \theta_{r}}+\omega_{r} i_{d_{x}}\left(1+\frac{d \theta_{x}}{\theta_{r}}\right)\right)+ \\
\omega_{r} \Phi_{m} F_{r q_{x}}
\end{gathered}
$$

Como $F_{r d_{x}}=0$ e $F_{r q_{x}}=\sqrt{\frac{3}{2}} \frac{1}{a_{x}^{2}}$, então:

$$
v_{d_{x}}=R_{s} i_{d_{x}}+\left(L_{s}-M_{s}\right)\left(\frac{d}{d t} i_{d_{x}}+\omega_{r} i_{d_{x}} \frac{1}{a_{x}} \frac{d a_{x}}{d \theta_{r}}-\omega_{r} i_{q_{x}}\left(1+\frac{d \theta_{x}}{\theta_{r}}\right)\right)
$$




$$
\begin{gathered}
v_{q_{x}}=R_{s} i_{q_{x}}+\left(L_{s}-M_{s}\right)\left(\frac{d}{d t} i_{q_{x}}+\omega_{r} i_{q_{x}} \frac{1}{a_{x}} \frac{d a_{x}}{d \theta_{r}}+\omega_{r} i_{d_{x}}\left(1+\frac{d \theta_{x}}{\theta_{r}}\right)\right)+ \\
\sqrt{\frac{3}{2}} \Phi_{m} \frac{1}{a_{x}^{2}} \omega_{r}
\end{gathered}
$$

Para as equações 3.24 e 3.25 tem-se:

$$
T_{e l}=z_{p} \Phi_{m}\left(\sqrt{\frac{3}{2}}\right) i_{q_{x}}
$$

Então:

$$
i_{q_{x} r e f}=i_{q_{x}}=T_{\text {elref }} \sqrt{\frac{3}{2}} \frac{1}{z_{p} \Phi_{m}}
$$

E como $i_{d_{x}}=k_{i x} i_{q_{x}}=k_{i x} i_{q_{x r e f}}$ e em regime permanente, $\frac{d i_{d_{x}}}{d t}$ e $\frac{d i_{q_{x}}}{d t}$ são nulos:

$$
\begin{gathered}
v_{d_{x}}=R_{s} k_{i x} i_{q_{x} r e f}+\left(L_{s}-M_{s}\right)\left(\omega_{r} k_{i x} i_{q_{x} r e f} \frac{1}{a_{x}} \frac{d a_{x}}{d \theta_{r}}-\omega_{r} i_{q_{x} r e f}\left(1+\frac{d \theta_{x}}{\theta_{r}}\right)\right) \\
v_{q_{x}}=R_{s} i_{q_{x} r e f}+\left(L_{s}-M_{s}\right)\left(\frac{d}{d t} i_{q_{x} r e f}+\omega_{r} i_{q_{x} r e f} \frac{1}{a_{x}} \frac{d a_{x}}{d \theta_{r}}+\omega_{r} k_{i x} i_{q_{x} r e f}\left(1+\frac{d \theta_{x}}{\theta_{r}}\right)\right)+ \\
\sqrt{\frac{3}{2}} \Phi_{m} \frac{1}{a_{x}^{2}} \omega_{r}
\end{gathered}
$$

$$
v_{d_{x}}=\left(R_{s} k_{i x}+\left(L_{s}-M_{s}\right) \omega_{r}\left(\frac{1}{a_{x}} \frac{d a_{x}}{d \theta_{r}} k_{i x}-\left(1+\frac{d \theta_{x}}{\theta_{r}}\right)\right)\right) i_{q_{x} r e f}
$$




$$
\begin{gathered}
v_{q_{x}}=\left(R_{s}+\left(L_{s}-M_{s}\right) \omega_{r}\left(\frac{1}{a_{x}} \frac{d a_{x}}{d \theta_{r}}+\left(1+\frac{d \theta_{x}}{\theta_{r}}\right) k_{i x}\right)\right) i_{q_{x} r e f}+ \\
\sqrt{\frac{3}{2}} \Phi_{m} \frac{1}{a_{x}^{2}} \omega_{r}
\end{gathered}
$$

\section{D.2 Dedução das equações apresentadas no apêndice $\mathrm{C}$}

Dedução das equações C.6, C.7 e C.8 a partir das equações 3.1 e 3.2.

A partir da equação B.10 (D.41) ou B.9 (D.40):

$$
\left[\begin{array}{c}
v_{a} \\
v_{b} \\
v_{c}
\end{array}\right]=R_{s}\left[\begin{array}{c}
i_{a} \\
i_{b} \\
i_{c}
\end{array}\right]+L \frac{d}{d t}\left[\begin{array}{c}
i_{a} \\
i_{b} \\
i_{c}
\end{array}\right]+\left[\begin{array}{c}
e_{a} \\
e_{b} \\
e_{c}
\end{array}\right]+\left[\begin{array}{c}
v_{n} \\
v_{n} \\
v_{n}
\end{array}\right]
$$

Ou:

$$
\left[\begin{array}{c}
v_{a} \\
v_{b} \\
v_{c}
\end{array}\right]=R_{s}\left[\begin{array}{c}
i_{a} \\
i_{b} \\
i_{c}
\end{array}\right]+L \frac{d}{d t}\left[\begin{array}{c}
i_{a} \\
i_{b} \\
i_{c}
\end{array}\right]+\omega_{r} \Phi_{m}\left[\begin{array}{c}
F_{r a} \\
F_{r b} \\
F_{r c}
\end{array}\right]+\left[\begin{array}{c}
v_{n} \\
v_{n} \\
v_{n}
\end{array}\right]
$$

Aplicando-se a transformação $\alpha \beta 0$ :

$T\left[\begin{array}{c}v_{\alpha} \\ v_{\beta} \\ v_{0}\end{array}\right]=R_{s} T\left[\begin{array}{c}i_{\alpha} \\ i_{\beta} \\ i_{0}\end{array}\right]+L \frac{d}{d t}\left(T\left[\begin{array}{c}i_{\alpha} \\ i_{\beta} \\ i_{0}\end{array}\right]\right)+\omega_{r} \Phi_{m} T\left[\begin{array}{c}F_{r \alpha} \\ F_{r \beta} \\ F_{r 0}\end{array}\right]+\left[\begin{array}{c}v_{n} \\ v_{n} \\ v_{n}\end{array}\right]$

$T\left[\begin{array}{c}v_{\alpha} \\ v_{\beta} \\ v_{0}\end{array}\right]=R_{s} T\left[\begin{array}{c}i_{\alpha} \\ i_{\beta} \\ i_{0}\end{array}\right]+(L \cdot T) \frac{d}{d t}\left[\begin{array}{c}i_{\alpha} \\ i_{\beta} \\ i_{0}\end{array}\right]+\omega_{r} \Phi_{m} T\left[\begin{array}{c}F_{r \alpha} \\ F_{r \beta} \\ F_{r 0}\end{array}\right]+\left[\begin{array}{c}v_{n} \\ v_{n} \\ v_{n}\end{array}\right]$

Multiplicando-se ambos os lados da equação pela matriz inversa da transformação 
$\alpha \beta 0\left(T^{-1}\right)$

$$
\begin{gathered}
T^{-1} T\left[\begin{array}{c}
v_{\alpha} \\
v_{\beta} \\
v_{0}
\end{array}\right]=R_{s} T^{-1} T\left[\begin{array}{c}
i_{\alpha} \\
i_{\beta} \\
i_{0}
\end{array}\right]+T^{-1}(L \cdot T) \frac{d}{d t}\left[\begin{array}{c}
i_{\alpha} \\
i_{\beta} \\
i_{0}
\end{array}\right]+ \\
\omega_{r} \Phi_{m} T^{-1} T\left[\begin{array}{c}
F_{r \alpha} \\
F_{r \beta} \\
F_{r 0}
\end{array}\right]+T^{-1}\left[\begin{array}{c}
v_{n} \\
v_{n} \\
v_{n}
\end{array}\right]
\end{gathered}
$$

Sendo que:

$$
L \cdot T=\sqrt{\frac{2}{3}}\left[\begin{array}{ccc}
L_{s}-M_{s} & 0 & \frac{\sqrt{2}}{2}\left(L_{s}+2 M_{s}\right) \\
-\frac{1}{2}\left(L_{s}-M_{s}\right) & \frac{\sqrt{3}}{2}\left(L_{s}-M_{s}\right) & \frac{\sqrt{2}}{2}\left(L_{s}+2 M_{s}\right) \\
-\frac{1}{2}\left(L_{s}-M_{s}\right) & -\frac{\sqrt{3}}{2}\left(L_{s}-M_{s}\right) & \frac{\sqrt{2}}{2}\left(L_{s}+2 M_{s}\right)
\end{array}\right]
$$

E:

$$
\begin{aligned}
& T^{-1} \cdot(L \cdot T)=\sqrt{\frac{2}{3}}\left[\begin{array}{ccc}
1 & \frac{-1}{2} & \frac{-1}{2} \\
0 & \frac{\sqrt{3}}{2} & -\frac{\sqrt{3}}{2} \\
\frac{\sqrt{2}}{2} & \frac{\sqrt{2}}{2} & \frac{\sqrt{2}}{2}
\end{array}\right] \cdot(L \cdot T) \\
& T^{-1} \cdot(L \cdot T)=\left[\begin{array}{ccc}
L_{s}-M_{s} & 0 & 0 \\
0 & L_{s}-M_{s} & 0 \\
0 & 0 & L_{s}+2 M_{s}
\end{array}\right]
\end{aligned}
$$

Portanto: 


$$
\begin{gathered}
{\left[\begin{array}{c}
v_{\alpha} \\
v_{\beta} \\
v_{0}
\end{array}\right]=R_{s}\left[\begin{array}{c}
i_{\alpha} \\
i_{\beta} \\
i_{0}
\end{array}\right]+\left[\begin{array}{ccc}
L_{s}-M_{s} & 0 & 0 \\
0 & L_{s}-M_{s} & 0 \\
0 & 0 & L_{s}+2 M_{s}
\end{array}\right] \frac{d}{d t}\left[\begin{array}{c}
i_{\alpha} \\
i_{\beta} \\
i_{0}
\end{array}\right]+} \\
\omega_{r} \Phi_{m}\left[\begin{array}{c}
F_{r \alpha} \\
F_{r \beta} \\
F_{r 0}
\end{array}\right]+\left[\begin{array}{c}
0 \\
0 \\
\sqrt{3}
\end{array}\right] v_{n}
\end{gathered}
$$

$\mathrm{Ou}$, na forma complexa, com $v_{0}$ escrito separadamente:

$$
\begin{gathered}
v_{\alpha \beta}=R_{s} i_{\alpha \beta}+\left(L_{s}-M_{s}\right) \frac{d}{d t} i_{\alpha \beta}+\omega_{r} \Phi_{m} F_{r \alpha \beta} \\
v_{0}=R_{s} i_{0}+\left(L_{s}+2 M_{s}\right) \frac{d}{d t} i_{0}+\omega_{r} \Phi_{m} F_{r 0}+\sqrt{3} v_{n}
\end{gathered}
$$

Para a equação do torque eletromagnético, escrita nos eixos $\alpha \beta 0$, a equação C.8, parte-se da equação do torque eletromagnético por fase, equação 3.2.

$$
\begin{gathered}
T_{e l}=z_{p} \Phi_{m}\left[\begin{array}{c}
i_{a} \\
i_{b} \\
i_{c}
\end{array}\right]^{t}\left[\begin{array}{c}
F_{r a} \\
F_{r b} \\
F_{r c}
\end{array}\right] \\
T_{e l}=z_{p} \Phi_{m}\left(T\left[\begin{array}{c}
i_{\alpha} \\
i_{\beta} \\
i_{0}
\end{array}\right]^{t}\right)\left(\left[\begin{array}{c}
F_{r \alpha} \\
F_{r \beta} \\
F_{r 0}
\end{array}\right]\right)
\end{gathered}
$$

Como $T^{t}=T^{-1}$ :

$$
T_{e l}=z_{p} \Phi_{m} T^{-1} T\left[\begin{array}{c}
i_{\alpha} \\
i_{\beta} \\
i_{0}
\end{array}\right]^{t}\left[\begin{array}{c}
F_{r \alpha} \\
F_{r \beta} \\
F_{r 0}
\end{array}\right]
$$




$$
T_{e l}=z_{p} \Phi_{m}\left[\begin{array}{c}
i_{\alpha} \\
i_{\beta} \\
i_{0}
\end{array}\right]^{t}\left[\begin{array}{c}
F_{r \alpha} \\
F_{r \beta} \\
F_{r 0}
\end{array}\right]
$$

$\mathrm{Ou}:$

$$
T_{e l}=z_{p} \Phi_{m}\left(F_{r \alpha} i_{\alpha}+F_{r \beta} i_{\beta}+F_{r 0} i_{0}\right)
$$

A equação C.11 é obtida a partir da equação C.9:

$$
\begin{gathered}
T_{e l}=z_{p} \Phi_{m} \operatorname{Re}\left(F_{r \alpha \beta} i_{\alpha \beta}^{*}\right) \\
T_{e l}=z_{p} \Phi_{m} \operatorname{Re}\left(\left(e^{j \theta_{r}} F_{r d q}\right)\left(e^{j \theta_{r}} i_{d q}\right)^{*}\right)=z_{p} \Phi_{m} \operatorname{Re}\left(e^{j \theta_{r}} F_{r d q} e^{-j \theta_{r}} i_{d q}^{*}\right)
\end{gathered}
$$

Portanto:

$$
\begin{gathered}
T_{e l}=z_{p} \Phi_{m} \operatorname{Re}\left(F_{r d q} i_{d q}^{*}\right) \\
T_{e l}=z_{p} \Phi_{m}\left(F_{r d} i_{d}+F_{r q} i_{q}\right)
\end{gathered}
$$

A equação C.10 é obtida a partir da equação C.6:

$$
v_{\alpha \beta}=R_{s} i_{\alpha \beta}+\left(L_{s}-M_{s}\right) \frac{d}{d t} i_{\alpha \beta}+\omega_{r} \Phi_{m} F_{r \alpha \beta}
$$

Se $x_{\alpha \beta}=e^{j \theta_{r}} x_{d q}$, então:

$$
e^{j \theta_{r}} v_{d q}=R_{s} e^{j \theta_{r}} i_{d q}+\left(L_{s}-M_{s}\right) \frac{d}{d t}\left(e^{j \theta_{r}} i_{d q}\right)+\omega_{r} \Phi_{m} e^{j \theta_{r}} F_{r d q}
$$




$$
\begin{gathered}
v_{d q}=R_{s} i_{d q}+\left(L_{s}-M_{s}\right) e^{-j \theta_{r}}\left(e^{j \theta_{r}} \frac{d i_{d q}}{d t}+j i_{d q} \frac{d \theta_{r}}{d t} e^{j \theta_{r}}\right)+\omega_{r} \Phi_{m} F_{r d q} \\
v_{d q}=R_{s} i_{d q}+\left(L_{s}-M_{s}\right)\left(\frac{d i_{d q}}{d t}+j i_{d q} \omega_{r}\right)+\omega_{r} \Phi_{m} F_{r d q}
\end{gathered}
$$

As equações C.13 são obtidas a partir das equações de fluxo magnético, equações C.12:

$$
\begin{aligned}
& \Phi_{r a}=\Phi_{m} \cos \left(\theta_{r}\right) \\
& \Phi_{r b}=\Phi_{m} \cos \left(\theta_{r}-\frac{2 \pi}{3}\right) \\
& \Phi_{r c}=\Phi_{m} \cos \left(\theta_{r}+\frac{2 \pi}{3}\right)
\end{aligned}
$$

Da equação B.7 segue que:

$$
\begin{aligned}
{\left[\begin{array}{c}
F_{r a} \\
F_{r b} \\
F_{r c}
\end{array}\right]=\frac{1}{\Phi_{m}} \frac{d}{d \theta_{r}}\left[\begin{array}{c}
\Phi_{r a} \\
\Phi_{r b} \\
\Phi_{r c}
\end{array}\right]=\frac{d}{d \theta_{r}}\left[\begin{array}{c}
\cos \left(\theta_{r}\right) \\
\cos \left(\theta_{r}-\frac{2 \pi}{3}\right) \\
\cos \left(\theta_{r}+\frac{2 \pi}{3}\right)
\end{array}\right] } \\
F_{r a}=-\operatorname{sen}\left(\theta_{r}\right) \\
F_{r b}=-\operatorname{sen}\left(\theta_{r}-\frac{2 \pi}{3}\right) \\
F_{r c}=-\operatorname{sen}\left(\theta_{r}+\frac{2 \pi}{3}\right)
\end{aligned}
$$

E a equação C.14:

$$
F_{r \alpha \beta}=\sqrt{\frac{2}{3}}\left[\begin{array}{lll}
1 & e^{j \frac{2 \pi}{3}} & e^{-j \frac{2 \pi}{3}}
\end{array}\right]\left[\begin{array}{c}
F_{r a} \\
F_{r b} \\
F_{r c}
\end{array}\right]
$$




$$
\begin{aligned}
& F_{r \alpha \beta}=\sqrt{\frac{2}{3}}\left[\begin{array}{lll}
1 & e^{j \frac{2 \pi}{3}} & e^{-j \frac{2 \pi}{3}}
\end{array}\right]\left[\begin{array}{c}
-\frac{e^{j \theta_{r}}-e^{-j \theta_{r}}}{2 j} \\
-\frac{e^{j\left(\theta_{r}-\frac{2 \pi}{3}\right)}-e^{-j\left(\theta_{r}-\frac{2 \pi}{3}\right)}}{2 j} \\
-\frac{e^{j\left(\theta_{r}+\frac{2 \pi}{3}\right)}-e^{-j\left(\theta_{r}+\frac{2 \pi}{3}\right)}}{2 j}
\end{array}\right] \\
& F_{r \alpha \beta}=\sqrt{\frac{2}{3}}\left(-\frac{e^{j \theta_{r}}-e^{-j \theta_{r}}}{2 j}-e^{j \frac{2 \pi}{3}} \frac{e^{j\left(\theta_{r}-\frac{2 \pi}{3}\right)}-e^{-j\left(\theta_{r}-\frac{2 \pi}{3}\right)}}{2 j}\right. \\
& \left.-e^{-j \frac{2 \pi}{3}} \frac{e^{j\left(\theta_{r}+\frac{2 \pi}{3}\right)}-e^{-j\left(\theta_{r}+\frac{2 \pi}{3}\right)}}{2 j}\right) \\
& F_{r \alpha \beta}=\sqrt{\frac{2}{3}}\left(-\frac{e^{j \theta_{r}}-e^{-j \theta_{r}}}{2 j}-\frac{e^{j \theta_{r}}-e^{-j\left(\theta_{r}+\frac{2 \pi}{3}\right)}}{2 j}-\frac{e^{j \theta_{r}}-e^{-j\left(\theta_{r}-\frac{2 \pi}{3}\right)}}{2 j}\right) \\
& F_{r \alpha \beta}=\sqrt{\frac{2}{3}}\left(-\frac{e^{j \theta_{r}}}{2 j}-\frac{e^{-j \theta_{r}}}{2 j}-\frac{e^{j \theta_{r}}}{2 j}-\frac{e^{-j\left(\theta_{r}+\frac{2 \pi}{3}\right)}}{2 j}-\frac{e^{j \theta_{r}}}{2 j}-\frac{e^{-j\left(\theta_{r}-\frac{2 \pi}{3}\right)}}{2 j}\right) \\
& F_{r \alpha \beta}=\sqrt{\frac{2}{3}}\left(-\frac{e^{j \theta_{r}}}{2 j}-\frac{e^{j \theta_{r}}}{2 j}-\frac{e^{j \theta_{r}}}{2 j}-\frac{e^{-j \theta_{r}}}{2 j}-\frac{e^{-j\left(\theta_{r}+\frac{2 \pi}{3}\right)}}{2 j}-\frac{e^{-j\left(\theta_{r}-\frac{2 \pi}{3}\right)}}{2 j}\right) \\
& F_{r \alpha \beta}=\sqrt{\frac{2}{3}}\left(-3 \frac{e^{j \theta_{r}}}{2 j}-0\right) \\
& F_{r \alpha \beta}=j \sqrt{\frac{3}{2}} e^{j \theta_{r}} \\
& F_{r \alpha \beta}=e^{j \theta_{r}} F_{r d q} \\
& e^{j \theta_{r}} F_{r d q}=j \sqrt{\frac{3}{2}} e^{j \theta_{r}}
\end{aligned}
$$

Portanto: 


$$
F_{r d q}=j \sqrt{\frac{3}{2}}
$$

As equações C.15 e C.16 são desenvolvidas a partir da equação C.10. Escrevendo-se as duas partes da equação C.10 no domínio real:

$$
\begin{aligned}
& v_{d}=R_{s} i_{d}+\left(L_{s}-M_{s}\right) \frac{d i_{d}}{d t}-\left(L_{s}-M_{s}\right) i_{q} \omega_{r}+\omega_{r} \Phi_{m} F_{r d} \\
& v_{q}=R_{s} i_{q}+\left(L_{s}-M_{s}\right) \frac{d i_{q}}{d t}+\left(L_{s}-M_{s}\right) i_{d} \omega_{r}+\omega_{r} \Phi_{m} F_{r q}
\end{aligned}
$$

Como:

$$
\begin{gathered}
F_{r d}=0 \\
F_{r q}=\sqrt{\frac{3}{2}} \\
v_{d}=R_{s} i_{d}+\left(L_{s}-M_{s}\right) \frac{d i_{d}}{d t}-\left(L_{s}-M_{s}\right) i_{q} \omega_{r} \\
v_{q}=R_{s} i_{q}+\left(L_{s}-M_{s}\right) \frac{d i_{q}}{d t}+\left(L_{s}-M_{s}\right) i_{d} \omega_{r}+\sqrt{\frac{3}{2}} \omega_{r} \Phi_{m}
\end{gathered}
$$

Para a equação do torque eletromagnético, equação C.17, temos, a partir da equação C.11, com as condições D.80:

$$
\begin{gathered}
T_{e l}=z_{p} \Phi_{m}\left(i_{d} F_{r d}+i_{q} F_{r q}\right) \\
T_{e l}=z_{p} \Phi_{m} \sqrt{\frac{3}{2}} i_{q}
\end{gathered}
$$

A equação C.18 é obtida a partir da equação C.6, com a aplicação da transformação $d q$ com ângulo dos eixos $d q$ qualquer. 


$$
v_{\alpha \beta}=R_{s} i_{\alpha \beta}+\left(L_{s}-M_{s}\right) \frac{d}{d t} i_{\alpha \beta}+\omega_{r} \Phi_{m} F_{r \alpha \beta}
$$

Como $x_{\alpha \beta}=e^{j \theta_{d q}} x_{d q}$ :

$$
\begin{gathered}
e^{j \theta_{d q}} v_{d q}=R_{s} e^{j \theta_{d q}} i_{d q}+\left(L_{s}-M_{s}\right) \frac{d}{d t}\left(e^{j \theta_{d q}} i_{d q}\right)+\omega_{r} \Phi_{m} e^{j \theta_{d q}} F_{r d q} \\
v_{d q}=R_{s} i_{d q}+\left(L_{s}-M_{s}\right) e^{-j \theta_{d q}}\left(e^{j \theta_{d q}} \frac{d i_{d q}}{d t}+j i_{d q} e^{j \theta_{d q}} \frac{d \theta_{d q}}{d t}\right)+\omega_{r} \Phi_{m} F_{r d q} \\
v_{d q}=R_{s} i_{d q}+\left(L_{s}-M_{s}\right) \frac{d i_{d q}}{d t}+j\left(L_{s}-M_{s}\right) i_{d q} \frac{d \theta_{d q}}{d t}+\omega_{r} \Phi_{m} F_{r d q} \\
v_{d q}=R_{s} i_{d q}+\left(L_{s}-M_{s}\right) \frac{d i_{d q}}{d t}+j\left(L_{s}-M_{s}\right) i_{d q} \omega_{r} \frac{d \theta_{d q}}{d \theta_{r}}+\omega_{r} \Phi_{m} F_{r d q}
\end{gathered}
$$

A equação C.19 é obtida de forma semelhante à equação C.11, a partir da equação C.9:

$$
\begin{gathered}
T_{e l}=z_{p} \Phi_{m} \operatorname{Re}\left(F_{r \alpha \beta} i_{\alpha \beta}^{*}\right) \\
T_{e l}=z_{p} \Phi_{m} \operatorname{Re}\left(\left(e^{j \theta_{d q}} F_{r d q}\right)\left(e^{j \theta_{d q}} i_{d q}\right)^{*}\right)=z_{p} \Phi_{m} \operatorname{Re}\left(e^{j \theta_{d q}} F_{r d q} e^{-j \theta_{d q}} i_{d q}^{*}\right)
\end{gathered}
$$

Portanto:

$$
T_{e l}=z_{p} \Phi_{m} \operatorname{Re}\left(F_{r d q} i_{d q}^{*}\right)
$$




$$
T_{e l}=z_{p} \Phi_{m}\left(F_{r d} i_{d}+F_{r q} i_{q}\right)
$$

A equação C.20 é obtida da transformação $d q$ :

$$
\begin{gathered}
F_{r \alpha \beta}=e^{j \theta_{d q}} F_{r d q} \\
\left|F_{r \alpha \beta}\right| e^{j \theta_{F_{r \alpha \beta}}}=\left|F_{r d q}\right| e^{j\left(\theta_{F_{r d q}}+\theta_{d q}\right)}
\end{gathered}
$$

Onde:

$$
\begin{aligned}
& \theta_{F_{r \alpha \beta}}: \text { ângulo de } F_{r \alpha \beta} ; \\
& \theta_{F_{r d q}}: \text { ângulo de } F_{r d q} .
\end{aligned}
$$

Então:

$$
\left|F_{r \alpha \beta}\right|=\left|F_{r d q}\right|
$$

Ou seja:

$$
F_{r \alpha}^{2}+F_{r \beta}^{2}=F_{r d}^{2}+F_{r q}^{2}
$$

Como $F_{r d}=0$ :

$$
F_{r q}=\sqrt{F_{r \alpha}^{2}+F_{r \beta}^{2}}
$$

Para a equação C.21, utiliza-se da equação D.95:

$$
\theta_{F_{r \alpha \beta}}=\theta_{F_{r d q}}+\theta_{d q}=\arctan \frac{F_{r \beta}}{F_{r \alpha}}
$$

Como $\theta_{F_{r d q}}=\frac{\pi}{2}$, pois $F_{r d}=0$ :

$$
\arctan \frac{F_{r \beta}}{F_{r \alpha}}=\frac{\pi}{2}+\theta_{d q}
$$




$$
\theta_{d q}=\arctan \frac{-F_{r \alpha}}{F_{r \beta}}
$$

As equações C.22 e C.23 são obtidas a partir das equações C.10, C.20 e C.21:

$$
\begin{aligned}
& v_{d}=R_{s} i_{d}+\left(L_{s}-M_{s}\right) \frac{d}{d t} i_{d}-\left(L_{s}-M_{s}\right) \omega_{r} \frac{d \theta_{d q}}{d \theta_{r}} i_{q}+\omega_{r} \Phi_{m} F_{r d} \\
& v_{q}=R_{s} i_{q}+\left(L_{s}-M_{s}\right) \frac{d}{d t} i_{q}+\left(L_{s}-M_{s}\right) \omega_{r} \frac{d \theta_{d q}}{d \theta_{r}} i_{d}+\omega_{r} \Phi_{m} F_{r q}
\end{aligned}
$$

Como $F_{r d}=0$ :

$$
v_{d}=R_{s} i_{d}+\left(L_{s}-M_{s}\right) \frac{d}{d t} i_{d}-\left(L_{s}-M_{s}\right) \omega_{r} \frac{d \theta_{d q}}{d \theta_{r}} i_{q}
$$

A equação C.24 é obtida a partir da equação C.19 com $F_{r d}=0$ :

$$
T_{e l}=z_{p} \Phi_{m}\left(F_{r d} i_{d}+F_{r q} i_{q}\right)
$$

$$
T_{e l}=z_{p} i_{q} \Phi_{m} F_{r q}
$$




\section{Apêndice E}

\section{Descrição Física da Máquina}

A máquina utilizada no projeto é uma máquina síncrona com ímã permanente (MSIP) na superfície do rotor fabricada pela Siemens, modelo 1FT5 062 OAC01. Comercialmente, essa máquina é vendida juntamente com seu conversor elétrico, onde o conjunto resultante é denominado de "Brushless DC Motor". O conversor elétrico dessa máquina opera no modo "seis-pulsos" clássico, com a freqüência de operação sincronizada com o rotor da máquina.

Um corte transversal da máquina síncrona considerada no projeto é visto na figura E.1, onde pode-se notar melhor sua estrutura física. A máquina possui 24 pastilhas magnéticas (4 pastilhas por pólo) e 36 ranhuras no estator (Oliveira Jr., 1990).

Através da figura, observa-se que as pastilhas magnéticas são colocadas na superfície do rotor. Nota-se também, que o rotor da máquina é oco, permitindo que esse tipo de máquina tenha um baixo momento de inércia de rotor.

Os parâmetros da máquina utilizada podem ser vistos na tabela E.1.

A máquina possui três tipos de conexões, que são:

- Conexão de alimentação das fases;

- Conexão dos sinais de controle e outras informações da máquina;

- Conexão do encoder acoplado ao eixo.

A conexão de alimentação consiste de três fios com bitola aproximadamente de 1,5 $\mathrm{mm}^{2}$. Cada fio é ligado a uma fase da máquina, sendo que o fio da conexão central não 


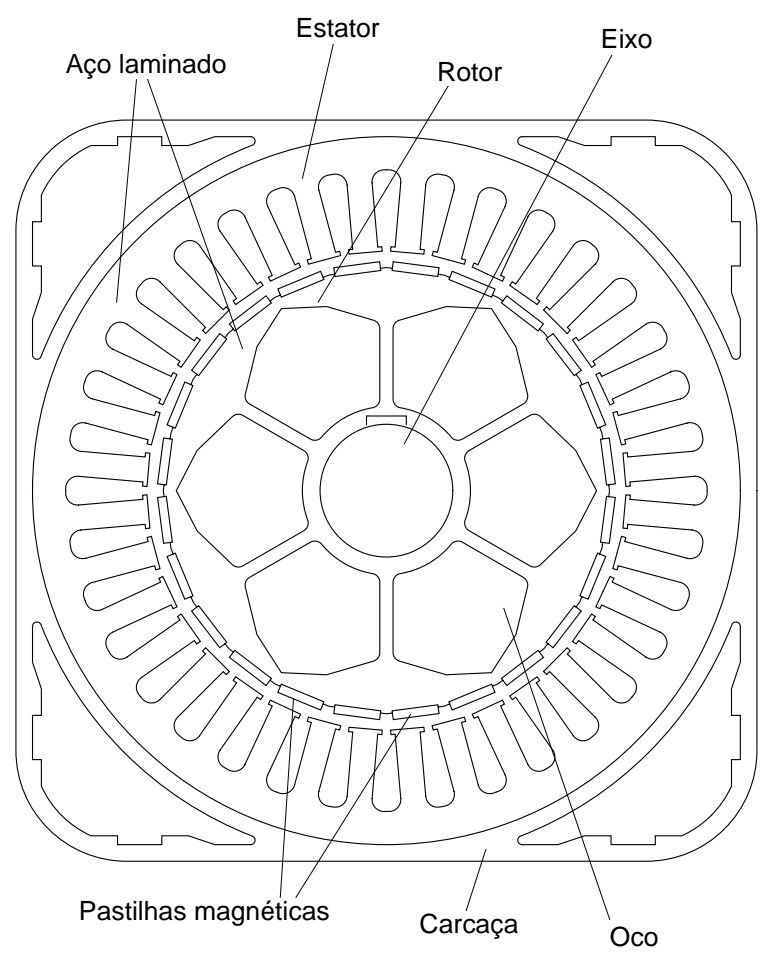

Figura E.1: Corte transversal da máquina síncrona Siemens 1FT5.

Tabela E.1: Parâmetros da máquina Siemens 1FT5 062.

\begin{tabular}{|rl|}
\hline Modelo do motor & 1FT5 062 OAC01 \\
\hline Modelo do taco-gerador & 1 FU1 050 6HC 200 \\
\hline Velocidade nominal & $2000 \mathrm{rpm}$ \\
Corrente nominal & $3,5 \mathrm{~A}$ \\
Torque nominal & $2,2 \mathrm{~N} \cdot \mathrm{m}$ \\
Tensão nominal & $150 \mathrm{~V}$ \\
Número de pólos $\left(2 \cdot z_{p}\right)$ & 6 \\
Resistência de estator $\left(R_{s}\right)$ & $2,4 \Omega$ \\
Indutância de estator $\left(L_{s}\right)$ & $12,4 \mathrm{mH}$ \\
Constante de tensão $\left(k_{e}\right)$ & $0,72 \mathrm{~V} / \mathrm{rad} / \mathrm{s}$ \\
Momento de inércia $\left(J_{m}\right)$ & $4,2 \cdot 10^{-3} \mathrm{~kg} \cdot \mathrm{m}^{2}$ \\
\hline
\end{tabular}




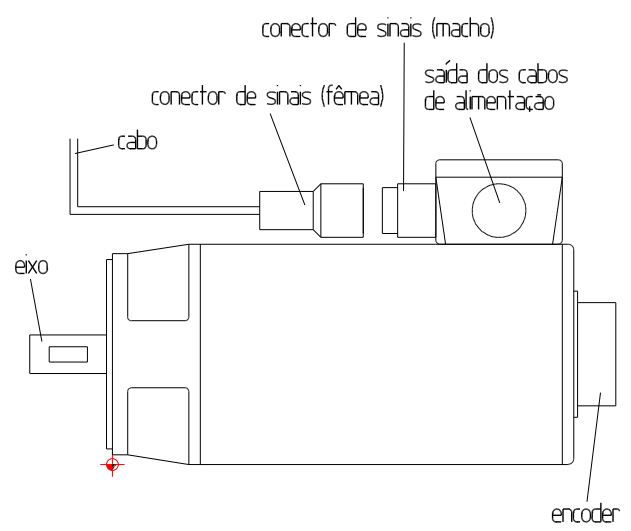

Figura E.2: Perfil da máquina 1FT5, com o detalhe do conector de sinais.

está disponível. A figura 3.1 mostra esquematicamente as ligações de alimentação da máquina. A ligação da máquina é feita em estrela (ou "Y"). A máquina da figura, por questões de simplicidade, possui somente dois pólos magnéticos no rotor; já a máquina real possui seis pólos magnéticos.

Um desenho do perfil da máquina é mostrado na figura E.2. Na figura, podem ser vistos o conector de sinais do motor, o encoder acoplado ao eixo e a saída dos cabos de alimentação da máquina.

O conector de sinais pode ser visto em maior detalhe na figura E.3. Os seguintes sinais estão disponíveis nesse conector:

- Temperatura do motor;

- Sensores de posição internos de efeito hall;

- Sinais do taco-gerador interno.

Esses sinais devem ser lidos e passados ao sistema de processamento central. O sinal da temperatura é importante em casos de sobrecarga da máquina, onde esta pode sofrer danos permanentes caso a temperatura máxima de operação seja ultrapassada.

A pinagem do conector mostrado na figura E.3 é mostrada na tabela E.2.

Os sinais produzidos pelos sensores de posição internos de efeito hall estão apresentados na figura E.4. Com esses três sinais, é possível se conhecer a posição absoluta do 


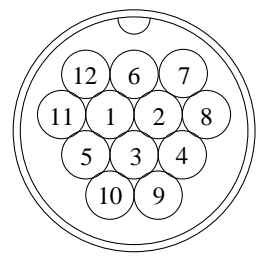

Figura E.3: Conector do motor brushless 1FT5 062 - vista traseira do conector fêmea.

Tabela E.2: Pinagem do conector de sinais.

\begin{tabular}{|c|l|}
\hline 1 & Sensor de posição $1\left(0^{\circ}\right)$ - coletor aberto; \\
2 & Sensor de posição $2\left(+120^{\circ}\right)$ - coletor aberto; \\
3 & Sensor de posição $3\left(-120^{\circ}\right)$ - coletor aberto; \\
4 e 5 & Alimentação dos sensores de posição - VCC e GND, respectivamente; \\
6 & Comum do taco-gerador; \\
7 & Taco-gerador - $120^{\circ} ;$ \\
8 & Sem conexão interna; \\
9 e 10 & Termistor PTC; \\
11 & Taco-gerador $+120^{\circ} ;$ \\
12 & Taco-gerador $0^{\circ} ;$ \\
\hline
\end{tabular}

rotor em intervalos de $60^{\circ}$ (posição em graus elétricos). Com a informação da posição absoluta do rotor dada pelos sensores internos e com os sinais do encoder, tem-se uma informação mais precisa da posição do rotor a cada instante.

A conexão do encoder foi feita posteriormente na máquina, não existindo originalmente.

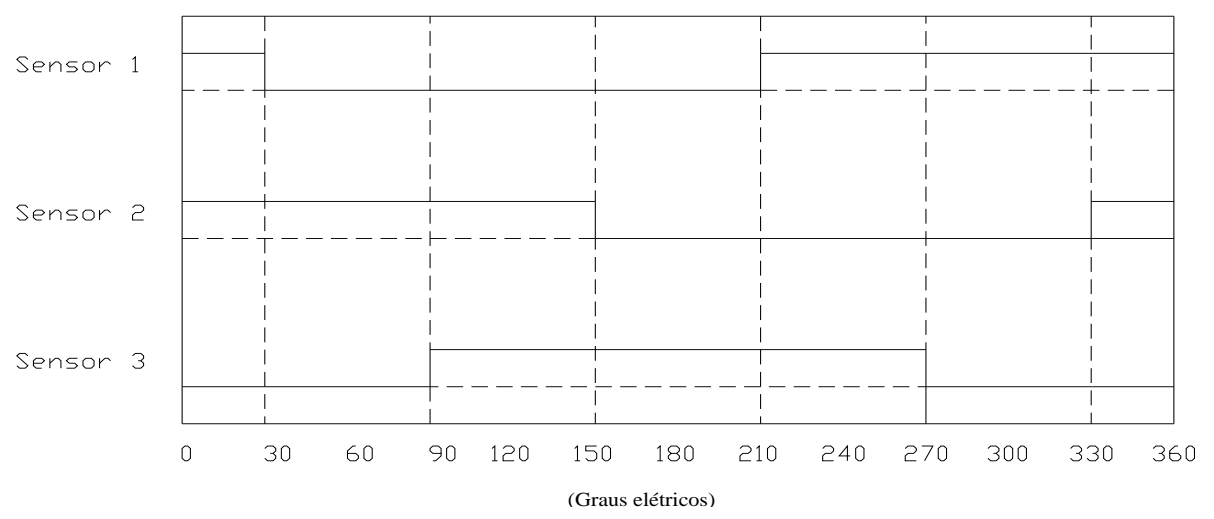

Figura E.4: Sinais dos sensores de posição internos de efeito hall. 


\section{Apêndice F}

\section{Implementação do sistema de controle}

O hardware desenvolvido pode ser dividido em três camadas principais:

- Camada de potência

- Camada de interface analógica/digital

- Camada de processamento digital

Pode-se visualizar um diagrama esquemático do hardware desenvolvido na figura F.1, onde pode-se ver as três camadas que compõem o projeto.

\section{F.1 Camada de Potência}

A camada de potência compreende o conector de entrada AC, as fontes de alimentação, que provê circuitos eletricamente isolados entre a própria camada de potência e as demais camadas, o módulo retificador e o módulo inversor.

O módulo retificador possui entrada de $220 \mathrm{~V}$ trifásico e capacidade de corrente contínua de saída de 25A. Possui um circuito de carga responsável pela carga dos capacitores do filtro DC, que possuem alta capacitância (perto de $2000 \mu \mathrm{F}$ ) para a tensão empregada. O esquema do módulo retificador é mostrado na figura F.2.

O diagrama esquemático do módulo inversor pode ser visto na figura F.3. O módulo 


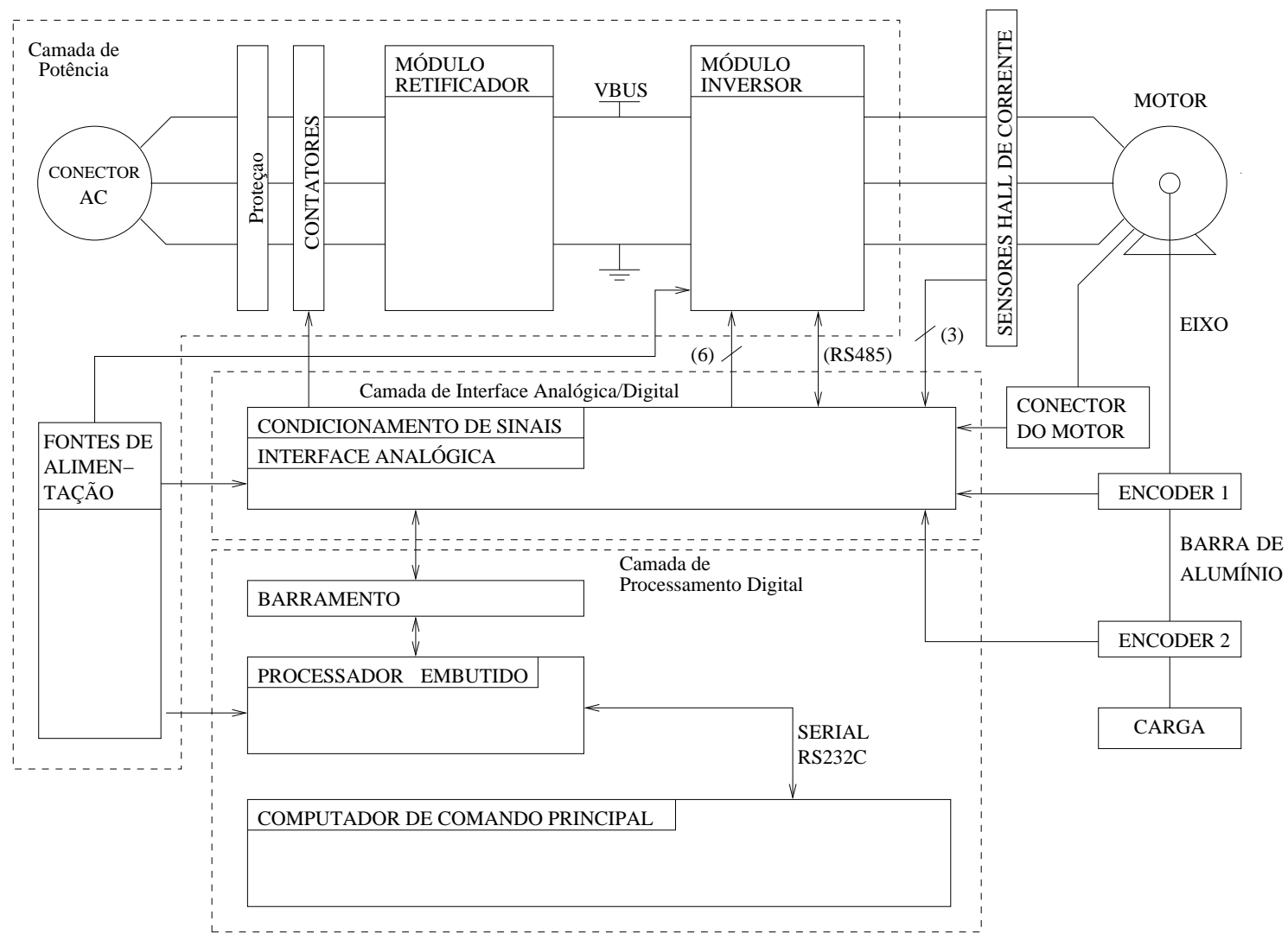

Figura F.1: Sistema de controle proposto.

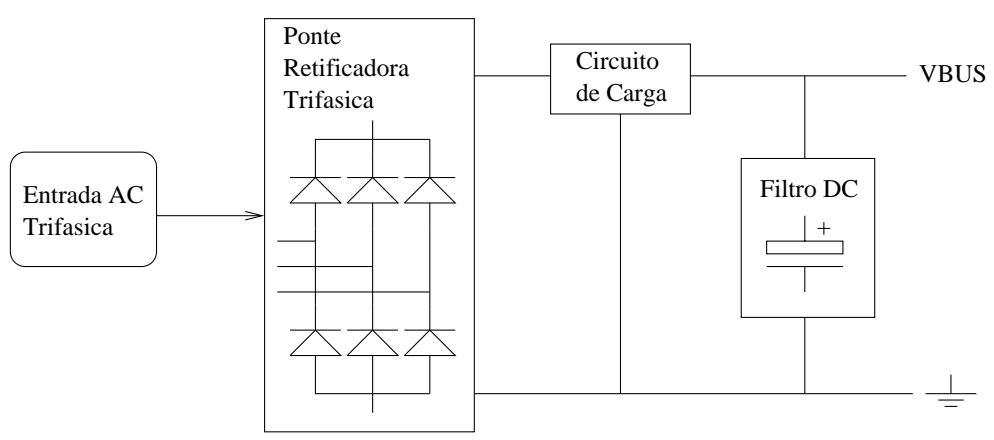

Figura F.2: Diagrama esquemático do módulo retificador. 


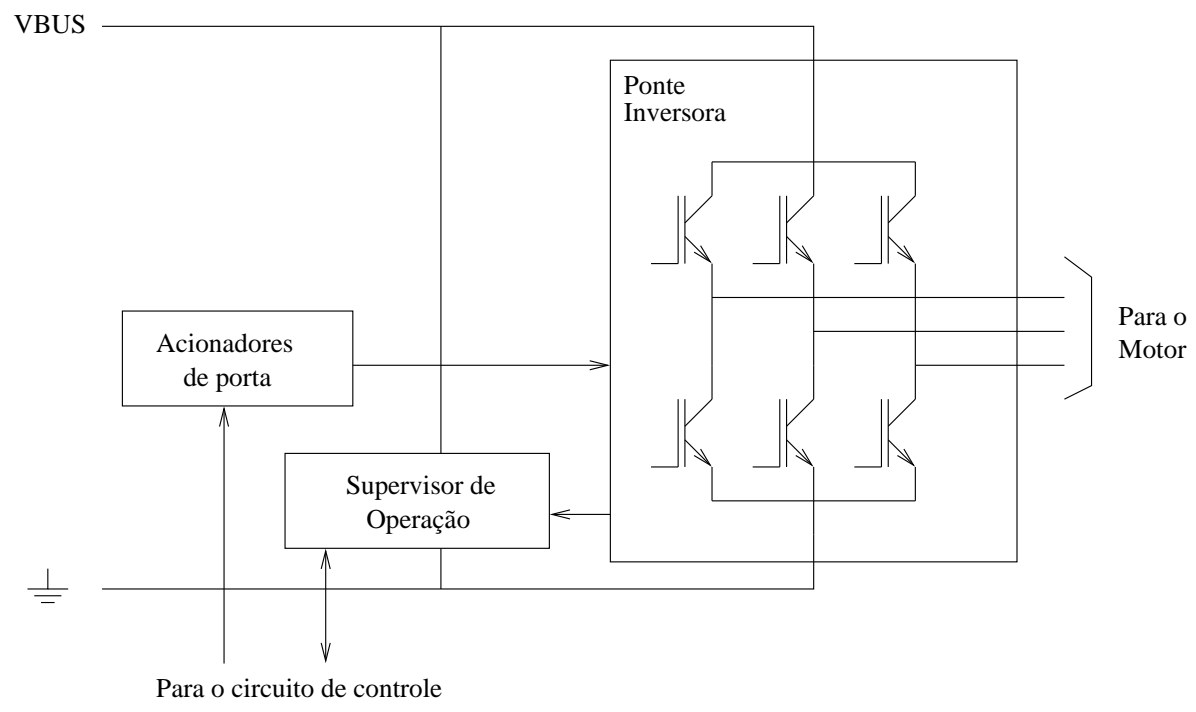

Figura F.3: Diagrama esquemático do módulo inversor.

inversor é composto basicamente por três blocos distintos: a ponte inversora, que consiste em seis transistores IGBTs de potência; o circuito de acionamento de porta, que cuida da isolação entre os sinais de controle, vindos do circuito de controle, e as portas dos transistores IGBTs, bem como das proteções de sobrecorrente e sobretensão na ponte inversora; e o circuito supervisor de operação, que monitora a corrente, a tensão, e a temperatura na ponte inversora e transmite esses dados para o circuito de controle, permitindo que o circuito de controle tome algumas decisões preventivas em caso de operações anormais. Essa monitoração de corrente é feita em um intervalo relativamente grande de tempo (da ordem de microsegundos), servindo somente para monitorar condições de sobrecarga da máquina. Para curto-circuitos que possam destruir a ponte inversora, o módulo inversor possui um laço de emergência interno (no próprio bloco "Acionadores de Porta") que desativa as chaves da ponte inversora em caso de correntes excessivas, de modo a não destruir o sistema.

\section{F.2 Camada de Interface Analógica/Digital}

A camada de interface analógica/digital permite que a camada de processamento tenha acesso a todos os sinais mostrados na figura F.1. Para isso, o circuito de interface analógica/digital possui A/Ds, saídas de relês e saídas e entradas de lógica digital.

A camada de interface analógica/digital se comunica com a camada de processa- 
Tabela F.1: Entradas e saídas da camada de interface analógica/digital. Na tabela, $\mathrm{E} / \mathrm{S}=$ entrada ou saída, $\mathrm{E}=$ entrada e $\mathrm{S}=$ saída.

\begin{tabular}{|c|c|l|c|}
\hline E/S & Tipo & Descrição/Função & Quantidade \\
\hline E & Analógica & Corrente nas fases & 3 \\
E & Digital & Sensores internos de posição do rotor & 3 \\
E & Analógica & Temperatura do motor & 1 \\
E & Analógica & Tacogerador interno do motor & 3 \\
E & Digital & Encoder (entrada em quadratura) & 2 \\
E/S & RS485 & Comunicação serial UART com o módulo inversor & 1 \\
S & Digital & Sinais das portas para o módulo inversor & 6 \\
S & Relê & Acionamento dos módulos de potência & 1 \\
& & (via contator trifásico) & \\
\hline
\end{tabular}

mento digital através de um barramento paralelo. A arquitetura desse barramento é extremamente dependente do tipo de processador adotado. Detalhes sobre o processador adotado estão apresentados na seção F.3.

A interface analógica/digital possui os seguintes elementos fundamentais:

- Conversores analógico/digital;

- Relê e acionador de relês, com isolador de ruído;

- Driver RS485;

- Drivers para saídas digitais;

- Drivers para entradas digitais.

Todos os componentes acima devem ser controlados através do barramento, pelo processador do sistema. A figura F.4 mostra um diagrama em blocos da placa analógica implementada.

Na figura, pode-se ver que o barramento é responsável pelo controle de todos os ítens expostos acima. Esse é um barramento paralelo de alta velocidade, se comparado a interfaces seriais.

O conversor analógico digital utilizado possui 6 canais diferencias de entrada e realiza 400 mil conversões por segundo. A resolução do conversor é de 12 bits, suficiente para medidas precisas. Os sinais lidos pelo conversor são os sinais de corrente, sinal de temperatura do motor, possivelmente, um sinal de temperatura da ponte inversora 


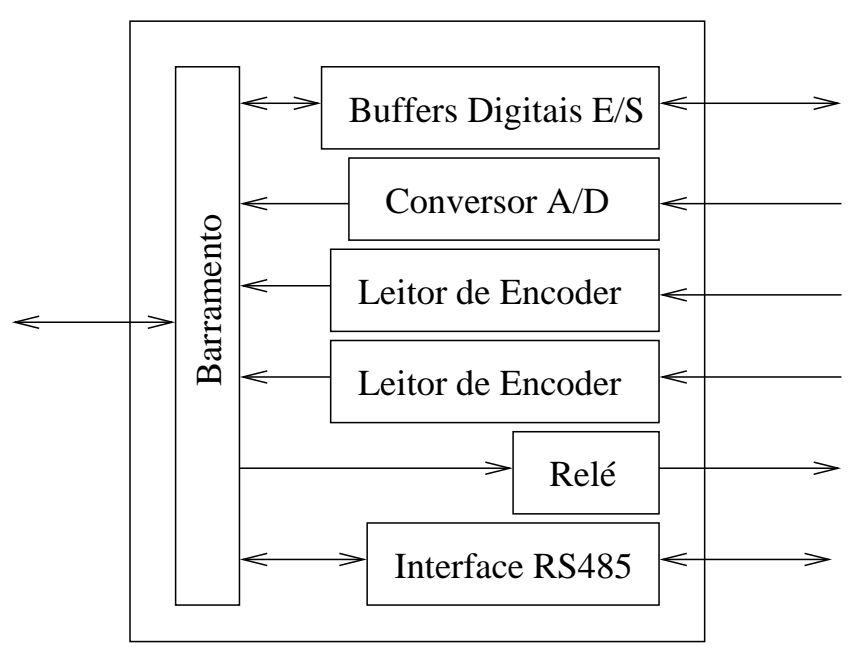

Figura F.4: Diagrama em blocos da placa de interface analógica/digital.

IGBT e os sinais vindos do tacogerador do motor. O barramento e as placas analógicas foram projetados de tal maneira que, caso mais canais analógicos venham a ser necessários, pode-se acoplar mais placas ao barramento (até quatro placas analógicas).

O relê é utilizado para acionar os contatores que alimentam o retificador e o inversor.

Os sinais de entrada e saída digitais têm como função inicial o acionamento das fases do inversor. Alguns bits de entrada e de saída foram acrescentados para posteriores necessidades.

Os dois encoders são lidos através de dois canais de interrupção do processador. O valor de cada encoder é decrementado ou incrementado de acordo com o sentido de rotação da máquina, que é sentido pelo estado do segundo canal de cada encoder. Portanto, cada encoder é ligado a um canal de interrupção e a um bit de entrada genérico. Considerou-se dois leitores de encoder a fim de possibilitar a medição do torque pela torsão da barra de alumínio.

\section{F.3 Camada de Processamento Digital}

O processador utilizado é o processador LH77790 ${ }^{1}$, fabricado pela Sharp Microelectronics $^{2}$. Esse processador trabalha com clock de $24 \mathrm{MHz}$ e, devido à sua arquitetura

\footnotetext{
${ }^{1}$ http://www. sharpmeg.com/lh77/lh77 .html

${ }^{2}$ http://www . sharpmeg.com
} 
RISC, realiza a maioria das instruções em um ciclo de clock, em uma média 22,5 MIPS (milhões de instruções por segundo).

Internamente, esse processador possui três geradores de PWM, três contadores/temporizadores configuráveis independentes. Possui instruções do tipo multiplica/acumula (utilizadas para otimizar algoritmos para processamento de sinais). No entanto, não possui conversor A/D interno e leitores de quadratura de encoder.

O compilador em linguagem $\mathrm{C}$ para esse processador é de uso livre, não acarretando gastos com software específico para o processador. Todo o sistema pode ser programado em ambiente GNU/Linux ${ }^{3}$. O ambiente GNU/Linux oferece uma maior flexibilidade e liberdade na utilização de ferramentas de programação. O compilador $\mathrm{C}$ utilizado no projeto é o compilador GNU CC, ou GCC (GNU Cross Compiler).

O autor utilizou, no desenvolvimento do projeto, ferramentas de software com licença livre (software livre). Esse fato acarretou em um primeiro momento uma redução bastante grande no custo de desenvolvimento do sistema. Como exemplo, pode-se citar que o compilador para essa arquitetura custa cerca de seis mil e quinhentos dólares (ARM DEVELOPER SUITE, v.1.0.1 - versão para MS Windows ou Unix) ${ }^{4}$.

Foi utilizada uma placa com o processador Sharp LH77790A. Essa placa possui 128kBytes de ROM-Flash, 256kBytes de RAM e uma interface RS232C. Os programas de controle serão desenvolvidos no computador (através do compilador "cross") e enviados para a placa através dessa interface RS232C.

O barramento paralelo, que interliga a placa de controle com a placa da interface analógica/digital, contém circuitos lógicos de alta velocidade, a fim de aproveitar a velocidade do processador utilizado.

A placa do processador está representada na figura F.5. Pode-se visualizar na figura, que a placa possui quatro conectores tipo "HEADER", com 50 pinos cada conector, que estão conectados com cada um dos pinos do processador. O processador possui um total de 176 pinos. Através desses conectores é que se conectará a placa do barramento. Podese ver na figura o conector DB9, da interface RS232C, responsável pela comunicação com o computador.

\footnotetext{
${ }^{3}$ http://www.gnu.org

${ }^{4}$ http://www.arm.com/sitearchitek/devtools.ns4/html/dev_tools?OpenDocument\&style=Dev_Tools
} 




Figura F.5: Placa do processador. 


\section{Apêndice G}

\section{Descrição do Programa da Placa}

\section{de Controle}

Como a máquina possui 3 pólos, cada volta foi dividida em 333,33 pulsos de encoder, isto significa 55,6 pulsos a cada $60^{\circ}$ elétricos. Simplificadamente, o algoritmo de controle utilizado na placa de controle pode ser visto na figura G.1.

Na ocorrência de uma interrupção do temporizador, que ocorre ao final de cada ciclo de PWM, o valor de $\theta_{r}$ é lido (através dos registradores do encoder) e, juntamente com o valor do torque de referência, são utilizados para o cálculo do ciclos de trabalho dos três canais de PWM.

Os valores calculados dos ciclos de trabalho somente terão efeito no próximo ciclo de PWM. Os valores do ciclo atual foram calculados no ciclo anterior de PWM. Como a freqüência de PWM utilizada foi de $5,88 \mathrm{kHz}$, o ciclo de PWM, e de cálculo, é de $170 \mu$ s.

Para o cálculo dos ciclos de PWM foram utilizadas as equações apresentadas no capítulo 3, as equações para a MSIP em regime permanente de torque eletromagnético, equações $3.23,3.24$ e 3.25 .

Mas foi necessária uma modificação nessas equações para sua utilização na placa de controle. Essas equações foram discretizadas e normalizadas a fim de que rodassem mais rápido no processador utilizado, pois operações com números inteiros são bem mais rápidas do que operações com números de ponto flutuante, considerando-se que o processador utilizado não possui unidade de ponto flutuante. Todos os valores foram 


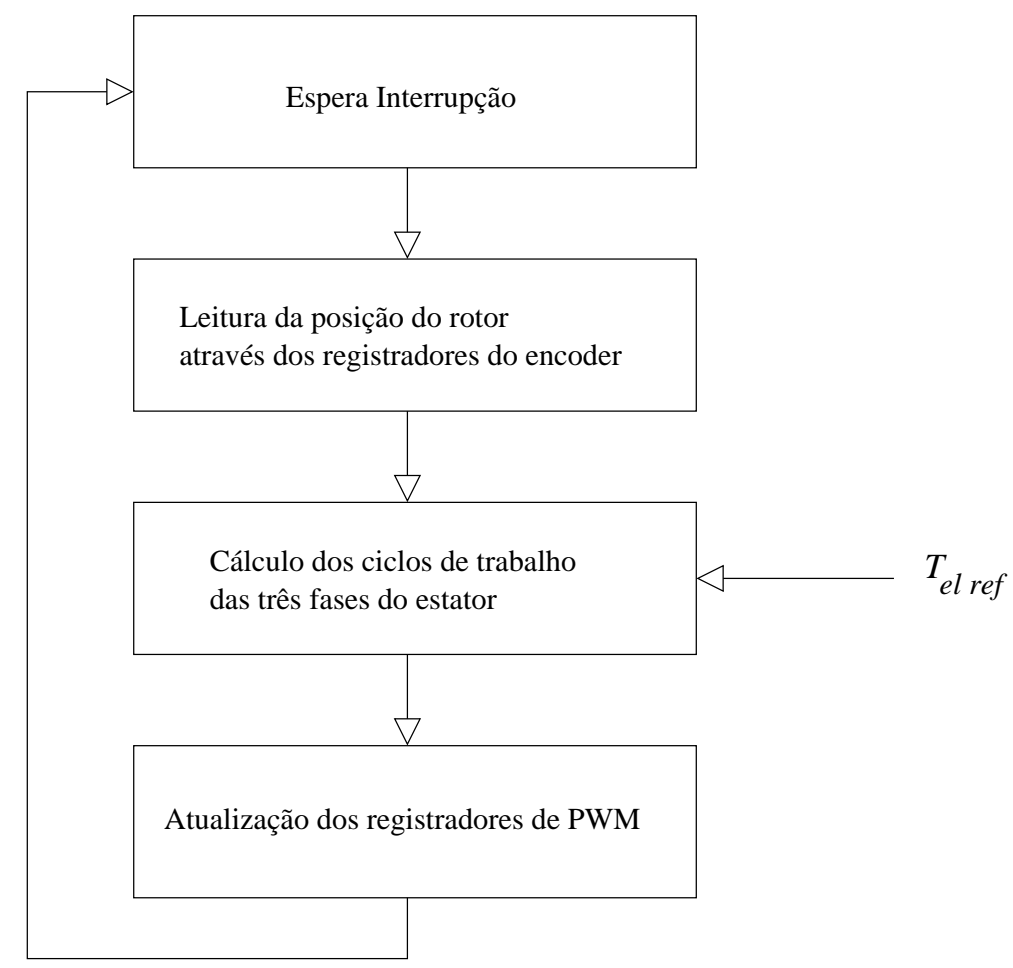

Figura G.1: Algoritmo do programa de controle em malha aberta de corrente.

normalizados para 10 bits.

Todas as funções foram tabeladas a fim de se minimizar ao máximo o número de operações do processador.

As equações utilizadas no processador para o controle vetorial da máquina estão apresentados a seguir. Nas equações, os valores normalizados são mostrados com o índice $N$.

$$
\begin{gathered}
v_{d_{x}}^{N}=\frac{\left(F_{1}\left[i_{k_{i x}}\right]+\frac{F_{2}\left[i_{k_{i x}}, \theta_{r i}\right] \stackrel{\omega}{\omega}_{r}^{N}}{2^{N}}\right){ }_{i_{q_{x}} r e f}^{N}}{2^{N}} \\
v_{q_{x}}^{N}=\frac{\left(R_{s}^{N}+\frac{F_{3}\left[i_{k_{i x}}, \theta_{r i}\right] \stackrel{\omega}{\omega}_{r}^{N}}{2^{N}}\right)_{i_{x} r e f}^{N}+\stackrel{N}{\omega}_{r}^{N} F_{4}\left[\theta_{r i}\right]}{2^{N}}
\end{gathered}
$$


Tabela G.1: Tabela para os valores de $k_{i x}$.

\begin{tabular}{|c|c|}
\hline$i_{k_{i x}}$ & $k_{i x}$ \\
\hline 0 & $-0,3$ \\
1 & $-0,2$ \\
2 & $-0,1$ \\
3 & 0 \\
4 & 0,1 \\
5 & 0,2 \\
\hline
\end{tabular}

$$
\begin{aligned}
i_{\delta a} & =\frac{F_{d a}\left[\theta_{r i}\right] v_{d_{x}}^{N}-F_{q a}\left[\theta_{r i}\right] v_{q_{x}}^{N}}{2^{N}} \\
i_{\delta b} & =\frac{F_{d b}\left[\theta_{r i}\right] v_{d_{x}}^{N}-F_{q b}\left[\theta_{r i}\right] v_{q_{x}}^{N}}{2^{N}} \\
i_{\delta b} & =\frac{F_{d c}\left[\theta_{r i}\right] v_{d_{x}}^{N}-F_{q c}\left[\theta_{r i}\right] v_{q_{x}}^{N}}{2^{N}}
\end{aligned}
$$

Onde:

$N$ : 10 bits de normalização $\left(2^{N}=1024\right)$;

$\stackrel{N}{v_{d_{x}}}, \stackrel{v_{q_{x}}}{N}, \stackrel{N}{\omega_{r}}$ e $i_{q_{x} r e f}^{N}$ : valores normalizados de $v_{d_{x}}, v_{q_{x}}, \omega_{r}$ e $i_{q_{x} r e f}$, respectivamente;

$i_{\delta a}, i_{\delta b}$ e $i_{\delta c}$ : valores discretizados dos ciclos de trabalho das fases a, b e c, respectivamente (canais 0,1 e 2 do PWM);

$\theta_{r i}$ : valor discreto de $\theta_{r}$, de 0 a 332 (333 pulsos por ciclo elétrico);

$i_{k_{i x}}$ : índice para $k_{i x}$, buscado em tabela, conforme a tabela G.1.

Os valores de $F_{1}, F_{2}, F_{3}, F_{4}, F_{d a}, F_{q a}, F_{d b}, F_{q b}, F_{d c}$ e $F_{q c}$, também são tabelados e seus elementos são calculados de acordo com as equações a seguir.

$$
\begin{gathered}
F_{1}\left[i_{k_{i x}}\right]=2^{N} R s * k_{i x}\left[i_{k_{i x}}\right] \\
F_{2}\left[i_{k_{i x}}, \theta_{r i}\right]=2^{N}\left(L_{s}-M_{s}\right)\left(k_{i x}\left[i_{k_{i x}}\right] \frac{1}{a_{x}\left(\theta_{r}\right)} \frac{d a_{x}\left(\theta_{r}\right)}{d \theta_{r}}-\left(1+\frac{d \theta_{x}\left(\theta_{r}\right)}{d \theta_{r}}\right)\right)
\end{gathered}
$$




$$
\begin{gathered}
F_{3}\left[i_{k_{i x}}, \theta_{r i}\right]=2^{N}\left(L_{s}-M_{s}\right)\left(\frac{1}{a_{x}\left(\theta_{r}\right)} \frac{d a_{x}\left(\theta_{r}\right)}{d \theta_{r}}+k_{i x}\left[i_{k_{i x}}\right]\left(1+\frac{d \theta_{x}\left(\theta_{r}\right)}{d \theta_{r}}\right)\right) \\
F_{4}\left[\theta_{r i}\right]=2^{N} \sqrt{\frac{2}{3}} \Phi_{m} \frac{1}{a_{x}\left(\theta_{r}\right)^{2}}
\end{gathered}
$$

$$
\begin{aligned}
& F_{d a}\left[\theta_{r i}\right]=2^{N} a_{x}\left(\theta_{r}\right) \sqrt{\frac{2}{3}} \cos \left(\theta_{r}+\theta_{x}\left(\theta_{r}\right)\right) \\
& F_{q a}\left[\theta_{r i}\right]=2^{N} a_{x}\left(\theta_{r}\right) \sqrt{\frac{2}{3}} \operatorname{sen}\left(\theta_{r}+\theta_{x}\left(\theta_{r}\right)\right) \\
& F_{d b}\left[\theta_{r i}\right]=2^{N} a_{x}\left(\theta_{r}\right) \sqrt{\frac{2}{3}} \cos \left(\theta_{r}+\theta_{x}\left(\theta_{r}\right)-120^{\circ}\right) \\
& F_{q b}\left[\theta_{r i}\right]=2^{N} a_{x}\left(\theta_{r}\right) \sqrt{\frac{2}{3}} \operatorname{sen}\left(\theta_{r}+\theta_{x}\left(\theta_{r}\right)-120^{\circ}\right) \\
& F_{d c}\left[\theta_{r i}\right]=2^{N} a_{x}\left(\theta_{r}\right) \sqrt{\frac{2}{3}} \cos \left(\theta_{r}+\theta_{x}\left(\theta_{r}\right)+120^{\circ}\right) \\
& F_{q c}\left[\theta_{r i}\right]=2^{N} a_{x}\left(\theta_{r}\right) \sqrt{\frac{2}{3}} \operatorname{sen}\left(\theta_{r}+\theta_{x}\left(\theta_{r}\right)+120^{\circ}\right)
\end{aligned}
$$

Nas equações, o valor discreto de $\theta_{r}$ é dado pela equação G.9.

$$
\theta_{r i}=333 \frac{\theta_{r}}{2 \pi}
$$

Para o valor de $i_{q_{x} r e f}^{N}$, temos a equação G.10, onde a normalização em 10 bits não implica em uma grande perda de precisão de $i_{q_{x} r e f}$.

$$
\stackrel{i_{q_{x} r e f}^{N}}{N}=\left(\frac{2^{N}}{z_{p} \sqrt{\frac{3}{2}} \Phi_{m}}\right) T_{\text {elref }}^{N} \frac{1}{2^{N}}
$$




\section{Parte V}

\section{Referências}




\section{Referências Bibliográficas}

(Aghili et al., 2000) Aghili, F., Buehler, M., e Hollerbach, J. M. Optimal Commutation Laws in the Frequency Domain for PM Synchronous Direct-Drive Motors. IEEE Transactions on Power Electronics, 15(6), 2000.

(Berendsen et al., 1993) Berendsen, C. S., Champenois, G., e Bolopion, A. Commutation strategies for brushless DC motors: influence on instant torque. IEEE Transaction on Power Electronics, 8(2), 1993.

(Chan et al., 1996) Chan, C. C., Chau, K. T., Jiang, J. Z., Xia, W., Zhu, M., e Zhang, R. Novel Permanent Magnet Motor Drives for Electric Vehicles. IEEE Transaction on Industrial Electronics, 43(2), 1996.

(Chan et al., 1994) Chan, C. C., Jiang, J. Z., Chen, G. H., Wang, X. Y., e Chau, K. T. A Novel Polyphase Multipole Square-Wave Permanent Magnet Motor Drives for Electric Vehicles. IEEE Transaction on Industry Applications, 30(5), 1994.

(Chung et al., 1998) Chung, S. K., Kim, H. S., Kim, C. G., e Youn, M. J. A new instantaneous torque control of PM synchronous motor for high-performance direct-drive applications. IEEE Transactions on Power Electronics, 13(3), 1998.

(French \& Acarnley, 1996) French, C. e Acarnley, P. Control of Permanent Magnet Motor Drives Using a New Position Estimation Technique. IEEE Transaction on Industry Applications, 32(5), 1996.

(French, 1996) French, C.; Acarnley, P. Direct torque control of permanent magnet drives. IEEE Transaction on Industry Applications, 32(5), 1996. 
(Grenier et al., 1997) Grenier, D., Dessaint, L.-A., Akhrif, O., Bonnassieux, Y., e Pioufle, B. L. Experimental Nonlinear Torque Control of a Permanent-Magnet Synchronous Motor Using Saliency. IEEE Transactions on Industrial Electronics, 44(5), 1997.

(Grenier \& Louis, 1995) Grenier, D. e Louis, J. P. Modeling for control of non-sinewave permanent-magnet synchronous drives by extending Park's transformation. Mathematics and Computers in Simulation, (38), 1995.

(Holtz \& Springob, 1996) Holtz, J. e Springob, L. Identification and compensation of torque ripple in high precision permanent magnet motor drives. IEEE Transaction on Industrial Electronics, 43(2), 1996.

(Jahns, 1996) Jahns, T. M. Pulsating Torque Minimization Techniques for PermanentMagnet AC Motor Drives - a Review. IEEE Transactions on Industrial Electronics, 43(2), 1996.

(Le-Huy et al., 1986) Le-Huy, H., Perret, R., e Feuillet, R. Minimization of Torque Ripple in Brushless DC Motor Drive. IEEE Transactions on Industry Applications, 22, 1986 .

(Miller, 1993) Miller, T. J. E. Bushless Permanent-Magnet and Reluctance Motor Drives. Claredon Press, Oxford, 1993.

(Monteiro, 1997) Monteiro, J. R. B. d. A. Estratégias de Acionamento e Controle em Máquinas CA de Ímã Permanente com Fluxo não Senoidal. São Carlos, 1997. 117p, p. Dissertação (Mestrado) - , Escola de Engenharia de São Carlos, Universidade de São Paulo.

(Morimoto et al., 1994) Morimoto, S., Sanada, M., e Takeda, Y. Wide-Speed Operation of Interior Permanent Magnet Synchronous Motors with High-Performance Current Regulator. IEEE Transactions on Industry Applications, 30(4), 1994.

(Nasar et al., 1993) Nasar, S. A., Boldea, I., e Unnewehr, L. E. Permanent magnet, relutance, and self synchronous motors. CRC Press, Boca Raton, 1993.

(Oliveira Jr., 1990) Oliveira Jr., N. Acionamentos de avanço para máquinas-ferramenta e robôs. comparação entre as técnicas CA/CC. Revista Siemens, 1990. 
(Park et al., 2000) Park, S. J., Park, H. W., Lee, M. H., e Harashima, F. A New Approach for Minimum-Torque-Ripple Maximum-Efficiency Control of BLDC Motor. IEEE Transactions on Industrial Electronics, 47(1), 2000.

(Petrović et al., 2000) Petrović, V., Ortega, R., Stanković, A. M., e Tadmor, G. Design and Implementation of an Adaptative Controler for Torque Ripple Minimization in PM Synchronous Motors. IEEE Transactions on Power Electronics, 15(5), 2000.

(Rahman et al., 1998) Rahman, M. F., Zhong, L., e Lim, K. W. A Direct Torque-Controlled Interior Permanent Magnet Synchronous Motor Drive Incorporating Field Weakening. IEEE Transaction on Industry Applications, 34(6), 1998.

(Sebastian \& Gangla, 1996) Sebastian, T. e Gangla, V. Analysis of induced EMF waveforms and torque ripple in a brushless permanent magnet machine. IEEE Transaction on Industry Applications, 32(1), 1996.

(Takahashi et al., 1994) Takahashi, I., Koganezawa, T., Su, G., e Ohyama, K. A Super High Speed PM Motor Drive System by a Quasi-Current Source Inverter. IEEE Transaction on Industry Applications, 30(3), 1994.

(Trzynadlowski et al., 1997) Trzynadlowski, A., Kirlin, R. L., e Legowski, S. F. Space Vector PWM Technique with Minimum Switching Losses and a Variable Pulse Rate. IEEE Transactions on Industrial Electronics, 44(2), 1997. 